\title{
Catalytic Asymmetric Cyanosilylation of Ketones with Chiral Lewis Base
}

\author{
Shi-Kai Tian, Ran Hong and Li Deng* \\ Department of Chemistry, Brandeis University \\ Waltham, Massachusetts 02454-9110
}

\section{Supporting Information}

General Information. ${ }^{1} \mathrm{H}$ and ${ }^{13} \mathrm{C}$ NMR spectra were recorded on a Varian instrument (400 MHz and $100 \mathrm{MHz}$, respectively) and internally referenced to $\mathrm{SiMe}_{4}$ signal. Exact mass spectra for all new compounds were recorded on a VG 7070 high resolution mass spectrometer. Infrared spectra were recorded on a Perkin Elmer FT-IR Spectrometer. Specific rotations were measured on a Jasco Digital Polarimeter.

Analytical gas-liquid chromatography (GC) was performed on a Hewlett-Packard 6890 Series instrument equipped with a split mode capillary injection system, a flame ionization detector, using a HP-5 GC column or a GC column with chiral stationary phase [Gamma cyclodextrin Trifluoroacetyl $(30 \mathrm{~m} \times 0.25 \mathrm{~mm})]$. All the GC analyses of chiral silylcyanohydrins were carried out with both a crude reaction mixture and a sample purified by silica gel chromatography. High pressure liquid chromatography (HPLC) analyses were performed on a Hewlett-Packard 1100 Series instrument equipped with an isostatic pump, using a Daicel Chiralpak AS (250 x $4.6 \mathrm{~mm}), \mathrm{AD}(250 \times 4.6 \mathrm{~mm})$, OD $(250 \times 4.6 \mathrm{~mm})$, OJ $(250 \times 4.6 \mathrm{~mm})$, or/and Hypersil SI $(200 \times 4.6 \mathrm{~mm})$ Column. UV detection was monitored at $254 \mathrm{~nm}$ or $220 \mathrm{~nm}$.

Ketones (4a-d, 7a), trimethylsilyl cyanide (TMSCN) were purchased from Aldrich and used without further purification. Ketone $\mathbf{7 j}$ was prepared according to a literature procedure. ${ }^{1}$ The yields of the acetal ketone were not optimized yet. The other ketones in Table $1 \& 2$ were prepared as described below. Chloroform was distilled over anhydrous $\mathrm{K}_{2} \mathrm{CO}_{3}$ before use, and commercial ethyl acetate (Aldrich) was used directly without further purification.

Catalyst (DHQ) ${ }_{2}-A Q N$ purification: In most cases, commercially available modified cinchona alkaloids give reproducible results for the asymmetric cyanosilylation. Occasionally, modified cinchona alkaloids purchased from Aldrich Company did not give reproducible results for the asymmetric cyanosilylation. After purification following the extractive procedure described below, the modified cinchona alkaloids give reproducible results.

Commercial modified cinchona alkaloids $(100 \mathrm{mg})$ was dissolved in aqueous $\mathrm{HCl}$ $(0.2 \mathrm{M}, 30 \mathrm{~mL})$ by shaking the mixture at room temperature for $30 \mathrm{~min}$. The resulting yellow solution was extracted with ether $(3 \times 15 \mathrm{~mL})$. The aqueous solution was basified with $\mathrm{K}_{2} \mathrm{CO}_{3}(20 \mathrm{wt} \%$ aq.) to adjust the $\mathrm{pH}$ value of the mixture to 9-11. The resulting mixture was extracted with ether $(3 \times 25 \mathrm{~mL})$, the combined ether solution was washed with distilled water $(3 \times 15 \mathrm{~mL})$ and brine $(3 \times 10 \mathrm{~mL})$, dried over anhydrous sodium

${ }^{1}$ Gupta, S. K. J. Org. Chem. 1976, 41, 2642. 
sulfate, filtered and concentrated under vacuum. The yield of the resulting orange solid was obtained in greater than $98 \%$ and was applied in the asymmetric cyanosilylation to give reproducible results.

\section{General procedure for the DABCO-catalyzed cyanosilylation of ketones}

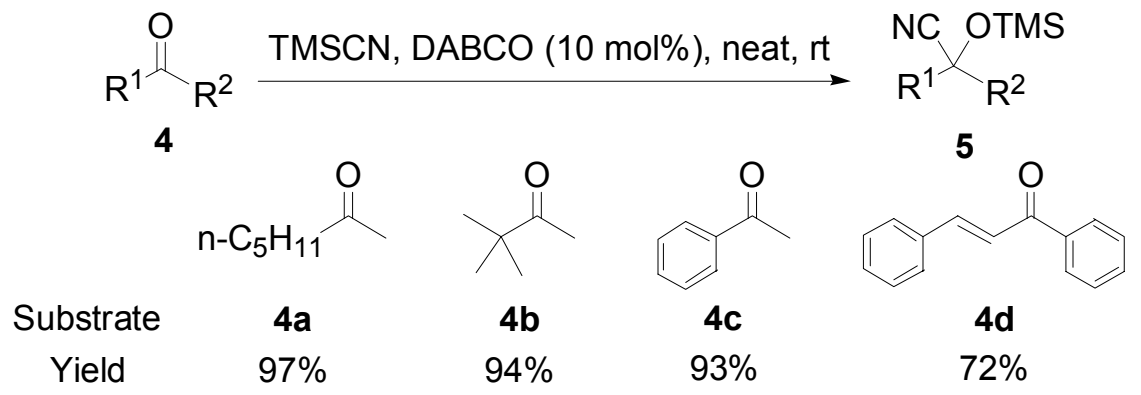

To a mixture of ketone $(0.20 \mathrm{mmol})$ and trimethylsilyl cyanide $(0.60 \mathrm{mmol})$ at room temperature (cooled with water bath) was slowly added DABCO (10 mol\%). The mixture was stirred for 6-24 $\mathrm{h}$ and then purified by silica gel column chromatography (acetone:hexanes/1:100) to give the known silylcyanohydrin (5a-d). ${ }^{2}$

\section{Preparation of the $\alpha, \alpha$-dialkoxymethyl phenyl ketones (6b-c)}

1)

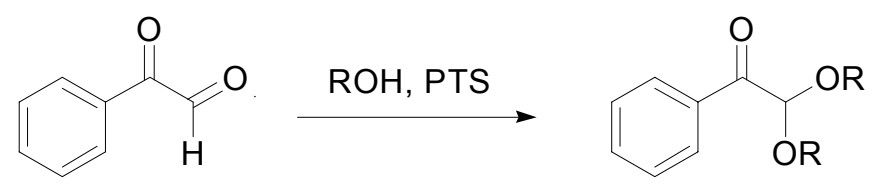

To a suspension of phenylglyoxal monohydrate $(761 \mathrm{mg}, 5.00 \mathrm{mmol})$ in toluene or benzene $(20 \mathrm{~mL})$ was added alcohol $(50.0 \mathrm{mmol})$ and PTS $(86 \mathrm{mg}, 0.50 \mathrm{mmol})$. The resulting mixture was heated at reflux for $2 \mathrm{~h}$, then cooled to room temperature and then concentrated. The residue was purified by silica gel column chromatography (acetone:hexanes/1:100) to give the desired product.

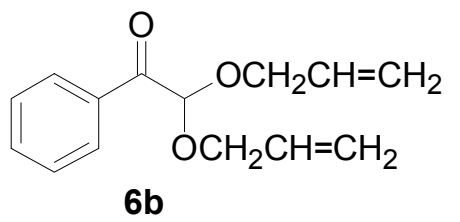

The mixture of phenylglyoxal monohydrate and allyl alcohol was refluxed in benzene for $2 \mathrm{~h}$. The product was obtained as a colorless oil in $55 \%$ yield. ${ }^{1} \mathrm{H}$ NMR $\left(400 \mathrm{MHz}, \mathrm{CDCl}_{3}\right) \delta$ $4.18(\mathrm{dd}, J=12.8,6.0 \mathrm{~Hz}, 2 \mathrm{H}), 4.24(\mathrm{dd}, J=12.8,5.2 \mathrm{~Hz}$, 2H), $5.21(\mathrm{~d}, J=10.4 \mathrm{~Hz}, 2 \mathrm{H}), 5.31(\mathrm{~d}, J=17.6 \mathrm{~Hz}, 2 \mathrm{H})$, $5.38(\mathrm{~s}, 1 \mathrm{H}), 5.87-5.98(\mathrm{~m}, 2 \mathrm{H}), 7.46(\mathrm{t}, J=7.6 \mathrm{~Hz}, 2 \mathrm{H}), 7.57(\mathrm{t}, J=7.4 \mathrm{~Hz}, 1 \mathrm{H}), 8.15(\mathrm{~d}$, $J=7.6 \mathrm{~Hz}, 2 \mathrm{H}) ;{ }^{13} \mathrm{C} \mathrm{NMR}\left(100 \mathrm{MHz}, \mathrm{CDCl}_{3}\right) \delta 68.53,101.19,116.81,118.15,128.58$,

\footnotetext{
${ }^{2}$ Selected references containing physical data, see: for 5a and 5c, Sigeru, T.; Tsutomu, I.; Sadahito, T.; Hirofumi, H.; Hideki, K.; Kenji, U. Bull. Chem. Soc. Jpn. 1987, 60, 2173.; for 5b, Billera, C. F.; Dunn, T. B.; Barry, D. A.; Engel, P. S. J. Org. Chem. 1998, 63, 9763.; for 5d, Choudary, B. M.; Narender, N.; Bhuma, V. Synth. Commun. 1995, 25, 2829.
} 
128.71, 129.94, 133.75, 193.68; IR (neat) $v$ 3071, 1688, 1600, 1584, $1453 \mathrm{~cm}^{-1}$; HRMS (EI) Calcd for $\mathrm{C}_{14} \mathrm{H}_{17} \mathrm{O}_{3}\left(\mathrm{MH}^{+}\right)$233.1178, found 233.1140.

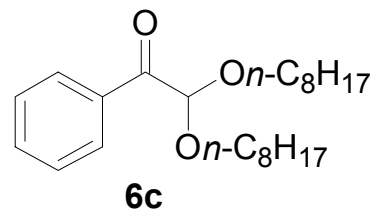

The mixture of phenylglyoxal monohydrate and $n$-octyl alcohol was refluxed in toluene for $2 \mathrm{~h}$. The product was obtained as a colorless oil in $100 \%$ yield. ${ }^{1} \mathrm{H}$ NMR $\left(400 \mathrm{MHz}, \mathrm{CDCl}_{3}\right) \delta 0.87$ $(\mathrm{t}, J=7.2 \mathrm{~Hz}, 6 \mathrm{H}), 1.18-1.29(\mathrm{~m}, 20 \mathrm{H}), 1.55-1.65(\mathrm{~m}, 4 \mathrm{H}), 3.53-$ $3.60(\mathrm{~m}, 2 \mathrm{H}), 3.65-3.72(\mathrm{~m}, 2 \mathrm{H}), 5.23(\mathrm{~s}, 1 \mathrm{H}), 7.42-7.47(\mathrm{~m}, 2 \mathrm{H})$, 7.51-7.57 (m, $1 \mathrm{H}), 8.16(\mathrm{~d}, J=7.2 \mathrm{~Hz}, 2 \mathrm{H}) ;{ }^{13} \mathrm{C} \mathrm{NMR}\left(100 \mathrm{MHz}, \mathrm{CDCl}_{3}\right) \delta 14.29,22.85$, 26.23, 29.42, 29.47, 29.84, 31.99, 68.05, 103.25, 128.49, 128.69, 130.13, 133.60, 194.39; IR (neat) v 2923, 1691, 1599, $1467 \mathrm{~cm}^{-1}$; HRMS (EI) Calcd for $\mathrm{C}_{24} \mathrm{H}_{41} \mathrm{O}_{3}\left(\mathrm{MH}^{+}\right)$ 377.3056, found 377.3031 .

2)

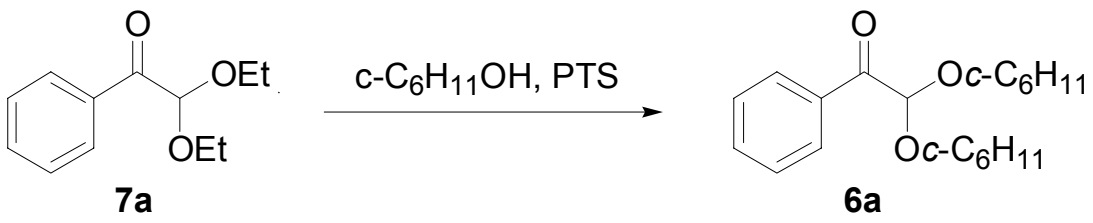

To a solution of 2,2-diethoxyacetophenone (7a) $(1.04 \mathrm{~g}, 5.0 \mathrm{mmol})$ and cyclohexanol $(10.0 \mathrm{~g}, 100 \mathrm{mmol})$ in toluene $(30 \mathrm{~mL})$ was added PTS $(86 \mathrm{mg}, 0.50$ $\mathrm{mmol}$ ). The mixture was refluxed for $2 \mathrm{~h}$. The solvent was removed by distillation under normal pressure and the remaining cyclohexanol was removed under reduced pressure. The residue was purified by silica gel column chromatography (acetone:hexanes/1:100) to give the product (6a) as a colorless oil $(1.52 \mathrm{~g}, 96 \%) .{ }^{1} \mathrm{H}$ NMR $\left(400 \mathrm{MHz}, \mathrm{CDCl}_{3}\right) \delta$ $1.13-1.38(\mathrm{~m}, 8 \mathrm{H}), 1.39-1.54(\mathrm{~m}, 4 \mathrm{H}), 1.59-1.83(\mathrm{~m}, 6 \mathrm{H}), 1.87-1.97(\mathrm{~m}, 2 \mathrm{H}), 3.59-3.67$ $9 \mathrm{~m}, 2 \mathrm{H}), 5.26(\mathrm{~s}, 1 \mathrm{H}), 7.39-7.46(\mathrm{~m}, 2 \mathrm{H}), 7.54(\mathrm{t}, J=7.4 \mathrm{~Hz}, 1 \mathrm{H}), 8.23(\mathrm{~d}, J=7.6 \mathrm{~Hz}$, $2 \mathrm{H}) ;{ }^{13} \mathrm{C}$ NMR $\left(100 \mathrm{MHz}, \mathrm{CDCl}_{3}\right) \delta 24.05,24.26,25.72,32.52,33.20,76.40,102.06$, 128.26, 130.50, 133.31, 133.74, 195.36; IR (neat) v 2933, 1689, 1598, 1580, $1449 \mathrm{~cm}^{-1}$; HRMS (EI) Calcd for $\mathrm{C}_{20} \mathrm{H}_{29} \mathrm{O}_{3}\left(\mathrm{MH}^{+}\right)$317.2117, found 317.2098.

Preparation of the $\alpha, \alpha$-dialkoxymethyl aryl, alkenyl, alkynyl and alkyl ketones $7 \mathrm{~b}-\mathbf{i}$, $7 \mathrm{k}-\mathrm{m}$.

1) Prepared from arylglyoxal monohydrate<smiles>O=CC(=O)Br</smiles><smiles>CC1C=CC=[Pb]C1</smiles><smiles>CCOC(OCC)C(=O)[Al]</smiles>

To a suspension of arylglyoxal monohydrate $(5.00 \mathrm{mmol})$ and PTS $(43 \mathrm{mg}, 0.25$ $\mathrm{mmol})$ in dichloromethane $(20 \mathrm{~mL})$ at room temperature was added dropwise triehyl orthoformate $(10.0 \mathrm{mmol})$. The mixture was stirred for $30 \mathrm{~min}$. Triethylamine $(0.20 \mathrm{~mL})$ was added and the mixture was concentrated. The residue was purified by silica gel 
column chromatography (acetone:hexanes/1:100) to give an $\alpha, \alpha$-diethoxymethyl aryl ketone.<smiles>CCOC(OCC)C(=O)c1ccc(OC)cc1</smiles>

Ketone $7 \mathbf{b}$ was obtained as a colorless oil in $95 \%$ yield. ${ }^{1} \mathrm{H}$ NMR $\left(400 \mathrm{MHz}, \mathrm{CDCl}_{3}\right) \delta 1.25(\mathrm{t}, J=7.0 \mathrm{~Hz}, 6 \mathrm{H}), 3.60-3.72(\mathrm{~m}, 2 \mathrm{H})$, 3.73-3.80 (m, 2H), $3.86(\mathrm{~s}, 3 \mathrm{H}), 5.24(\mathrm{~s}, 1 \mathrm{H}), 6.93(\mathrm{~d}, J=8.8 \mathrm{~Hz}$, $2 \mathrm{H}), 8.16(\mathrm{~d}, J=8.8 \mathrm{~Hz}, 2 \mathrm{H}) ;{ }^{13} \mathrm{C} \mathrm{NMR}\left(100 \mathrm{MHz}, \mathrm{CDCl}_{3}\right) \delta$ $15.35,55.56,63.23,102.74,113.70,126.85,132.28,163.91$, 192.79; IR (neat) $v 2977,1683,1601,1574,1511,1460,1422 \mathrm{~cm}^{-1}$; HRMS (EI) Calcd for $\mathrm{C}_{13} \mathrm{H}_{18} \mathrm{O}_{4}\left(\mathrm{M}^{+}\right)$238.1205, found 238.1179.<smiles>CCOC(OCC)C(=O)c1ccc(Cl)cc1</smiles>

Ketone 7c was obtained as a colorless oil in $96 \%$ yield. ${ }^{3}{ }^{1} \mathrm{H}$ NMR $\left(400 \mathrm{MHz}, \mathrm{CDCl}_{3}\right) \delta 1.25(\mathrm{t}, J=7.0 \mathrm{~Hz}, 6 \mathrm{H}), 3.60-3.68(\mathrm{~m}, 2 \mathrm{H})$, $3.75-3.82(\mathrm{~m}, 2 \mathrm{H}), 5.18(\mathrm{~s}, 1 \mathrm{H}), 7.42(\mathrm{~d}, J=8.8 \mathrm{~Hz}, 2 \mathrm{H}), 8.13(\mathrm{~d}, J$ $=8.8 \mathrm{~Hz}, 2 \mathrm{H}) ;{ }^{13} \mathrm{C} \mathrm{NMR}\left(100 \mathrm{MHz}, \mathrm{CDCl}_{3}\right) \delta 15.34,63.70$, $103.26,128.80,131.49,132.02,140.04,193.16$.

2) Prepared from $\alpha, \alpha$-dipropoxyacetone $(7 \mathbf{j})^{1}$

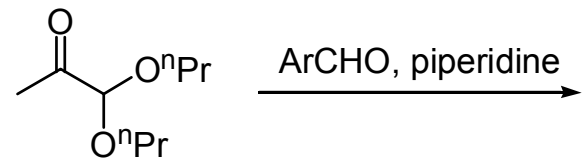

$7 \mathbf{j}$<smiles>CCCOC(OCC)C(=O)C=C[Al]</smiles>

7d-g

To a mixture of $\alpha, \alpha$-dipropoxyacetone (7j) (348 $\mathrm{mg}, 2.0 \mathrm{mmol})$ and aryl aldehyde $(2.5 \mathrm{mmol})$ was slowly added piperidine $(2.00 \mathrm{mmol})$. The mixture was heated at $100{ }^{\circ} \mathrm{C}$ (the temperature of oil bath) until $\alpha, \alpha$-dipropoxyacetone was consumed completely (monitored with GC). The mixture was cooled to room temperature and purified by silica gel column chromatography (acetone:hexanes/1:100) to give an alkenyl ketone.<smiles>CCCOC(OCC)C(=O)/C=C/c1ccccc1</smiles>

The reaction mixture was heated for $4 \mathrm{~h}$ in the presence of 10 mol\% of benzoic acid and the product was obtained as a colorless oil in $70 \%$ yield. ${ }^{1} \mathrm{H}$ NMR $\left(400 \mathrm{MHz}, \mathrm{CDCl}_{3}\right) \delta 0.96(\mathrm{t}, J=7.2$ $\mathrm{Hz}, 6 \mathrm{H}), 1.62-1.71(\mathrm{~m}, 4 \mathrm{H}), 3.49-3.55(\mathrm{~m}, 2 \mathrm{H}), 3.61-3.68(\mathrm{~m}$, $2 \mathrm{H}), 4.81(\mathrm{~s}, 1 \mathrm{H}), 7.12(\mathrm{~d}, J=15.6 \mathrm{~Hz}, 1 \mathrm{H}), 7.38-7.43(\mathrm{~m}, 3 \mathrm{H})$, 7.56-7.62 (m, 2H), $7.80(\mathrm{~d}, J=15.6 \mathrm{~Hz}, 1 \mathrm{H}) ;{ }^{13} \mathrm{C} \mathrm{NMR}\left(100 \mathrm{MHz}, \mathrm{CDCl}_{3}\right) \delta 10.72$, 23.05, 69.37, 102.95, 120.94, 128.72, 129.01, 130.81, 134.77, 144.86, 194.46; IR (neat) $v$ 3060, 2964, 1698, 1611, 1576, 1494, $1450 \mathrm{~cm}^{-1}$; HRMS (EI) Calcd for $\mathrm{C}_{16} \mathrm{H}_{23} \mathrm{O}_{3}\left(\mathrm{MH}^{+}\right)$ 263.1647, found 263.1688 .

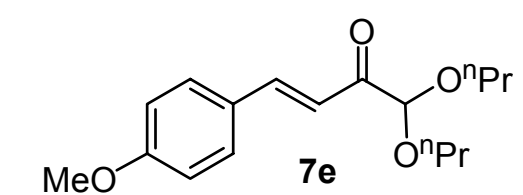

${ }^{3}$ Papillon-Jegou, D.; Bariou, B.; Soyer, N.; Kerfanto, M. Bull. Soc. Chim. Fr. 1977, 977.
The reaction mixture was heated for $7 \mathrm{~h}$ and the product was obtained as a colorless oil in $54 \%$ yield. ${ }^{1} \mathrm{H}$ NMR $\left(400 \mathrm{MHz}, \mathrm{CDCl}_{3}\right) \delta 0.96(\mathrm{t}, J=7.6 \mathrm{~Hz}, 6 \mathrm{H}), 1.62-1.72$ $(\mathrm{m}, 4 \mathrm{H}), 3.52(\mathrm{dt}, J=9.6,6.4 \mathrm{~Hz}, 2 \mathrm{H}), 3.64(\mathrm{dt}, J=9.6$, 
$6.4 \mathrm{~Hz}, 2 \mathrm{H}), 3.84(\mathrm{~s}, 3 \mathrm{H}), 4.80(\mathrm{~s}, 1 \mathrm{H}), 6.91(\mathrm{~d}, J=8.4 \mathrm{~Hz}, 2 \mathrm{H}), 6.99(\mathrm{~d}, J=16.0 \mathrm{~Hz}$, $1 \mathrm{H}), 7.55(\mathrm{~d}, J=8.4 \mathrm{~Hz}, 2 \mathrm{H}), 7.77(\mathrm{~d}, J=16.0 \mathrm{~Hz}, 1 \mathrm{H}) ;{ }^{13} \mathrm{C} \mathrm{NMR}\left(100 \mathrm{MHz}, \mathrm{CDCl}_{3}\right) \delta$ $10.74,23.07,55.52,69.32,102.99,114.49,118.67,127.55,130.54,144.71,161.93$, 194.46; IR (neat) $v$ 2964, 1692, 1597, 1572, 1512, 1463, $1423 \mathrm{~cm}^{-1}$; HRMS $\left(\mathrm{CH}_{4} / \mathrm{CI}\right)$ Calcd for $\mathrm{C}_{17} \mathrm{H}_{25} \mathrm{O}_{4}\left(\mathrm{MH}^{+}\right)$293.1753, found 293.1758.

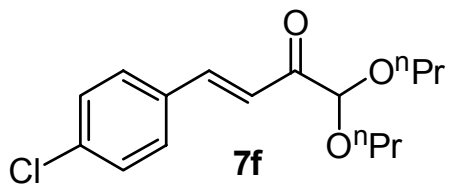

The reaction mixture was heated for $5 \mathrm{~h}$ and the product was obtained as a colorless oil in 52\% yield. ${ }^{1} \mathrm{H}$ NMR $(400 \mathrm{MHz}$, $\left.\mathrm{CDCl}_{3}\right) \delta 0.96(\mathrm{t}, J=7.2 \mathrm{~Hz}, 6 \mathrm{H}), 1.62-1.72(\mathrm{~m}, 4 \mathrm{H}), 3.52$ $(\mathrm{dt}, J=9.6,6.4 \mathrm{~Hz}, 2 \mathrm{H}), 3.64(\mathrm{dt}, J=9.6,6.4 \mathrm{~Hz}, 2 \mathrm{H}), 4.78$ (s, 1H), 7.08 (d, $J=16.0 \mathrm{~Hz}, 1 \mathrm{H}), 7.37$ (d, $J=8.0 \mathrm{~Hz}, 2 \mathrm{H}), 7.53$ (d, $J=8.0 \mathrm{~Hz}, 2 \mathrm{H}), 7.73$ $(\mathrm{d}, J=16.0 \mathrm{~Hz}, 1 \mathrm{H}) ;{ }^{13} \mathrm{C} \mathrm{NMR}\left(100 \mathrm{MHz}, \mathrm{CDCl}_{3}\right) \delta 10.75,23.08,69.56,103.07,121.36$, 129.34, 129.90, 133.32, 136.72, 143.38, 194.29; IR (neat) v 2965, 1700, 1611, 1566, 1491, $1406 \mathrm{~cm}^{-1}$; HRMS $\left(\mathrm{CH}_{4} / \mathrm{CI}\right)$ Calcd for $\mathrm{C}_{16} \mathrm{H}_{22} \mathrm{O}_{3} \mathrm{Cl}\left(\mathrm{MH}^{+}\right)$297.1257, found 297.1254.<smiles>CCCOC(OCCC)C(=O)/C=C/c1cccnc1</smiles>

The reaction mixture was heated for $4 \mathrm{~h}$ and the product was obtained as a colorless oil in $35 \%$ yield. ${ }^{1} \mathrm{H}$ NMR $(400 \mathrm{MHz}$, $\left.\mathrm{CDCl}_{3}\right) \delta 0.96(\mathrm{t}, J=7.2 \mathrm{~Hz}, 6 \mathrm{H}), 1.63-1.72(\mathrm{~m}, 4 \mathrm{H}), 3.52(\mathrm{dt}, J$ $=9.2,6.4 \mathrm{~Hz}, 2 \mathrm{H}), 3.66(\mathrm{dt}, J=9.2,6.4 \mathrm{~Hz}, 2 \mathrm{H}), 4.79(\mathrm{~s}, 1 \mathrm{H})$, $7.18(\mathrm{~d}, J=15.8 \mathrm{~Hz}, 1 \mathrm{H}), 7.34(\mathrm{dd}, J=8.0,4.8 \mathrm{~Hz}, 1 \mathrm{H}), 7.78(\mathrm{~d}$, $J=15.8 \mathrm{~Hz}, 1 \mathrm{H}), 7.92(\mathrm{~d}, J=8.0 \mathrm{~Hz}, 1 \mathrm{H}), 8.61-8.63(\mathrm{~m}, 1 \mathrm{H}), 8.80-8.81(\mathrm{~m}, 1 \mathrm{H}) ;{ }^{13} \mathrm{C}$ NMR $\left(100 \mathrm{MHz}, \mathrm{CDCl}_{3}\right) \delta 10.74,23.07,69.69,103.09,122.77,123.91,130.61,134.70$, 141.06, 150.47, 151.43, 193.96; IR (neat) v 2965, 1701, 1614, 1586, 1476, $1415 \mathrm{~cm}^{-1}$; HRMS $\left(\mathrm{CH}_{4} / \mathrm{CI}\right)$ Calcd for $\mathrm{C}_{15} \mathrm{H}_{22} \mathrm{O}_{3} \mathrm{~N}\left(\mathrm{MH}^{+}\right)$264.1600, found 264.1597.

3) Prepared from diethoxyacetonitrile

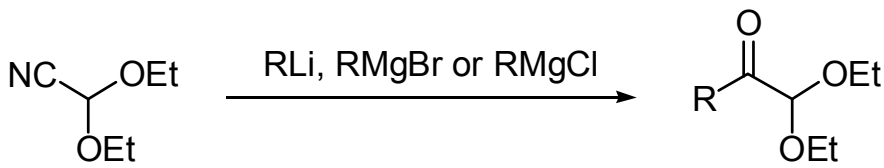

To a solution of diethoxyacetonitrile $(646 \mathrm{mg}, 5.0 \mathrm{mmol})$ in ether $(20 \mathrm{~mL})$ at room temperature (cooled with water bath) was added dropwise a solution of RLi, $\mathrm{RMgBr}$ or $\mathrm{RMgCl}$ (1.0-1.5 eq.) in hexane or ether. The mixture was stirred for $0.5-18 \mathrm{~h}$ and then cooled with ice-water bath. Water $(50 \mathrm{~mL})$ was slowly added and the mixture was extracted with ether $(2 \times 20 \mathrm{~mL})$. The combined organic layers were dried over anhydrous magnesium sulfate, and then concentrated. The residue was purified by silica gel column chromatography (acetone:hexanes/1:100) to give an $\alpha, \alpha$-dialkoxymethyl ketone.<smiles>CCOC(=O)C(C#Cc1ccccc1)OCC</smiles>

The reaction mixture of diethoxyacetonitrile and $\mathrm{PhC} \equiv \mathrm{CMgBr}$ [1.0 eq., prepared in situ by adding EtMgBr (3.0 $\mathrm{M}$ in ether) to a solution of phenylacetylene in ether at room temperature under nitrogen] was stirred for $18 \mathrm{~h}$ and the product was obtained as a 
colorless oil in 41\% yield. ${ }^{4}{ }^{1} \mathrm{H}$ NMR $\left(400 \mathrm{MHz}, \mathrm{CDCl}_{3}\right) \delta 1.31(\mathrm{t}, J=7.2 \mathrm{~Hz}, 6 \mathrm{H}), 3.66-$ $3.82(\mathrm{~m}, 4 \mathrm{H}), 4.87(\mathrm{~s}, 1 \mathrm{H}), 7.37-7.42(\mathrm{~m}, 2 \mathrm{H}), 7.46-7.51(\mathrm{~m}, 1 \mathrm{H}), 7.60-7.64(\mathrm{~m}, 2 \mathrm{H}) ;{ }^{13} \mathrm{C}$ NMR $\left(100 \mathrm{MHz}, \mathrm{CDCl}_{3}\right) \delta 15.37,63.23,86.69,94.89,101.82,128.82,131.24,132.14$, $133.59,183.30$.

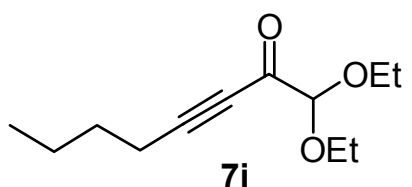

The reaction mixture of diethoxyacetonitrile and $\mathrm{n}-\mathrm{C}_{4} \mathrm{H}_{9} \mathrm{C} \equiv \mathrm{CLi}$ [1.0 eq., prepared in situ by adding BuLi (1.6 M in hexane) to a solution of 1 -hexyne in ether at $0{ }^{\circ} \mathrm{C}$ under nitrogen] was stirred for $0.5 \mathrm{~h}$ and the product was obtained as a colorless oil in $49 \%$ yield. ${ }^{1} \mathrm{H}$ NMR $\left(400 \mathrm{MHz}, \mathrm{CDCl}_{3}\right) \delta 0.93(\mathrm{t}, J=7.0 \mathrm{~Hz}, 3 \mathrm{H})$, $1.27(\mathrm{t}, J=7.0 \mathrm{~Hz}, 6 \mathrm{H}), 1.41-1.49(\mathrm{~m}, 2 \mathrm{H}), 1.55-1.63(\mathrm{~m}, 2 \mathrm{H}), 2.43(\mathrm{t}, J=7.0 \mathrm{~Hz}, 2 \mathrm{H})$, 3.59-3.67 (m, 2H), 3.68-3.76 (m, 2H), $4.73(\mathrm{~s}, 1 \mathrm{H}) ;{ }^{13} \mathrm{C}$ NMR $\left(100 \mathrm{MHz}, \mathrm{CDCl}_{3}\right) \delta$ $13.61,15.23,19.11,22.03,29.69,63.06,79.28,98.87,101.75,183.16$; IR (neat) $v 2963$, 2211, 1684, $1463 \mathrm{~cm}^{-1}$; HRMS $\left(\mathrm{CH}_{4} / \mathrm{CI}\right)$ Calcd for $\mathrm{C}_{12} \mathrm{H}_{21} \mathrm{O}_{3}\left(\mathrm{MH}^{+}\right)$213.1491, found 213.1495 .

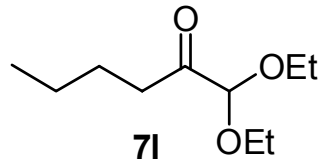

The reaction mixture of diethoxyacetonitrile and BuLi (1.6 M in hexane, 1.0 eq.) was stirred for $0.5 \mathrm{~h}$ and the product was obtained as a colorless oil in 55\% yield. ${ }^{5} \mathrm{H}$ NMR $\left(400 \mathrm{MHz}, \mathrm{CDCl}_{3}\right) \delta 0.91(\mathrm{t}$, $J=7.2 \mathrm{~Hz}, 3 \mathrm{H}), 1.25(\mathrm{t}, J=7.0 \mathrm{~Hz}, 6 \mathrm{H}), 1.28-1.36(\mathrm{~m}, 2 \mathrm{H}), 1.52-$ $1.60(\mathrm{~m}, 2 \mathrm{H}), 2.59(\mathrm{t}, J=7.2 \mathrm{~Hz}, 2 \mathrm{H}), 3.52-3.61(\mathrm{~m}, 2 \mathrm{H}), 3.66-3.74(\mathrm{~m}, 2 \mathrm{H}), 4.56(\mathrm{~s}$, $1 \mathrm{H}) ;{ }^{13} \mathrm{C} \mathrm{NMR}\left(100 \mathrm{MHz}, \mathrm{CDCl}_{3}\right) \delta 14.01,15.30,22.44,25.24,36.65,63.42,102.85$, 206.62 .<smiles>CCOC(OCC)C(=O)C(C)C</smiles>

The reaction mixture of diethoxyacetonitrile and $i-\mathrm{PrMgCl}(2.0 \mathrm{M}$ in ether, 1.5 eq.) was stirred for $0.5 \mathrm{~h}$ and the product was obtained as a colorless oil in 94\% yield. ${ }^{6}{ }^{1} \mathrm{H}$ NMR $\left(400 \mathrm{MHz} \mathrm{CDCl}_{3}\right) \delta 1.10(\mathrm{~d}, J=7.0$ $\mathrm{Hz}, 6 \mathrm{H}), 1.25$ (d, $J=7.2 \mathrm{~Hz}, 6 \mathrm{H}), 3.07$ (sp, $J=7.0 \mathrm{~Hz}, 1 \mathrm{H}), 3.53-3.61$ (m, $2 \mathrm{H}), 3.65-3.73(\mathrm{~m}, 2 \mathrm{H}), 4.70(\mathrm{~s}, 1 \mathrm{H}) ;{ }^{13} \mathrm{C} \mathrm{NMR}\left(100 \mathrm{MHz}, \mathrm{CDCl}_{3}\right) \delta$ $15.25,18.54,35.51,63.13,101.98,209.76$.

4) $7 \mathbf{k}$ was prepared from the alkylation of enamine

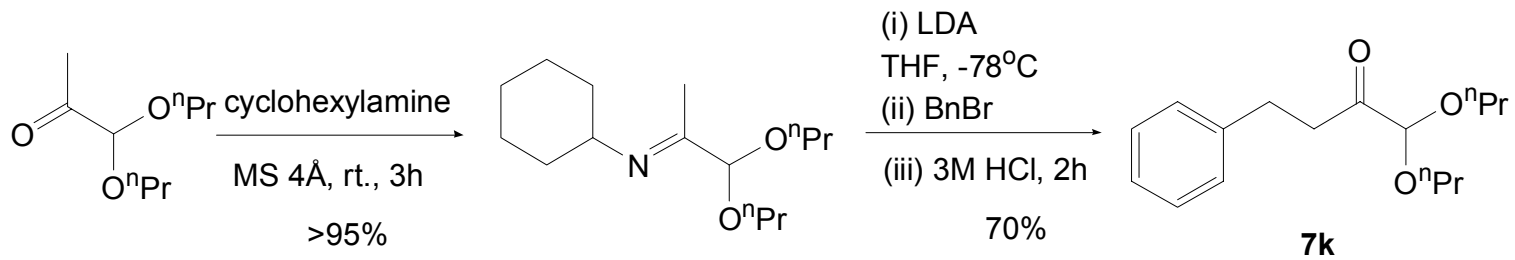

To a flame-dried flask $(250 \mathrm{~mL})$ containing activated MS $4 \AA$ powder was added a solution of $\alpha$, $\alpha$-dipropoxyacetone $(1.74 \mathrm{~g}, 10 \mathrm{mmol})$ in freshly distilled dichloromethane $(12 \mathrm{~mL})$ and cyclohexylamine $\left(1.51 \mathrm{~mL}, 1.31 \mathrm{~g}, 13 \mathrm{mmol}, 1.3\right.$ eq.) under $\mathrm{N}_{2}$ at room

\footnotetext{
${ }^{4}$ Somin, I. N.; Germanguz, E. A. Zh. Org. Khim. 1969, 5, 1780.

${ }^{5}$ Stambouli, A.; Hamedi-Sangsari, F.; Amouroux, R.; Chastrette, F.; Blanc, A.; Mattioda, G. Bull. Soc. Chim. Fr. 1988, 95.

${ }^{6}$ Verhe, R. C.; de Kimpe, N.; de Buyck, L.; Schamp, N. Synthesis, 1982, 667.
} 
temperature. The mixture was stirred at $\mathrm{rt}$ for $3 \mathrm{~h}$. The reaction was monitered by GC. The mixture was filtered through a short pad of Celite ${ }^{\circledR}$ and washed with dichloromethane. The combined filtrates were concentrated and dried in vacuo. The yellow oilish solid was obtained in quantitative yield which was subjected to alkylation without further purification. ${ }^{1} \mathrm{H}$ NMR $\left(400 \mathrm{MHz}, \mathrm{CDCl}_{3}\right): \delta 0.90-1.90(\mathrm{~m}, 23 \mathrm{H}$, alkyl$\mathrm{H})$, 3.31-3.45 (m, $\left.3 \mathrm{H}, \mathrm{OCH}_{2}, \mathrm{CHN}=\right)$, 3.58-3.63 (m, $\left.2 \mathrm{H}, \mathrm{OCH}_{2}\right), 4.54(\mathrm{~s}, 1 \mathrm{H}, \mathrm{CH})$.

To an oven-dried three-necked flask $(250 \mathrm{~mL})$ bearing two addition funnels was added sequentially a solution of LDA (1.9 M in Hex/THF/benzene, $1.65 \mathrm{~mL}, 3.14 \mathrm{mmol}$, 1.1 eq.) and freshly distilled THF $(5 \mathrm{~mL})$ via a syringe under $\mathrm{N}_{2}$ at room temperature. The flask was cooled to $-78^{\circ} \mathrm{C}$ in the dry ice-acetone bath. A solution of the crude imine (700 mg, $2.75 \mathrm{mmol})$ in THF $(5.0 \mathrm{~mL})$ was added dropwise from one funnel in $1 \mathrm{~h}$. The resulting brown mixture was stirred at $-78^{\circ} \mathrm{C}$ for $1 \mathrm{~h}$ and then a solution of benzyl bromide (513 mg, $3.0 \mathrm{mmol}$ ) in THF (5 mL) was added dropwise from other funnel over $30 \mathrm{~min}$. The mixture was stirred at this temperature for $30 \mathrm{~min}$, after the mixture was warmed up to room temperature and stirred at this temperature for an additional $2 \mathrm{~h}$. The reaction was quenched with aqueous $\mathrm{HCl}(3 \mathrm{~N})$ and vigorously stirred at room temperature for $30 \mathrm{~min}$. The $\mathrm{pH}$ value of the mixture was adjusted to 9 using aqueous potassium carbonate $(20 \mathrm{wt} \%)$. Most of the solvent was removed under reduced pressure and the residue was extracted with ether $(3 \times 10 \mathrm{~mL})$. The combined organic layers were washed with brine, dried over anhydrous sodium sulfate ands filtered concentrated. The residue was subjected to silica gel chromatography (Hex:EtOAc $=50: 1)$ to give 3-benzyl$\alpha, \alpha$-dipropoxyacetone (508 mg, 70\% overall yield). ${ }^{1} \mathrm{H} \mathrm{NMR}\left(400 \mathrm{MHz}, \mathrm{CDCl}_{3}\right) \delta 0.92$ $(\mathrm{t}, J=7.2 \mathrm{~Hz}, 6 \mathrm{H}), 1.55-1.65(\mathrm{~m}, 4 \mathrm{H}), 2.90-2.94(\mathrm{~m}, 4 \mathrm{H}), 3.40(\mathrm{dt}, J=9.2,6.4 \mathrm{~Hz}, 2 \mathrm{H})$, $3.56(\mathrm{dt}, J=9.2,6.4 \mathrm{~Hz}, 2 \mathrm{H}), 4.53(\mathrm{~s}, 1 \mathrm{H}), 7.16-7.31(\mathrm{~m}, 5 \mathrm{H}) ;{ }^{13} \mathrm{C} \mathrm{NMR}(100 \mathrm{MHz}$, $\left.\mathrm{CDCl}_{3}\right) \delta 10.72,23.07,29.18,38.62,69.66,103.18,126.20,128.58,141.30,205.64$; IR (neat) $v$ 2965, 1730, 1604, 1496, $1454 \mathrm{~cm}^{-1}$; HRMS (EI) Calcd. for $\mathrm{C}_{16} \mathrm{H}_{24} \mathrm{O}_{3}\left(\mathrm{M}^{+}\right)$ 264.1725 , found 264.1684 .

\section{Structures of commercially available modified cinchona alkaloids}<smiles>[R]C(c1ccnc2ccc(OC)cc12)C1C2CCN1C(CC)C2</smiles>

DHQD-R<smiles>[R]C(c1ccnc2ccc(OC)cc12)[C@@H]1CN2CCC[C@H]1CC2</smiles>

DHQ-R

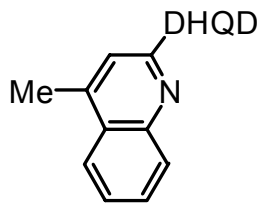

DHQD-MEQ<smiles>Oc1nc(-c2ccccc2)nc(O)c1-c1ccccc1</smiles>

$(\mathrm{DHQD})_{2} \mathrm{PYR}$

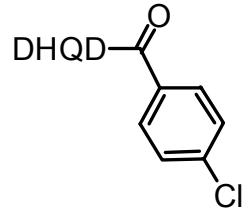

DHQD-CLB<smiles>Oc1nnc(O[OH2+])c2ccccc12</smiles>

$(\mathrm{DHQD})_{2} \mathrm{PHAL}$

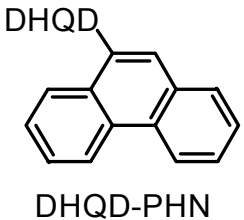<smiles>O=C1c2ccccc2C(=O)c2c(O)ccc(O)c21</smiles>

$(\mathrm{DHQD})_{2} \mathrm{AQN}$ 


\section{General procedure for the preparation of racemic silylcyanohydrins}

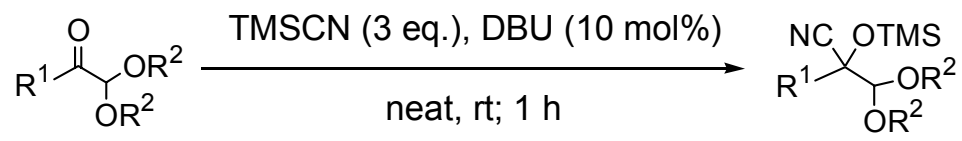

To a mixture of $\alpha, \alpha$-dialkoxyl ketone $(0.20 \mathrm{mmol})$ and trimethylsilyl cyanide $(0.60 \mathrm{mmol})$ at room temperature (cooled with water bath) was slowly added DBU (10 mol\%). The mixture was stirred for $1 \mathrm{~h}$ and then purified by silica gel column chromatography (acetone:hexanes/1:100) to give a racemic silylcyanohydrin in quantitative yield.

General procedure for the modified cinchona alkaloid-catalyzed asymmetric silylcyanation of $\alpha, \alpha$-dialkoxy-substituted ketones

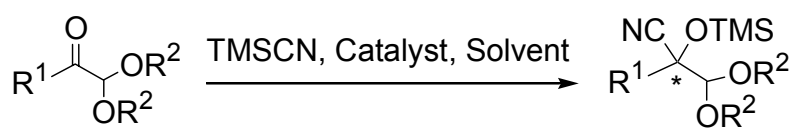

To a solution of $\alpha, \alpha$-dialkoxy ketone $(0.20 \mathrm{mmol})$ and modified cinchona alkaloid $(2-20 \mathrm{~mol} \%$ as indicated in Table $1 \& 2)$ in chloroform or ethyl acetate $(0.20$ $\mathrm{mL})$ at the temperature indicated in Table $1 \& 2$ was added trimethylsilyl cyanide $(0.60$ or $0.40 \mathrm{mmol})$. After being stirred for 7-94 h, methanol $(50 \mu \mathrm{L})$ was added to quench the reaction. The mixture was diluted with hexanes $(5.0 \mathrm{~mL})$ and then washed successively with $0.2 \mathrm{~N}$ aqueous $\mathrm{HCl}$ and water. The organic layer was dried over anhydrous sodium sulfate, and concentrated. The residue was purified by silica gel column chromatography (acetone:hexanes/1:400 to 1:100) to give the chiral silylcyanohydrin.

To the aqueous layer was added potassium carbonate to adjust the $\mathrm{pH}$ value of the solution to $9-11$. The resulting mixture was extracted with ethyl acetate $(10 \mathrm{~mL})$, and the organic layer was washed with brine, dried over anhydrous sodium sulfate, concentrated to afford the recovered catalyst in quantitative yield. The catalyst is shown to be pure by ${ }^{1} \mathrm{H}$ NMR (400 MHz, $\left.\mathrm{CDCl}_{3}\right)$.

\section{1) Products of Table 1 (Entries 1-4)}

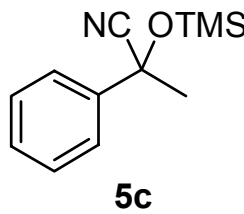

The ee was determined by GC on a chiral support, Gamma cyclodextrin Trifluoroacetyl, $100{ }^{\circ} \mathrm{C}, 4.0 \mathrm{~min}, 0.10{ }^{\circ} \mathrm{C} / \mathrm{min}$ to $102{ }^{\circ} \mathrm{C}$. For a $(\mathrm{DHQ})_{2} \mathrm{AQN}$-catalyzed reaction, $\mathrm{t}_{\mathrm{r}}$ (major) $=18.7$ min, $\mathrm{t}_{\mathrm{r}}($ minor $)=17.9$ min. ${ }^{1} \mathrm{H}$ NMR (400 MHz, $\left.\mathrm{CDCl}_{3}\right) \delta 0.17$ (s, 9H), $1.86(\mathrm{~s}, 3 \mathrm{H}), 7.33-7.42$ $(\mathrm{m}, 3 \mathrm{H}), 7.53-7.56(\mathrm{~m}, 2 \mathrm{H}) ;{ }^{13} \mathrm{C} \mathrm{NMR}\left(100 \mathrm{MHz}, \mathrm{CDCl}_{3}\right) \delta 1.26,33.77$, $94.62,121.19,124.83,128.84,142.21$.

NC OTMS The ee was determined by converting $6 \mathbf{a P}$ to its corresponding $\beta$ Oc- $\mathrm{C}_{6} \mathrm{H}_{11}$ aminoalcohol derivative 6aPD (vide infra). ${ }^{1} \mathrm{H}$ NMR $(400 \mathrm{MHz}$, Oc- $\left.\mathrm{C}_{6} \mathrm{H}_{11} \quad \mathrm{CDCl}_{3}\right) \delta 0.13(\mathrm{~s}, 9 \mathrm{H}), 0.99-1.98(\mathrm{~m}, 20 \mathrm{H}), 3.40-3.46(\mathrm{~m}, 1 \mathrm{H})$, 6aP 
3.60-3.68 (m, 1H), $4.62(\mathrm{~s}, 1 \mathrm{H}), 7.31-7.37(\mathrm{~m}, 3 \mathrm{H}), 7.54-7.58$ 9m, $2 \mathrm{H}) ;{ }^{13} \mathrm{C}$ NMR $(100$ $\left.\mathrm{MHz}, \mathrm{CDCl}_{3}\right) \delta 1.11,23.39,23.73,24.13,24.17,25.65,25.78,31.13,32.30,32.90$, $33.02,75.95,76.83,79.07,102.19,119.72,127.05,127.93,128.78,137.97$; IR (neat) $v$ 2933, 1494, $1450 \mathrm{~cm}^{-1}$; HRMS (EI) Calcd for $\mathrm{C}_{24} \mathrm{H}_{37} \mathrm{NO}_{3} \mathrm{Si}(\mathrm{M}-\mathrm{CN}) 389.2512$, found 389.2501 .<smiles>C=CCOC(O[Na])C(O[Na])(O[Na])c1ccccc1</smiles>

$6 \mathrm{bP}$

The ee was determined by converting $\mathbf{6 b P}$ to its corresponding $\beta$-aminoalcohol derivative $6 \mathbf{b P D}$ (vide infra). ${ }^{1} \mathrm{H}$ NMR $\left(400 \mathrm{MHz}, \mathrm{CDCl}_{3}\right) \delta 0.15(\mathrm{~s}, 9 \mathrm{H}), 3.87$ (dd, $J=$ $12.8,6.0 \mathrm{~Hz}, 1 \mathrm{H}), 4.05(\mathrm{dd}, J=11.8,6.0 \mathrm{~Hz}, 1 \mathrm{H}), 4.22-4.27$ $(\mathrm{m}, 2 \mathrm{H}), 5.04-5.09(\mathrm{~m}, 2 \mathrm{H}), 5.19-5.23(\mathrm{~m}, 1 \mathrm{H}), 5.29-5.34(\mathrm{~m}$, $1 \mathrm{H})$, 5.57-5.68 (m, 1H), 5.87-5.96 (m, $1 \mathrm{H}), 7.35-7.39(\mathrm{~m}, 3 \mathrm{H}), 7.56-7.59(\mathrm{~m}, 2 \mathrm{H}) ;{ }^{13} \mathrm{C}$ NMR $\left(100 \mathrm{MHz}, \mathrm{CDCl}_{3}\right) \delta 1.16,70.54,70.98,78.49,104.73,117.58,117.64,119.35$, 126.90, 128.26, 129.14, 133.67, 134.08, 137.57; IR (neat) $\vee 2956,1648,1492,1450 \mathrm{~cm}^{-1}$; HRMS (EI) Calcd for $\mathrm{C}_{18} \mathrm{H}_{26} \mathrm{NO}_{3} \mathrm{Si}\left(\mathrm{MH}^{+}\right)$332.1682, found 332.1692.

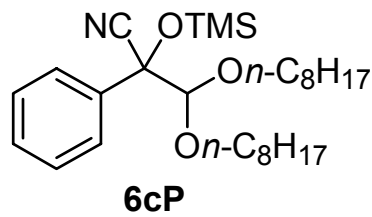

The ee was determined by converting $\mathbf{6 c P}$ to its corresponding $\beta$ aminoalcohol derivative 6cPD (vide infra). ${ }^{1} \mathrm{H}$ NMR $(400 \mathrm{MHz}$, $\left.\mathrm{CDCl}_{3}\right) \delta 0.16(\mathrm{~s}, 9 \mathrm{H}), 0.86-0.91(\mathrm{~m}, 6 \mathrm{H}), 1.08-1.42(\mathrm{~m}, 20 \mathrm{H})$, 1.56-1.66 (m, 4H), 3.38-3.65 (m, 4H), $4.38(\mathrm{~s}, 1 \mathrm{H}), 7.33-7.37(\mathrm{~m}$, $3 \mathrm{H}), 7.54-7.57(\mathrm{~m}, 2 \mathrm{H}) ;{ }^{13} \mathrm{C}$ NMR $\left(100 \mathrm{MHz}, \mathrm{CDCl}_{3}\right) \delta 1.18$, $14.29,22.85,26.00,26.23,29.40,29.58,29.95,30.27,31.98,32.03,70.49,70.73,78.41$, 106.73, 119.55, 126.90, 128.12, 128.93, 137.93; IR (neat) $\vee 2927,1467 \mathrm{~cm}^{-1}$; HRMS (EI) Calcd for $\mathrm{C}_{28} \mathrm{H}_{50} \mathrm{NO}_{3} \mathrm{Si}\left(\mathrm{MH}^{+}\right)$476.3560, found 476.3569.

\section{2) Products of Table 2}<smiles>CCOC(OCC)C(C#N)(OCC)c1ccccc1</smiles>

$(+)-8 \mathbf{a}$

(+)-8a was obtained as a colorless oil in 98\% isolated yield and $90 \%$ ee [determined by converting this compound to its corresponding $\beta$ aminoalcohol derivative 8aD (vide infra)] from a (DHQ) ${ }_{2} \mathrm{AQN}(2$ mol\%)-catalyzed reaction which was run with 3.0 eq. of trimethylsilyl cyanide in chloroform at $-50{ }^{\circ} \mathrm{C}$ for $19 \mathrm{~h} . \quad[\alpha]_{\mathrm{D}}{ }^{25}=15.7$ (c 1.28, $\left.\mathrm{CHCl}_{3}\right) ;{ }^{1} \mathrm{H} \mathrm{NMR}\left(400 \mathrm{MHz}, \mathrm{CDCl}_{3}\right) \delta 0.17(\mathrm{~s}, 9 \mathrm{H}), 1.03(\mathrm{t}, J=7.0 \mathrm{~Hz}, 3 \mathrm{H}), 1.25(\mathrm{t}, J=$ $7.2 \mathrm{~Hz}, 3 \mathrm{H}), 3.24-3.33(\mathrm{~m}, 1 \mathrm{H}), 3.61-3.72(\mathrm{~m}, 2 \mathrm{H}), 3.77-3.85(\mathrm{~m}, 1 \mathrm{H}), 4.38(\mathrm{~s}, 1 \mathrm{H}), 7.34-$ $7.40(\mathrm{~m}, 3 \mathrm{H}), 7.55-7.59(\mathrm{~m}, 2 \mathrm{H}) ;{ }^{13} \mathrm{C}$ NMR $\left(100 \mathrm{MHz}, \mathrm{CDCl}_{3}\right) \delta 1.15,14.99,15.31$, 66.01, 78.28, 106.42, 119.53, 126.86, 128.12, 128.97, 137.74; IR (neat) v 2978, 1602, 1493, $1450 \mathrm{~cm}^{-1}$; HRMS (DCI/NH ${ }_{3}$ ) Calcd for $\mathrm{C}_{16} \mathrm{H}_{29} \mathrm{~N}_{2} \mathrm{O}_{3} \mathrm{Si}\left(\mathrm{MNH}_{4}{ }^{+}\right)$325.1947, found 325.1949 .<smiles>CCOC(OCC)C(C#N)(OCC)c1ccc(OC)cc1</smiles>

$(+)-8 b$

$(+)-8 \mathbf{b}$ was obtained as a colorless oil in $94 \%$ isolated yield and 97\% ee [determined with chiral HPLC, Daicel Chiralpak AD, isopropanol:hexane $/ 0.2: 99.8,0.20 \mathrm{~mL} / \mathrm{min}, \lambda=220 \mathrm{~nm}, \mathrm{t}_{\mathrm{r}}$ $($ major $)=29.9 \mathrm{~min}, \mathrm{t}_{\mathrm{r}}($ minor $\left.)=33.7 \mathrm{~min}\right]$ from a $(\mathrm{DHQ})_{2} \mathrm{AQN}(2$ mol\%)-catalyzed reaction which was run with 3.0 eq. of trimethylsilyl cyanide in chloroform at $-50{ }^{\circ} \mathrm{C}$ for $18 \mathrm{~h}$. $[\alpha]_{\mathrm{D}}{ }^{25}=16.9\left(\mathrm{c} 1.84, \mathrm{CHCl}_{3}\right) ;{ }^{1} \mathrm{H}$ NMR $(400 \mathrm{MHz}$, 
$\left.\mathrm{CDCl}_{3}\right) \delta 0.17(\mathrm{~s}, 9 \mathrm{H}), 1.06(\mathrm{t}, J=7.2 \mathrm{~Hz}, 3 \mathrm{H}), 1.25(\mathrm{t}, J=7.2 \mathrm{~Hz}, 3 \mathrm{H}), 3.28-3.36(\mathrm{~m}$, $1 \mathrm{H}), 3.62-3.72(\mathrm{~m}, 2 \mathrm{H}), 3.76-3.84(\mathrm{~m}, 1 \mathrm{H}), 3.82(\mathrm{~s}, 3 \mathrm{H}), 4.36(\mathrm{~s}, 1 \mathrm{H}), 6.89(\mathrm{~d}, J=8.8 \mathrm{~Hz}$, 2H), $7.48(\mathrm{~d}, J=8.8 \mathrm{~Hz}, 2 \mathrm{H}) ;{ }^{13} \mathrm{C} \mathrm{NMR}\left(100 \mathrm{MHz}, \mathrm{CDCl}_{3}\right) \delta 1.16,15.08,15.29,55.41$, 65.94, 66.06, 77.88, 106.38, 113.42, 119.71, 128.15, 129.68, 160.03; IR (neat) v 2977, 1611, 1512, $1464 \mathrm{~cm}^{-1}$; HRMS (DCI/NH$)$ Calcd for $\mathrm{C}_{17} \mathrm{H}_{31} \mathrm{~N}_{2} \mathrm{O}_{4} \mathrm{Si}\left(\mathrm{MNH}_{4}{ }^{+}\right)$355.2053, found 355.2049 .

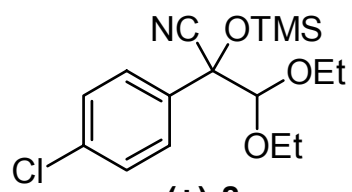

$(+)-8 c$

$(+)-8 c$ was obtained as a colorless oil in $96 \%$ isolated yield and $98 \%$ ee [determined with GC on a chiral support, Gamma cyclodextrin Trifluoroacetyl, $115^{\circ} \mathrm{C}, 4.0 \mathrm{~min}, 0.05^{\circ} \mathrm{C} / \mathrm{min}$ to 122 ${ }^{\circ} \mathrm{C}, \mathrm{t}_{\mathrm{r}}$ (major) $=107.7 \mathrm{~min}, \mathrm{t}_{\mathrm{r}}$ (minor) $\left.=110.4 \mathrm{~min}\right]$ from a (DHQ) ${ }_{2} \mathrm{AQN}(2 \mathrm{~mol} \%$ )-catalyzed reaction which was run with 3.0 eq. of trimethylsilyl cyanide in chloroform at $-50{ }^{\circ} \mathrm{C}$ for $18 \mathrm{~h} .[\alpha]_{\mathrm{D}}{ }^{25}=15.3$ (c 1.82 , $\left.\mathrm{CHCl}_{3}\right) ;{ }^{1} \mathrm{H}$ NMR $\left(400 \mathrm{MHz}, \mathrm{CDCl}_{3}\right) \delta 0.19(\mathrm{~s}, 9 \mathrm{H}), 1.08(\mathrm{t}, J=7.0 \mathrm{~Hz}, 3 \mathrm{H}), 1.25(\mathrm{t}, J=$ $7.0 \mathrm{~Hz}, 3 \mathrm{H}), 3.33-3.41(\mathrm{~m}, 1 \mathrm{H}), 3.64-3.73(\mathrm{~m}, 2 \mathrm{H}), 3.76-3.84(\mathrm{~m}, 1 \mathrm{H}), 4.38(\mathrm{~s}, 1 \mathrm{H}), 7.35$ $(\mathrm{d}, J=8.8 \mathrm{~Hz}, 2 \mathrm{H}), 7.51(\mathrm{~d}, J=8.8 \mathrm{~Hz}, 2 \mathrm{H}) ;{ }^{13} \mathrm{C} \mathrm{NMR}\left(100 \mathrm{MHz}, \mathrm{CDCl}_{3}\right) \delta 1.13,15.07$, $15.25,66.14,66.24,77.65,106.12,119.27,128.29,128.38,134.98,136.18$; IR (neat) $v$ 2978, 1595, 1491, 1446, $1402 \mathrm{~cm}^{-1}$; HRMS (DCI/NH $)_{3}$ Calcd for $\mathrm{C}_{16} \mathrm{H}_{28} \mathrm{~N}_{2} \mathrm{O}_{3} \mathrm{ClSi}$ $\left(\mathrm{MNH}_{4}^{+}\right)$359.1558, found 359.1568.

(-)-8c was obtained as a colorless oil in 95\% isolated yield and 94\% ee from a (DHQD) $)_{2}$ PHAL $(10 \mathrm{~mol} \%)$-catalyzed reaction which was run with 2.0 eq. of trimethylsilyl cyanide in chloroform at $-50{ }^{\circ} \mathrm{C}$ for $40 \mathrm{~h}$.

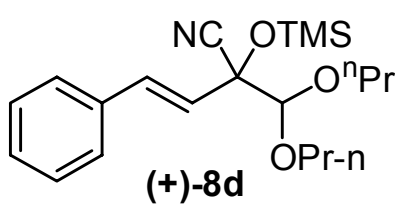

$(+)-8 d$ was obtained as a colorless oil in 93\% isolated yield and 91\% ee [determined with chiral HPLC, Daicel Chiralpak AD, isopropanol:hexane $/ 1: 99,0.10 \mathrm{~mL} / \mathrm{min}, \lambda=254 \mathrm{~nm}, \mathrm{t}_{\mathrm{r}}$ (major) $=$ $37.7 \mathrm{~min}, \mathrm{t}_{\mathrm{r}}($ minor $\left.)=41.1 \mathrm{~min}\right]$ from a $(\mathrm{DHQ})_{2} \mathrm{AQN}(2 \mathrm{~mol} \%)-$ catalyzed reaction which was run with 3.0 eq. of trimethylsilyl cyanide in chloroform at $-50{ }^{\circ} \mathrm{C}$ for $16 \mathrm{~h} .[\alpha]_{\mathrm{D}}{ }^{25}=31.9\left(\mathrm{c} 2.03, \mathrm{CHCl}_{3}\right) ;{ }^{1} \mathrm{H}$ NMR (400 $\left.\mathrm{MHz} \mathrm{CDCl}_{3}\right) \delta 0.24(\mathrm{~s}, 9 \mathrm{H}), 0.94(\mathrm{t}, J=7.2 \mathrm{~Hz}, 3 \mathrm{H}), 0.96(\mathrm{t}, J=7.2 \mathrm{~Hz}, 3 \mathrm{H}), 1.60-1.70$ $(\mathrm{m}, 4 \mathrm{H}), 3.53-3.62(\mathrm{~m}, 2 \mathrm{H}), 3.70-3.77(\mathrm{~m}, 2 \mathrm{H}), 4.45(\mathrm{~s}, 1 \mathrm{H}), 6.25(\mathrm{~d}, J=16.0 \mathrm{~Hz}, 1 \mathrm{H})$, $6.93(\mathrm{~d}, J=16.0 \mathrm{~Hz}, 1 \mathrm{H}), 7.28-7.45(\mathrm{~m}, 5 \mathrm{H}) ;{ }^{13} \mathrm{C} \mathrm{NMR}\left(100 \mathrm{MHz}, \mathrm{CDCl}_{3}\right) \delta 1.59,10.79$, 10.86, 23.14, 23.25, 72.15, 72.49, 94.62, 105.91, 119.01, 124.83, 127.26, 128.72, 128.90, 133.90, 135.69; IR (neat) $v 2962,1450 \mathrm{~cm}^{-1}$; HRMS (DCI/ $\mathrm{NH}_{3}$ ) Calcd for $\mathrm{C}_{20} \mathrm{H}_{35} \mathrm{~N}_{2} \mathrm{O}_{3} \mathrm{Si}$ $\left(\mathrm{MNH}_{4}{ }^{+}\right)$379.2417, found 379.2412.

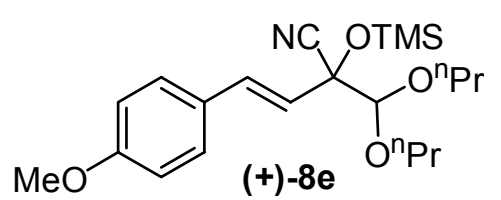

$(+)-8 e$ was obtained as a colorless oil in $92 \%$ isolated yield and $90 \%$ ee [determined with chiral HPLC, Daicel Chiralpak AD, isopropanol:hexane $/ 1: 99,0.20 \mathrm{~mL} / \mathrm{min}, \lambda=$ $254 \mathrm{~nm}, \mathrm{t}_{\mathrm{r}}($ major $)=22.6 \mathrm{~min}, \mathrm{t}_{\mathrm{r}}($ minor $\left.)=26.0 \mathrm{~min}\right]$ from a (DHQ) ${ }_{2} \mathrm{AQN}(2 \mathrm{~mol} \%)$-catalyzed reaction which was run with 3.0 eq. of trimethylsilyl cyanide in chloroform at $-50{ }^{\circ} \mathrm{C}$ for $18 \mathrm{~h}$. $[\alpha]_{\mathrm{D}}{ }^{25}=71.6$ (c 1.52, $\left.\mathrm{CHCl}_{3}\right) ;{ }^{1} \mathrm{H}$ NMR (400 MHz, $\left.\mathrm{CDCl}_{3}\right) \delta 0.24(\mathrm{~s}, 9 \mathrm{H}), 0.94(\mathrm{t}, J=7.6 \mathrm{~Hz}, 3 \mathrm{H}), 0.95$ $(\mathrm{t}, J=7.6 \mathrm{~Hz}, 3 \mathrm{H}), 1.59-1.70(\mathrm{~m}, 4 \mathrm{H}), 3.52-3.62(\mathrm{~m}, 2 \mathrm{H}), 3.71-3.78(\mathrm{~m}, 2 \mathrm{H}), 3.81(\mathrm{~s}$, $3 \mathrm{H}), 4.45(\mathrm{~s}, 1 \mathrm{H}), 6.11(\mathrm{~d}, J=16.0 \mathrm{~Hz}, 1 \mathrm{H}), 6.88(\mathrm{~d}, J=16.0 \mathrm{~Hz}, 1 \mathrm{H}), 6.88(\mathrm{~d}, J=8.8$ 
$\mathrm{Hz}, 2 \mathrm{H}), 7.37(\mathrm{~d}, J=8.8 \mathrm{~Hz}, 2 \mathrm{H}) ;{ }^{13} \mathrm{C} \mathrm{NMR}\left(100 \mathrm{MHz}, \mathrm{CDCl}_{3}\right) \delta 1.56,10.74,10.81$, 23.09, 23.20, 55.46, 72.05, 72.44, 77.05, 105.88, 114.24, 119.08, 122.38, 128.37, 128.50, 133.40, 160.08; IR (neat) v 2963, 1652, 1608, 1577, 1513, $1464 \mathrm{~cm}^{-1}$; HRMS (DCI/NH $\left.\mathrm{NH}_{3}\right)$ Calcd for $\mathrm{C}_{21} \mathrm{H}_{37} \mathrm{~N}_{2} \mathrm{O}_{4} \mathrm{Si}\left(\mathrm{MNH}_{4}^{+}\right)$409.2523, found 409.2522.

(-)-8e was obtained as a colorless oil in 96\% isolated yield and $92 \%$ ee as determined by chiral HPLC from a (DHQD) ${ }_{2}$ PHAL (10 mol\%)-catalyzed reaction which was run with 3.0 eq. of trimethylsilyl cyanide in ethyl acetate at $-30{ }^{\circ} \mathrm{C}$ for $21 \mathrm{~h}$.

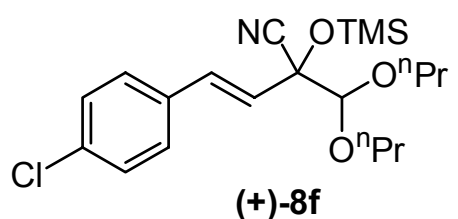

$(+)-8 f$

(+)-8f was obtained as a colorless oil in 95\% isolated yield and $92 \%$ ee [determined with chiral HPLC [Daicel Chiralpak $\mathrm{AD}$, isopropanol:hexane $/ 0.2: 99.8,1.0 \mathrm{~mL} / \mathrm{min}, \lambda=254 \mathrm{~nm}, \mathrm{t}_{\mathrm{r}}$ (major) $=16.8 \mathrm{~min}, \mathrm{t}_{\mathrm{r}}$ (minor) $\left.=20.2 \mathrm{~min}\right]$ from $\mathrm{a}$ (DHQ) ${ }_{2} \mathrm{AQN}(2 \mathrm{~mol} \%)$-catalyzed reaction which was run with 3.0 eq. of trimethylsilyl cyanide in chloroform at $-50{ }^{\circ} \mathrm{C}$ for $18 \mathrm{~h}$. $[\alpha]_{\mathrm{D}}{ }^{25}=47.0$ (c 2.01, $\left.\mathrm{CHCl}_{3}\right) ;{ }^{1} \mathrm{H}$ NMR $\left(400 \mathrm{MHz}, \mathrm{CDCl}_{3}\right) \delta 0.24(\mathrm{~s}, 9 \mathrm{H}), 0.93(\mathrm{t}, J=7.2 \mathrm{~Hz}, 3 \mathrm{H}), 0.95$ $(\mathrm{t}, J=7.2 \mathrm{~Hz}, 3 \mathrm{H}), 1.59-1.70(\mathrm{~m}, 4 \mathrm{H}), 3.54-3.62(\mathrm{~m}, 2 \mathrm{H}), 3.71-3.79(\mathrm{~m}, 2 \mathrm{H}), 4.46(\mathrm{~s}$, $1 \mathrm{H}), 6.23(\mathrm{~d}, J=16.0 \mathrm{~Hz}, 1 \mathrm{H}), 6.88(\mathrm{~d}, J=16.0 \mathrm{~Hz}, 1 \mathrm{H}), 7.30-7.38(\mathrm{~m}, 4 \mathrm{H}) ;{ }^{13} \mathrm{C} \mathrm{NMR}$ $\left(100 \mathrm{MHz}, \mathrm{CDCl}_{3}\right) \delta 1.53,10.74,10.81,23.10,23.23,72.17,72.56,76.78,105.83$, $118.83,125.47,128.42,129.08,132.62,134.15,134.44$; IR (neat) $v 2964,1612,1594$, 1492, 1464, $1406 \mathrm{~cm}^{-1}$; HRMS (DCI/ $\left.\mathrm{NH}_{3}\right)$ Calcd for $\mathrm{C}_{20} \mathrm{H}_{34} \mathrm{~N}_{2} \mathrm{O}_{3} \mathrm{SiCl}\left(\mathrm{MNH}_{4}^{+}\right)$ 413.2027, found 413.2022.

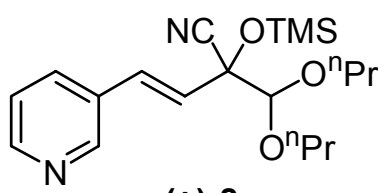

$(+)-8 g$

$(+)-8 g$ was obtained as a colorless oil in 97\% isolated yield and 93\% ee [determined with chiral HPLC, Daicel Chiralpak AD, isopropanol:hexane/1:99, $0.50 \mathrm{~mL} / \mathrm{min}, \lambda=254 \mathrm{~nm}, \mathrm{t}_{\mathrm{r}}$ (major) $=$ $29.2 \mathrm{~min}, \mathrm{t}_{\mathrm{r}}($ minor $\left.)=33.6 \mathrm{~min}\right]$ from a $(\mathrm{DHQ})_{2} \mathrm{AQN}(2 \mathrm{~mol} \%)-$ catalyzed reaction which was run with 3.0 eq. of trimethylsilyl cyanide in chloroform at $-50{ }^{\circ} \mathrm{C}$ for $18 \mathrm{~h}$. $[\alpha]_{\mathrm{D}}{ }^{25}=37.8\left(\mathrm{c} 1.60, \mathrm{CHCl}_{3}\right) ;{ }^{1} \mathrm{H}$ NMR (400 $\left.\mathrm{MHz} \mathrm{CDCl}_{3}\right) \delta 0.25(\mathrm{~s}, 9 \mathrm{H}), 0.94(\mathrm{t}, J=7.2 \mathrm{~Hz}, 3 \mathrm{H}), 0.96(\mathrm{t}, J=7.2 \mathrm{~Hz}, 3 \mathrm{H}), 1.59-1.71$ $(\mathrm{m}, 4 \mathrm{H}), 3.54-3.63(\mathrm{~m}, 2 \mathrm{H}), 3.72-3.79(\mathrm{~m}, 2 \mathrm{H}), 4.48(\mathrm{~s}, 1 \mathrm{H}), 6.34(\mathrm{~d}, J=16.0 \mathrm{~Hz}, 1 \mathrm{H})$, $6.91(\mathrm{~d}, J=16.0 \mathrm{~Hz}, 1 \mathrm{H}), 7.27-7.32(\mathrm{~m}, 1 \mathrm{H}), 7.73-7.77(\mathrm{~m}, 1 \mathrm{H}), 8.52-8.54(\mathrm{~m}, 1 \mathrm{H}), 8.65-$ $8.67(\mathrm{~m}, 1 \mathrm{H}) ;{ }^{13} \mathrm{C} \mathrm{NMR}\left(100 \mathrm{MHz}, \mathrm{CDCl}_{3}\right) \delta 1.54,10.75,10.82,23.09,23.23,72.27$, 72.65, 76.62, 105.81, 118.68, 123.80, 127.24, 130.27, 131.38, 133.77, 148.87, 149.64; IR (neat) $v 2963,1613,1478,1416 \mathrm{~cm}^{-1}$; HRMS $\left(\mathrm{DCI} / \mathrm{NH}_{3}\right)$ Calcd for $\mathrm{C}_{19} \mathrm{H}_{31} \mathrm{~N}_{2} \mathrm{O}_{3} \mathrm{Si}\left(\mathrm{MH}^{+}\right)$ 363.2104, found 363.2097.

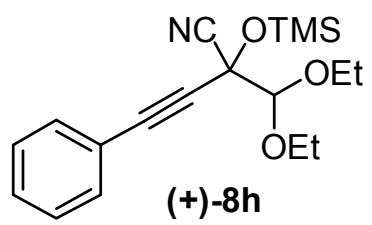

$(+)-8 \mathbf{h}$ was obtained as a colorless oil in $93 \%$ isolated yield and $96 \%$ ee [determined with chiral HPLC, Hypersil SI + Daicel Chiralpak AS, isopropanol:hexane $/ 1: 99,0.20 \mathrm{~mL} / \mathrm{min}, \lambda=254$ $\mathrm{nm}, \mathrm{t}_{\mathrm{r}}$ (major) $=35.3 \mathrm{~min}, \mathrm{t}_{\mathrm{r}}($ minor $\left.)=37.2 \mathrm{~min}\right]$ from a (DHQ) ${ }_{2} \mathrm{AQN}(2 \mathrm{~mol} \%)$-catalyzed reaction which was run with 3.0 eq. of trimethylsilyl cyanide in chloroform at $-50{ }^{\circ} \mathrm{C}$ for $19 \mathrm{~h}$. $[\alpha]_{\mathrm{D}}{ }^{25}=12.9\left(\mathrm{c} 1.26, \mathrm{CHCl}_{3}\right) ;{ }^{1} \mathrm{H} \mathrm{NMR}(400$ $\left.\mathrm{MHz}, \mathrm{CDCl}_{3}\right) \delta 0.34(\mathrm{~s}, 9 \mathrm{H}), 1.26-1.36(\mathrm{~m}, 6 \mathrm{H}), 3.73-3.93(\mathrm{~m}, 4 \mathrm{H}), 4.55(\mathrm{~s}, 1 \mathrm{H}), 7.32-$ $7.42(\mathrm{~m}, 3 \mathrm{H}), 7.46-7.51(\mathrm{~m}, 2 \mathrm{H}) ;{ }^{13} \mathrm{C} \mathrm{NMR}\left(100 \mathrm{MHz}, \mathrm{CDCl}_{3}\right) \delta 1.27,15.37,63.22$, $66.03,66.13,83.38,87.70,105.03,117.51,128.60,129.61,132.06,133.60$; IR (neat) $v$ 
2978, 2204, 1682, 1490, $1444 \mathrm{~cm}^{-1}$; HRMS (EI) Calcd for $\mathrm{C}_{18} \mathrm{H}_{26} \mathrm{NO}_{3} \mathrm{Si}\left(\mathrm{MH}^{+}\right)$332.1682, found 332.1647 .

(-)-8h was obtained as a colorless oil in 96\% isolated yield and $93 \%$ ee as determined by chiral HPLC from a (DHQD) $)_{2}$ PHAL (10 mol\%)-catalyzed reaction which was run with 3.0 eq. of trimethylsilyl cyanide in ethyl acetate at $-30{ }^{\circ} \mathrm{C}$ for $21 \mathrm{~h}$.

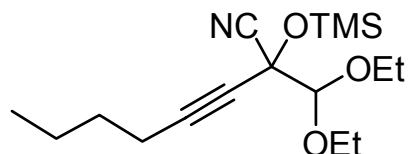

$(+)-8 \mathbf{i}$

$(+)-8 \mathbf{i}$ was obtained as a colorless oil in $94 \%$ isolated yield and $95 \%$ ee [determined by converting this compound to its corresponding $\beta$-aminoalcohol derivative 8iD (vide infra)] from a (DHQ) ${ }_{2} \mathrm{AQN}(2 \mathrm{~mol} \%)$-catalyzed reaction which was run with 3.0 eq. of trimethylsilyl cyanide in chloroform at $-50{ }^{\circ} \mathrm{C}$ for 18 h. $[\alpha]_{\mathrm{D}}{ }^{25}=1.9\left(\mathrm{c} 1.56, \mathrm{CHCl}_{3}\right) ;{ }^{1} \mathrm{H}$ NMR $\left(400 \mathrm{MHz}, \mathrm{CDCl}_{3}\right) \delta 0.29(\mathrm{~s}, 9 \mathrm{H}), 0.92(\mathrm{t}, J=$ $7.0 \mathrm{~Hz}, 3 \mathrm{H}), 1.26(\mathrm{t}, J=7.0 \mathrm{~Hz}, 3 \mathrm{H}), 1.27(\mathrm{t}, J=7.0 \mathrm{~Hz}, 3 \mathrm{H}), 1.38-1.48(\mathrm{~m}, 2 \mathrm{H}), 1.49-$ $1.56(\mathrm{~m}, 2 \mathrm{H}), 2.27(\mathrm{t}, J=7.0 \mathrm{~Hz}, 3 \mathrm{H}), 3.68-3.77(\mathrm{~m}, 2 \mathrm{H}), 3.81-3.89(\mathrm{~m}, 2 \mathrm{H}), 4.42(\mathrm{~s}$, $1 \mathrm{H}) ;{ }^{13} \mathrm{C}$ NMR $\left(100 \mathrm{MHz}, \mathrm{CDCl}_{3}\right) \delta 1.21,13.68,15.28,18.63,22.11,30.07,65.97$, 67.90, 74.61, 89.40, 104.97, 117.88; IR (neat) $v$ 2964, 2360, $1455 \mathrm{~cm}^{-1}$; HRMS (DCI/NH$)$ Calcd for $\mathrm{C}_{16} \mathrm{H}_{33} \mathrm{~N}_{2} \mathrm{O}_{3} \mathrm{Si}\left(\mathrm{MNH}_{4}{ }^{+}\right)$329.2260, found 329.2256.

NC OTMS $\boldsymbol{R}-(-)-8 j$ was obtained as a colorless oil in $97 \%$ isolated yield and $92 \%$ ee $\mathrm{O}^{\mathrm{n}} \operatorname{Pr}$ [determined with $\mathrm{GC}$ on a chiral support, Gamma cyclodextrin ${ }_{\mathrm{O}} \mathrm{Pr}$ Trifluoroacetyl, $95{ }^{\circ} \mathrm{C}, 4.0 \mathrm{~min}, 0.10{ }^{\circ} \mathrm{C} / \mathrm{min}$ to $98{ }^{\circ} \mathrm{C}, \mathrm{t}_{\mathrm{r}}$ (major) $=25.9$ min, $t_{r}($ minor $\left.)=25.2 \mathrm{~min}\right]$ from a $(\mathrm{DHQ})_{2} \mathrm{AQN}(2 \mathrm{~mol} \%)$-catalyzed $\boldsymbol{R}-(-)-8 j \quad$ reaction which was run with 3.0 eq. of trimethylsilyl cyanide in chloroform at $-50{ }^{\circ} \mathrm{C}$ for $46 \mathrm{~h}$. $[\alpha]_{\mathrm{D}}{ }^{25}=-16.8$ (c 2.16, $\left.\mathrm{CHCl}_{3}\right) ;{ }^{1} \mathrm{H}$ NMR $(400 \mathrm{MHz}$, $\left.\mathrm{CDCl}_{3}\right) \delta 0.25(\mathrm{~s}, 9 \mathrm{H}), 0.92-0.98(\mathrm{~m}, 6 \mathrm{H}), 1.56(\mathrm{~s}, 3 \mathrm{H}), 1.60-1.69(\mathrm{~m}, 4 \mathrm{H}), 3.50-3.58(\mathrm{~m}$, $2 \mathrm{H}), 3.68-3.75(\mathrm{~m}, 2 \mathrm{H}), 4.33(\mathrm{~s}, 1 \mathrm{H}) ;{ }^{13} \mathrm{C} \mathrm{NMR}\left(100 \mathrm{MHz}, \mathrm{CDCl}_{3}\right) \delta 1.48,10.74,10.79$, 23.10, 23.14, 23.26, 72.04, 72.37, 72.72, 105.37, 120.98; IR (neat) v 2964, $1463 \mathrm{~cm}^{-1}$; HRMS (DEI) Calcd for $\mathrm{C}_{13} \mathrm{H}_{28} \mathrm{NO}_{3} \mathrm{Si}\left(\mathrm{MH}^{+}\right)$274.1838, found 274.1833.

$\boldsymbol{S}$-(+)-8j was obtained as a colorless oil in $95 \%$ isolated yield and $96 \%$ ee from a (DHQD) $)_{2}$ PHAL (10 mol\%)-catalyzed reaction which was run with 2.0 eq. of trimethylsilyl cyanide in ethyl acetate at $-50{ }^{\circ} \mathrm{C}$ for $88 \mathrm{~h}$.

NC OTMS (+)-8k was obtained as a colorless oil in $96 \%$ isolated yield and

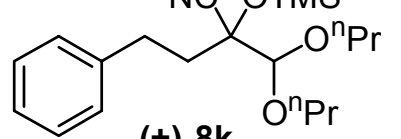
$97 \%$ ee [determined by converting this compound to its corresponding $\beta$-aminoalcohol derivative $\mathbf{8 k \mathbf { D }}$ (vide infra)] from

$(+)-8 k$ a (DHQ) ${ }_{2}$ AQN (20 mol\%)-catalyzed reaction which was run with 3.0 eq. of trimethylsilyl cyanide in chloroform at $-50{ }^{\circ} \mathrm{C}$ for $24 \mathrm{~h} .[\alpha]_{\mathrm{D}}{ }^{25}=2.7$ (c 1.65 , $\left.\mathrm{CHCl}_{3}\right) ;{ }^{1} \mathrm{H} \mathrm{NMR}\left(400 \mathrm{MHz}, \mathrm{CDCl}_{3}\right) \delta 0.29(\mathrm{~s}, 9 \mathrm{H}), 0.95(\mathrm{t}, J=7.2 \mathrm{~Hz}, 3 \mathrm{H}), 0.96(\mathrm{t}, J=$ $7.2 \mathrm{~Hz}, 3 \mathrm{H}), 1.60-1.70(\mathrm{~m}, 4 \mathrm{H}), 2.10-2.15(\mathrm{~m}, 2 \mathrm{H}), 2.80-2.86(\mathrm{~m}, 2 \mathrm{H}), 3.48-3.60(\mathrm{~m}, 2 \mathrm{H})$, 3.69-3.75 (m, 2H), $4.38(\mathrm{~s}, 1 \mathrm{H}), 7.20-7.31(\mathrm{~m}, 5 \mathrm{H}) ;{ }^{13} \mathrm{C} \mathrm{NMR}\left(100 \mathrm{MHz}, \mathrm{CDCl}_{3}\right) \delta 1.62$, $10.81,10.88,23.22,23.28,30.32,38.32,72.16,72.25,75.94,105.01,120.03,126.29$, 128.67, 128.70, 141.35; IR (neat) $v$ 2964, 1604, 1497, $1456 \mathrm{~cm}^{-1} ; \mathrm{HRMS}\left(\mathrm{DCI} / \mathrm{NH}_{3}\right)$ Calcd for $\mathrm{C}_{20} \mathrm{H}_{37} \mathrm{~N}_{2} \mathrm{O}_{3} \mathrm{Si}\left(\mathrm{MNH}_{4}^{+}\right)$381.2573, found 381.2561.

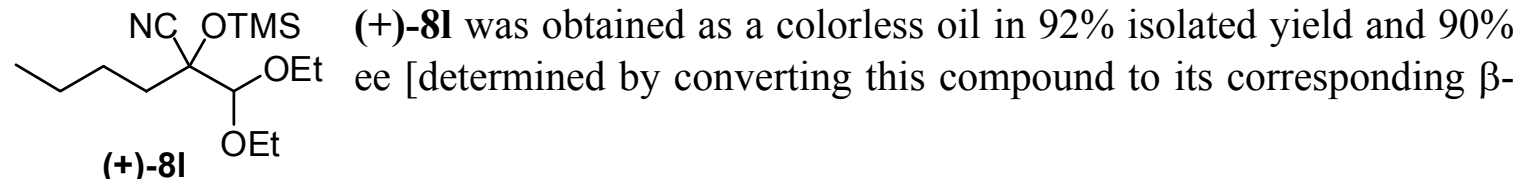


aminoalcohol derivative 8ID (vide infra)] from a (DHQ) $2 \mathrm{AQN}$ (5 mol\%)-catalyzed reaction which was run with 3.0 eq. of trimethylsilyl cyanide in chloroform at $-50{ }^{\circ} \mathrm{C}$ for 18 h. $[\alpha]_{\mathrm{D}}{ }^{25}=0.20\left(\mathrm{c} 1.50, \mathrm{CHCl}_{3}\right) ;{ }^{1} \mathrm{H} \mathrm{NMR}\left(400 \mathrm{MHz}, \mathrm{CDCl}_{3}\right) \delta 0.25(\mathrm{~s}, 9 \mathrm{H}), 0.94(\mathrm{t}$, $J=7.0 \mathrm{~Hz}, 3 \mathrm{H}), 1.26(\mathrm{t}, J=7.0 \mathrm{~Hz}, 3 \mathrm{H}), 1.27(\mathrm{t}, J=7.0 \mathrm{~Hz}, 3 \mathrm{H}), 1.33-1.41(\mathrm{~m}, 2 \mathrm{H})$, $1.45-1.52(\mathrm{~m}, 2 \mathrm{H}), 1.76-1.83(\mathrm{~m}, 2 \mathrm{H}), 3.60-3.69(\mathrm{~m}, 2 \mathrm{H}), 3.78-3.87(\mathrm{~m}, 2 \mathrm{H}), 4.33(\mathrm{~s}$, $1 \mathrm{H}) ;{ }^{13} \mathrm{C} \mathrm{NMR}\left(100 \mathrm{MHz}, \mathrm{CDCl}_{3}\right) \delta 1.52,14.13,15.32,15.39,22.78,25.90,36.01$, 65.79, 66.00, 76.16, 104.87, 120.14; IR (neat) $\vee 2962,1453 \mathrm{~cm}^{-1} ; \mathrm{HRMS}\left(\mathrm{DCI} / \mathrm{NH}_{3}\right)$ Calcd for $\mathrm{C}_{14} \mathrm{H}_{33} \mathrm{~N}_{2} \mathrm{O}_{3} \mathrm{Si}\left(\mathrm{MNH}_{4}{ }^{+}\right)$305.2260, found 305.2263.

NC OTMS (+)-8m was obtained as a colorless oil in $81 \%$ isolated yield and $94 \%$ ee OEt [determined by converting this compound to its corresponding $\beta$ aminoalcohol derivative $\mathbf{8 m D}$ (vide infra)] from a (DHQ) ${ }_{2} \mathrm{AQN}$ (20 mol\%)-catalyzed reaction which was run with 3.0 eq. of trimethylsilyl

$(+)-8 m$ cyanide in chloroform at $-40{ }^{\circ} \mathrm{C}$ for $94 \mathrm{~h} .[\alpha]_{\mathrm{D}}{ }^{25}=0.41\left(\mathrm{c} 1.45, \mathrm{CHCl}_{3}\right)$; ${ }^{1} \mathrm{H}$ NMR (400 MHz, $\left.\mathrm{CDCl}_{3}\right) \delta 0.25(\mathrm{~s}, 9 \mathrm{H}), 0.99(\mathrm{~d}, J=6.8 \mathrm{~Hz}, 3 \mathrm{H}), 1.07$ (d, $J=6.8 \mathrm{~Hz}$, $3 \mathrm{H}), 1.27(\mathrm{t}, J=7.0 \mathrm{~Hz}, 6 \mathrm{H}), 2.15(\mathrm{sp}, J=6.8 \mathrm{~Hz}, 1 \mathrm{H}), 3.60-3.70(\mathrm{~m}, 2 \mathrm{H}), 3.76-3.89(\mathrm{~m}$, 2H), $4.42(\mathrm{~s}, 1 \mathrm{H}) ;{ }^{13} \mathrm{C} \mathrm{NMR}\left(100 \mathrm{MHz}, \mathrm{CDCl}_{3}\right) \delta 1.65,15.37,15.38,16.60,18.09,34.54$, 65.35, 65.42, 79.64, 103.29, 119.49; IR (neat) $\vee 2977,1447 \mathrm{~cm}^{-1}$; HRMS (DCI/NH $\left.\mathrm{NH}_{3}\right)$ Calcd for $\mathrm{C}_{13} \mathrm{H}_{31} \mathrm{~N}_{2} \mathrm{O}_{3} \mathrm{Si}\left(\mathrm{MNH}_{4}^{+}\right)$291.2104, found 291.2114.

General procedure for the conversion of silylcyanohydrin to its corresponding $\beta$ aminoalcohol derivative and determination of ee

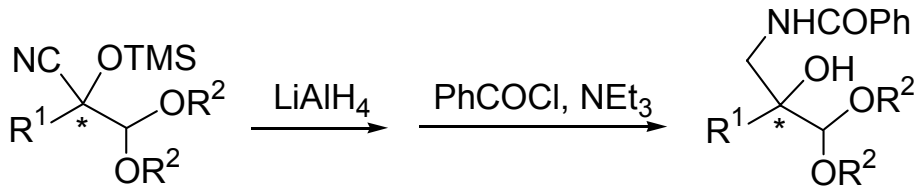

To a solution of silylcyanohydrin $(0.10 \mathrm{mmol})$ in dry ether $(15.0 \mathrm{~mL})$ at room temperature was added $\mathrm{LiAlH}_{4}(0.30 \mathrm{mmol})$. The mixture was stirred at that temperature for $1.0 \mathrm{~h}$, and cooled with ice-water bath. Water $(0.20 \mathrm{~mL})$ was slowly added and the resulting the mixture was filtered. The filtrate was dried over anhydrous potassium carbonate, and then filtered. To this filtrate was added triethyl amine $(0.60 \mathrm{mmol})$ and benzoyl chloride $(0.12 \mathrm{mmol})$. After being stirred at room temperature for $0.5 \mathrm{~h}$, the mixture was filtered and concentrated. The residue was purified by silica gel column chromatography (acetone: hexanes/1:20 to 1:10) to give the amide.

\section{1) $\beta$-aminoalcohol derivatives from the products of Table 1 (Entries 2-4)}

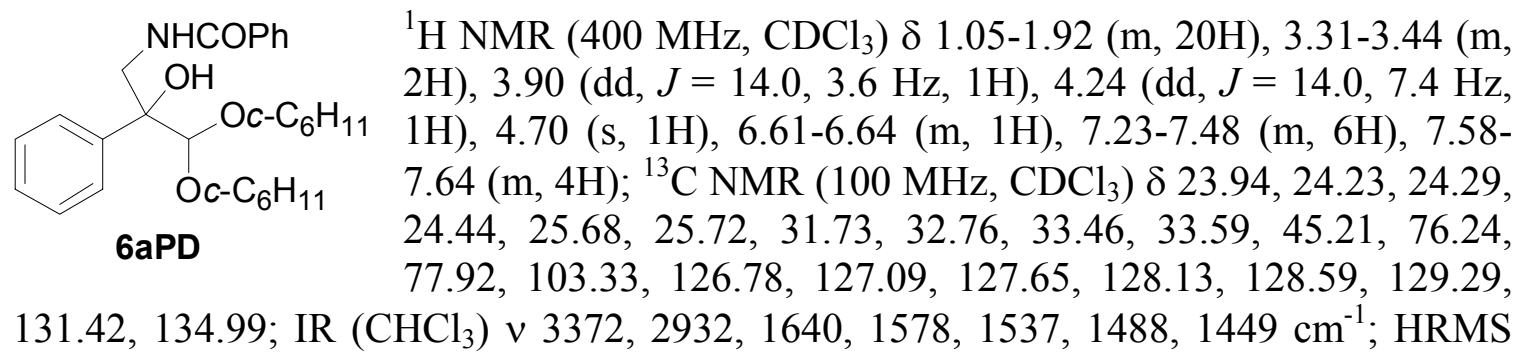


(EI) Calcd for $\mathrm{C}_{28} \mathrm{H}_{37} \mathrm{O}_{4} \mathrm{~N}\left(\mathrm{MH}^{+}\right)$451.2723, found 451.2733. HPLC conditions: Daicel Chiralpak OD, isopropanol : hexane (1:99), $1.0 \mathrm{~mL} / \mathrm{min}, \lambda=254 \mathrm{~nm}$; for the derivative from the product of $(\mathrm{DHQ})_{2} \mathrm{AQN}$-catalyzed reaction, $\mathrm{t}$ (major) $=30.3 \mathrm{~min}, \mathrm{t}$ (minor) $=$ $39.6 \mathrm{~min}$.

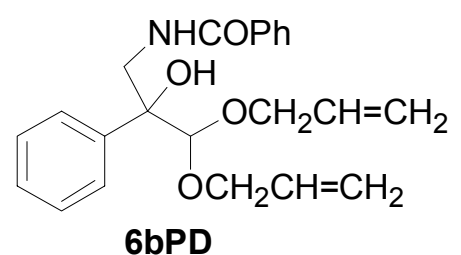

${ }^{1} \mathrm{H}$ NMR (400 MHz, $\left.\mathrm{CDCl}_{3}\right) \delta$ 3.86-3.91 (m, 1H), 3.97-4.21 $(\mathrm{m}, 5 \mathrm{H}), 4.60(\mathrm{~s}, 1 \mathrm{H}), 5.12-5.25(\mathrm{~m}, 4 \mathrm{H}), 5.76-5.88(\mathrm{~m}, 2 \mathrm{H})$, 6.58-6.62 (m, 1H), 7.25-7.62 (m, 10H); ${ }^{13} \mathrm{C} \mathrm{NMR} \mathrm{(100} \mathrm{MHz,}$ $\left.\mathrm{CDCl}_{3}\right) \delta 45.38,71.01,71.48,78.19,106.38,117.78,117.94$, $126.61,127.11,127.80,128.33,128.62,131.57,133.91$, 134.02, 134.64, 140.56, 168.60; IR (neat) v 3421, 3061, 1713, 1644, 1603, 1578, 1538, 1489, $1450 \mathrm{~cm}^{-1}$; HRMS (EI) Calcd for $\mathrm{C}_{22} \mathrm{H}_{26} \mathrm{O}_{4} \mathrm{~N}\left(\mathrm{MH}^{+}\right)$368.1862, found 368.1832. HPLC conditions: Daicel Chiralpak OD, isopropanol : hexane $(5: 95), 1.0 \mathrm{~mL} / \mathrm{min}, \lambda=$ $254 \mathrm{~nm}$; for the derivative from the product of (DHQ) ${ }_{2} \mathrm{AQN}$-catalyzed reaction, $\mathrm{t}$ (major) $=33.3 \mathrm{~min}, \mathrm{t}$ (minor) $=23.9 \mathrm{~min}$.

$\begin{array}{cl}\mathrm{NHCOPh}_{\mathrm{OH}} & { }^{1} \mathrm{H} \mathrm{NMR}\left(400 \mathrm{MHz}, \mathrm{CDCl}_{3}\right) \delta 0.88(\mathrm{t}, J=7.0 \mathrm{~Hz}, 6 \mathrm{H}), 1.19-1.31 \\ (\mathrm{~m}, 20 \mathrm{H}), 1.49-1.57(\mathrm{~m}, 4 \mathrm{H}), 3.23-3.29(\mathrm{~m}, 1 \mathrm{H}), 3.39-3.44(\mathrm{~m}, \\ \mathrm{On}_{-} \mathrm{C}_{8} \mathrm{H}_{17} & 1 \mathrm{H}), 3.66-3.73(\mathrm{~m}, 2 \mathrm{H}), 3.94(\mathrm{dd}, J=14.4,3.8 \mathrm{~Hz}, 1 \mathrm{H}), 4.21(\mathrm{dd}, \\ \mathrm{O}=14.4,7.0 \mathrm{~Hz}, 1 \mathrm{H}), 4.48(\mathrm{~s}, 1 \mathrm{H}), 6.68-6.72(\mathrm{~m}, 1 \mathrm{H}), 7.25-7.65 \\ \mathrm{O}-\mathrm{C}_{8} \mathrm{H}_{17} & (\mathrm{~m}, 10 \mathrm{H}) ;{ }^{13} \mathrm{C} \mathrm{NMR}\left(100 \mathrm{MHz}, \mathrm{CDCl}_{3}\right) \delta 14.30,22.85,26.22, \\ \text { 6cPD } & 26.25,29.40,29.54,29.93,30.10,32.01,45.39,70.83,71.38,\end{array}$
$77.90,107.90,126.52,127.12,127.71,128.28,128.62,131.56,135.00,140.67,168.27$; IR $\left(\mathrm{CHCl}_{3}\right) \vee 2924,1688,1603,1584,1454 \mathrm{~cm}^{-1}$; HRMS (EI) Calcd for $\mathrm{C}_{32} \mathrm{H}_{49} \mathrm{NO}_{4}\left(\mathrm{M}^{+}\right)$ 511.3662, found 511.3720. HPLC conditions: Daicel Chiralpak OD, isopropanol : hexane ( $5: 95), 1.0 \mathrm{~mL} / \mathrm{min}, \lambda=254 \mathrm{~nm}$; for the derivative from the product of (DHQ) ${ }_{2} \mathrm{AQN}-$ catalyzed reaction, $\mathrm{t}_{\mathrm{r}}($ major $)=7.1 \mathrm{~min}, \mathrm{t}_{\mathrm{r}}($ minor $)=8.9 \mathrm{~min}$.

\section{2) $\beta$-aminoalcohol derivatives from the products of Table 2}<smiles>CCOC(OCC)C(CN)(CNC(=O)c1ccccc1)c1ccccc1</smiles>

$8 \mathrm{aD}$

8aD was obtained as a colorless oil in $81 \%$ isolated yield. ${ }^{1} \mathrm{H}$ NMR $\left(400 \mathrm{MHz}, \mathrm{CDCl}_{3}\right) \delta 1.13-1.21(\mathrm{~m}, 6 \mathrm{H}), 3.28-3.37(\mathrm{~m}, 1 \mathrm{H}), 3.43-3.51$ $(\mathrm{m}, 1 \mathrm{H}), 3.68-3.81(\mathrm{~m}, 2 \mathrm{H}), 3.93(\mathrm{dd}, J=14.2,4.0 \mathrm{~Hz}, 1 \mathrm{H}), 4.18(\mathrm{dd}, J$ $=14.2,6.6 \mathrm{~Hz}, 1 \mathrm{H}), 4.47(\mathrm{~s}, 1 \mathrm{H}), 6.62(\mathrm{~s}$, br., $1 \mathrm{H}), 7.24-7.45(\mathrm{~m}, 6 \mathrm{H})$, 7.58-7.62 (m, 4H); ${ }^{13} \mathrm{C}$ NMR $\left(100 \mathrm{MHz}, \mathrm{CDCl}_{3}\right) \delta 15.34,15.52,45.17$, $66.25,65.54,77.75,107.64,126.53,127.03,127.67,128.23,128.58$, 131.44, 134.80, 140.67, 168.27; IR (neat) v 3356, 2976, 1714, 1643, $1535 \mathrm{~cm}^{-1}$; HRMS (EI) Calcd for $\mathrm{C}_{20} \mathrm{H}_{26} \mathrm{NO}_{4}\left(\mathrm{MH}^{+}\right)$344.1862, found 344.1839. HPLC conditions: Daicel Chiralpak OD, isopropanol:hexane $/ 15: 85,1.0 \mathrm{~mL} / \mathrm{min}, \lambda=254 \mathrm{~nm}$; for the derivative from the product of $(\mathrm{DHQ})_{2} \mathrm{AQN}$-catalyzed reaction, $\mathrm{t}_{\mathrm{r}}$ (major $)=6.1 \mathrm{~min}, \mathrm{t}_{\mathrm{r}}($ minor $)=7.1$ $\min ]$.

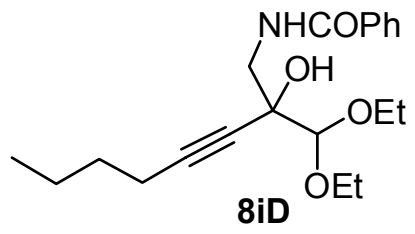

8iD was obtained as a colorless oil in 59\% isolated yield. ${ }^{1} \mathrm{H}$ $\operatorname{NMR}\left(400 \mathrm{MHz}, \mathrm{CDCl}_{3}\right) \delta 0.84(\mathrm{t}, J=7.2 \mathrm{~Hz}, 3 \mathrm{H}), 1.27(\mathrm{t}, J=$ $7.0 \mathrm{~Hz}, 6 \mathrm{H}), 1.32-1.49(\mathrm{~m}, 4 \mathrm{H}), 2.21(\mathrm{t}, J=6.8 \mathrm{~Hz}, 2 \mathrm{H}), 3.68-$ 
$3.76(\mathrm{~m}, 2 \mathrm{H}), 3.80(\mathrm{~d}, J=5.2 \mathrm{~Hz}, 2 \mathrm{H}), 3.84-3.93(\mathrm{~m}, 2 \mathrm{H}), 4.47(\mathrm{~s}, 1 \mathrm{H}), 6.76-6.81(\mathrm{~m}$, $1 \mathrm{H}), 7.39-7.53(\mathrm{~m}, 3 \mathrm{H}), 7.80(\mathrm{~d}, J=8.0 \mathrm{~Hz}, 2 \mathrm{H}) ;{ }^{13} \mathrm{C} \mathrm{NMR}\left(100 \mathrm{MHz}, \mathrm{CDCl}_{3}\right) \delta 13.68$, $15.51,18.60,22.04,30.66,45.78,66.21,66.35,72.66,78.71,87.17,105.85,127.17$, 128.66, 131.61, 134.78, 168.21; IR (neat) $v 3356,2932,2243,1712,1643,1535 \mathrm{~cm}^{-1}$; HRMS (EI) Calcd for $\mathrm{C}_{20} \mathrm{H}_{30} \mathrm{NO}_{4}\left(\mathrm{MH}^{+}\right)$348.2175, found 348.2195. HPLC conditions: Daicel Chiralpak AD, isopropanol:hexane $/ 5: 95,1.0 \mathrm{~mL} / \mathrm{min}, \lambda=254 \mathrm{~nm}$; for the derivative from the product of $(\mathrm{DHQ})_{2} \mathrm{AQN}$-catalyzed reaction, $\mathrm{t}_{\mathrm{r}}($ major $)=12.7 \mathrm{~min}, \mathrm{t}_{\mathrm{r}}$ $($ minor $)=28.9 \mathrm{~min}]$.

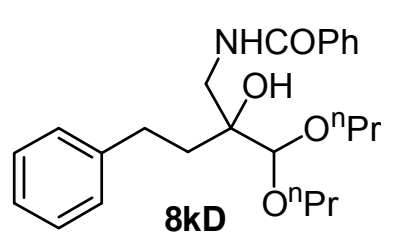

8kD was obtained as a colorless oil in $41 \%$ isolated yield. ${ }^{1} \mathrm{H}$ NMR $\left(400 \mathrm{MHz}, \mathrm{CDCl}_{3}\right) \delta 0.95(\mathrm{t}, J=7.0 \mathrm{~Hz}, 3 \mathrm{H}), 0.96(\mathrm{t}, J=$ $7.0 \mathrm{~Hz}, 3 \mathrm{H}), 1.59-1.71(\mathrm{~m}, 4 \mathrm{H}), 1.88-1.93(\mathrm{~m}, 2 \mathrm{H}), 2.76-2.82(\mathrm{~m}$, $2 \mathrm{H}), 3.45$ (dt, $J=8.4,6.8 \mathrm{~Hz}, 1 \mathrm{H}), 3.59(\mathrm{dt}, J=9.2,6.8 \mathrm{~Hz}, 1 \mathrm{H})$, $3.72(\mathrm{~d}, J=5.6 \mathrm{~Hz}, 2 \mathrm{H}), 3.72-3.84(\mathrm{~m}, 2 \mathrm{H}), 4.40(\mathrm{~s}, 1 \mathrm{H}), 7.00(\mathrm{~s}$, br., 1H), 7.17-7.29 (m, 5H), 7.41-7.52 (m, 3H), 7.77-7.81 (m, 2H); ${ }^{13} \mathrm{C}$ NMR (100 MHz, $\left.\mathrm{CDCl}_{3}\right) \delta 10.82,10.88,23.49,23.60,29.50,36.83,44.05,72.81,73.62,75.29,108.20$, $125.96,127.10,128.59,128.63,128.71,131.61,134.83,142.70,168.01$; IR (neat) $v$ $3393,2962,1712,1642,1534,1489,1452 \mathrm{~cm}^{-1}$; HRMS (EI) Calcd for $\mathrm{C}_{24} \mathrm{H}_{34} \mathrm{NO}_{4}\left(\mathrm{MH}^{+}\right)$ 400.2488, found 400.2495. HPLC conditions: Daicel Chiralpak AD, isopropanol:hexane $/ 15: 85,1.0 \mathrm{~mL} / \mathrm{min}, \lambda=254 \mathrm{~nm}$; for the derivative from the product of $(\mathrm{DHQ})_{2} \mathrm{AQN}$-catalyzed reaction, $\mathrm{t}_{\mathrm{r}}($ major $)=5.9 \mathrm{~min}, \mathrm{t}_{\mathrm{r}}($ minor $\left.)=8.7 \mathrm{~min}\right]$.

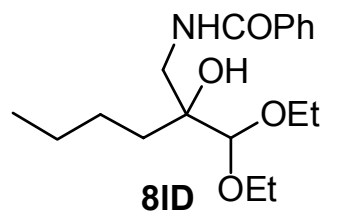

8ID was obtained as a colorless oil in $89 \%$ isolated yield. ${ }^{1} \mathrm{H}$ NMR $\left(400 \mathrm{MHz}, \mathrm{CDCl}_{3}\right) \delta 0.90(\mathrm{t}, J=7.2 \mathrm{~Hz}, 3 \mathrm{H}), 1.27(\mathrm{t}, J=7.0 \mathrm{~Hz}$, $3 \mathrm{H}), 1.28(\mathrm{t}, J=7.0 \mathrm{~Hz}, 3 \mathrm{H}), 1.25-1.35(\mathrm{~m}, 2 \mathrm{H}), 1.36-1.46(\mathrm{~m}, 2 \mathrm{H})$, 1.53-1.63 (m, 2H), $2.54(\mathrm{~s}$, br., $1 \mathrm{H}), 3.57-3.70(\mathrm{~m}, 4 \mathrm{H}), 3.85-3.93(\mathrm{~m}$, $2 \mathrm{H}), 4.37(\mathrm{~s}, 1 \mathrm{H}), 6.96-7.03(\mathrm{~m}, 1 \mathrm{H}), 7.39-7.51(\mathrm{~m}, 3 \mathrm{H}), 7.76-7.81(\mathrm{~m}, 2 \mathrm{H}) ;{ }^{13} \mathrm{C} \mathrm{NMR}$ $\left(100 \mathrm{MHz}, \mathrm{CDCl}_{3}\right) \delta 14.17,15.67,15.73,23.46,24.99,34.20,43.99,66.51,67.00,74.96$, 107.98, 127.02, 128.60, 131.44, 134.89, 167.71; IR (neat) $\vee 3400,2957,1644,1535 \mathrm{~cm}^{-1}$; HRMS (EI) Calcd for $\mathrm{C}_{18} \mathrm{H}_{30} \mathrm{NO}_{4}\left(\mathrm{MH}^{+}\right) 324.2175$, found 324.2174. HPLC conditions: Daicel Chiralpak AD, isopropanol:hexane $/ 5: 95,1.0 \mathrm{~mL} / \mathrm{min}, \lambda=254 \mathrm{~nm}$; for the derivative from the product of $(\mathrm{DHQ})_{2} \mathrm{AQN}$-catalyzed reaction, $\mathrm{t}_{\mathrm{r}}($ major $)=10.4 \mathrm{~min}, \mathrm{t}_{\mathrm{r}}$ $($ minor $)=13.1 \mathrm{~min}]$.

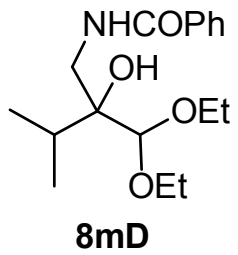

8mD was obtained as a colorless oil in $86 \%$ isolated yield. ${ }^{1} \mathrm{H}$ NMR (400 $\left.\mathrm{MHz}, \mathrm{CDCl}_{3}\right) \delta 1.00(\mathrm{~d}, J=6.8 \mathrm{~Hz}, 3 \mathrm{H}), 1.01(\mathrm{~d}, J=6.8 \mathrm{~Hz}, 3 \mathrm{H}), 1.27(\mathrm{t}$, $J=7.0 \mathrm{~Hz}, 1 \mathrm{H}), 1.28(\mathrm{p}, J=7.0 \mathrm{~Hz}, 3 \mathrm{H}), 2.01(\mathrm{sp}, J=6.8 \mathrm{~Hz}, 1 \mathrm{H}), 3.54-$ $3.75(\mathrm{~m}, 4 \mathrm{H}), 3.85-3.96(\mathrm{~m}, 2 \mathrm{H}), 4.51(\mathrm{~s}, 1 \mathrm{H}), 7.14-7.19(\mathrm{~m}, 1 \mathrm{H}), 7.38-$ $7.51(\mathrm{~m}, 3 \mathrm{H}), 7.76-7.81(\mathrm{~m}, 2 \mathrm{H}) ;{ }^{13} \mathrm{C}$ NMR $\left(100 \mathrm{MHz}, \mathrm{CDCl}_{3}\right) \delta 15.65$, $15.81,17.04,17.13,42.47,65.99,67.58,76.00,107.32,127.02,128.57$, 131.40, 134.90, 167.62; IR (neat) $v$ 3399, 2975, 1717, 1645, $1535 \mathrm{~cm}^{-1}$; HRMS (EI) Calcd for $\mathrm{C}_{17} \mathrm{H}_{28} \mathrm{NO}_{4}\left(\mathrm{MH}^{+}\right)$310.2018, found 310.1998. HPLC conditions: Daicel Chiralpak OD + Hypersil SI, isopropanol:hexane $/ 2: 98,1.0 \mathrm{~mL} / \mathrm{min}, \lambda=254 \mathrm{~nm}$; for the derivative from the product of $(\mathrm{DHQ})_{2} \mathrm{AQN}$-catalyzed reaction, $\mathrm{t}_{\mathrm{r}}($ major $)=22.9 \mathrm{~min}, \mathrm{t}_{\mathrm{r}}$ $($ minor $)=18.2 \mathrm{~min}]$. 
Preparation of optically active tertiary silylcyanohydrin on a $100 \mathrm{mmol}$ scale and the recovery of catalyst

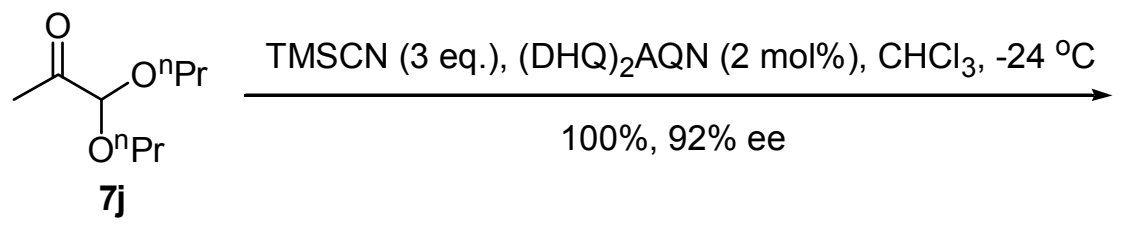<smiles>CCCOC(O[Na])C(C)(C#N)O[Na]</smiles>

$\boldsymbol{R}-(-)-8 \mathrm{j}$

$(100 \mathrm{mmol}, 27.3 \mathrm{~g})$

To a solution of trimethylsilyl cyanide $(29.7 \mathrm{~g}, 300 \mathrm{mmol})$ and (DHQ) ${ }_{2} \mathrm{AQN}(1.71$ g, $2.00 \mathrm{mmol})$ in chloroform $(100 \mathrm{~mL})$ at $-50{ }^{\circ} \mathrm{C}$ was added dropwise $\alpha, \alpha-$ dipropoxyacetone $(17.4 \mathrm{~g}, 100 \mathrm{mmol})$. The mixture was stirred at that temperature for 72 h. Methanol $(20 \mathrm{~mL})$ was slowly added to the mixture, and after 5 min the mixture was diluted with hexanes $(400 \mathrm{~mL})$. The mixture was washed successively with aqueous $\mathrm{HCl}$ $(0.2 \mathrm{~N}, 2 \times 50 \mathrm{~mL})$ and water $(3 \times 30 \mathrm{~mL})$. The organic layer was dried over anhydrous sodium sulfate and concentrated to give the silylcyanohydrin $(27.3 \mathrm{~g}, 100 \%, 92 \% \mathrm{ee})$ as a colorless oil.

To the aqueous layer was added $\mathrm{K}_{2} \mathrm{CO}_{3}$ to adjust the $\mathrm{pH}$ value of the solution to 911. The resulting mixture was extracted with ethyl acetate $(150 \mathrm{~mL})$, and the organic layer was washed with brine, dried over anhydrous sodium sulfate, concentrated to afford the recovered catalyst (DHQ) ${ }_{2} \mathrm{AQN}$ in quantitative yield, which is shown to be pure by ${ }^{1} \mathrm{H}$ NMR (400 MHz, $\mathrm{CDCl}_{3}$ ) (see more details in the General Procedure).

Conversion of (-)-9j to a chiral $\beta$-amino acid and the determination of its absolute configuration of (-)-8j

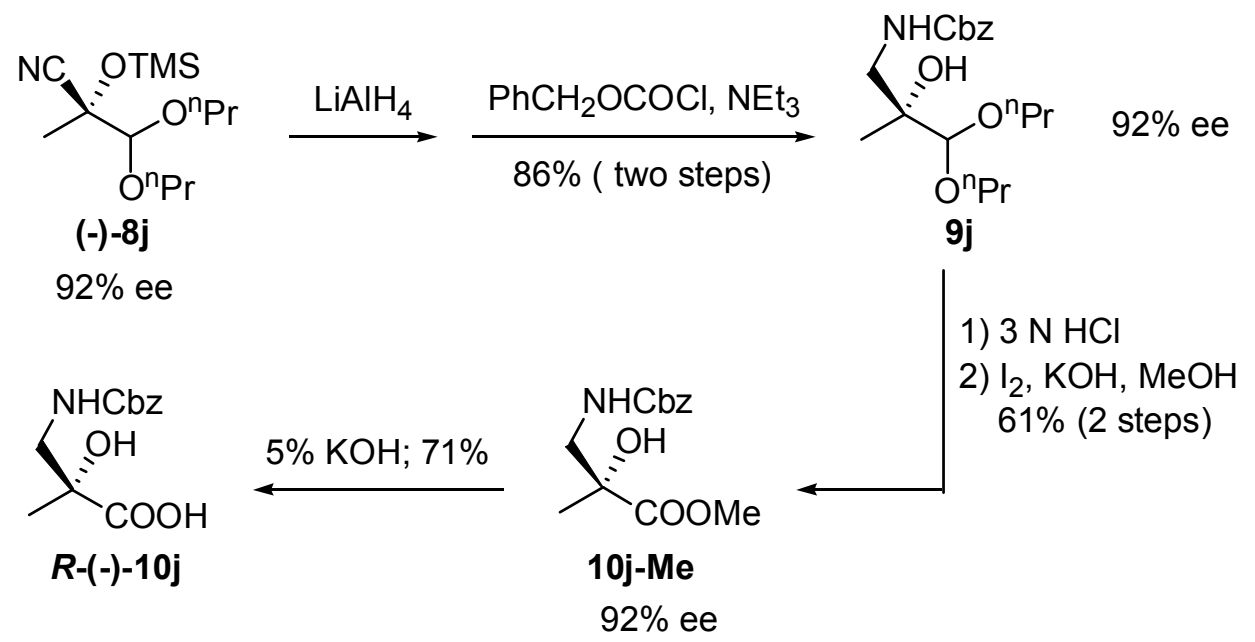

(-)-8j was obtained in 92\% ee from a (DHQ) ${ }_{2} \mathrm{AQN}$-catalyzed reaction. (-)-8j $(1.37 \mathrm{~g}, 5.00 \mathrm{mmol})$ was reduced to its corresponding $\beta$-aminoalcohol with $\mathrm{LiAlH}_{4}$ using the same procedure as described above. To a solution of the crude $\beta$-aminoalcohol in ether $(100 \mathrm{~mL})$ at room temperature was added triethylamine $(1.11 \mathrm{~g}, 11.0 \mathrm{mmol})$ and benzyl chloroformate $(940 \mathrm{mg}, 5.10 \mathrm{mmol})$. The mixture was stirred for $0.5 \mathrm{~h}$. The 
mixture was washed with brine, dried over magnesium sulfate and then concentrated. The residue was purified by silica gel column chromatography (acetone:hexanes/1:20 to 1:10) to give carbamate $9 \mathbf{j}$ ( $1.47 \mathrm{~g}, 86 \%$ for two steps) as a colorless oil. ${ }^{7}$ The ee of $9 \mathbf{j}$ was determined to be $92 \%$ [HPLC conditions: Daicel Chiralpak AD + Hypersil SI, isopropanol:hexane $/ 1: 99,0.50 \mathrm{~mL} / \mathrm{min}, \mathrm{t}_{\mathrm{r}}$ (major) $=113.3 \mathrm{~min}, \mathrm{t}_{\mathrm{r}}($ minor $\left.)=101.7 \mathrm{~min}\right]$. $[\alpha]_{\mathrm{D}}{ }^{25}=1.6\left(\mathrm{c} 1.12, \mathrm{CHCl}_{3}\right) ;{ }^{1} \mathrm{H} \mathrm{NMR}\left(400 \mathrm{MHz}, \mathrm{CDCl}_{3}\right) \delta 0.94(\mathrm{t}, J=7.4 \mathrm{~Hz}, 6 \mathrm{H}), 1.17$ $(\mathrm{s}, 3 \mathrm{H}), 1.58-1.68(\mathrm{~m}, 4 \mathrm{H}), 2.45(\mathrm{~s}, 1 \mathrm{H}), 3.30-3.36(\mathrm{~m}, 2 \mathrm{H}), 3.43-3.50(\mathrm{~m}, 2 \mathrm{H}), 3.71-3.79$ $(\mathrm{m}, 2 \mathrm{H}), 4.24(\mathrm{~s}, 1 \mathrm{H}), 5.11(\mathrm{~s}, 2 \mathrm{H}), 5.25-5.30(\mathrm{~m}, 1 \mathrm{H}), 7.39-7.39(\mathrm{~m}, 5 \mathrm{H}) ;{ }^{13} \mathrm{C} \mathrm{NMR}(100$ $\left.\mathrm{MHz}_{,} \mathrm{CDCl}_{3}\right) \delta 10.79,20.65,23.43,46.73,66.84,72.78,72.82,74.08,107.77,127.20$, $128.22,128.68,136.91,157.13$.

To a solution of $9 \mathbf{j}(495 \mathrm{mg}, 1.46 \mathrm{mmol})$ in acetone $(20 \mathrm{~mL})$ at room temperature was added $3 \mathrm{~N}$ aqueous $\mathrm{HCl}(4.0 \mathrm{~mL})$. The mixture was stirred for $3 \mathrm{~h}$. After being quenched with aqueous potassium carbonate ( $828 \mathrm{mg}$ in $4 \mathrm{~mL}$ of water), acetone was removed under reduced pressure. The mixture was extracted with ethyl acetate $(2 \times 20$ $\mathrm{mL}$ ), and the organic layer was dried over anhydrous magnesium sulfate, concentrated. The residue was dissolved in methanol $(50 \mathrm{~mL})$ and cooled to $0{ }^{\circ} \mathrm{C}$. To this mixture was added $\mathrm{KOH}(212 \mathrm{mg}, 3.79 \mathrm{mmol})$ and then $\mathrm{I}_{2}(480 \mathrm{mg}, 1.90 \mathrm{mmol}){ }^{8}$ The mixture was stirred for $30 \mathrm{~min}$, and added dropwise $1 \mathrm{~N}$ aqueous $\mathrm{HCl}(4.5 \mathrm{~mL})$. Saturated aqueous sodium thiosulfate was added until the mixture turned colorless. Methanol was removed under reduced pressure and the mixture was extracted with ethyl acetate $(3 \times 20 \mathrm{~mL})$. The organic layer was dried over anhydrous sodium sulfate, concentrated. The residue was purified by silica gel column chromatography (acetone:hexanes/1:10 to $1: 5$ ) to give $\mathbf{1 0 j}$ Me (238 mg, 61\% for two steps) as a colorless oil. The ee of $\mathbf{1 0 j}$-Me was determined to be 92\% [HPLC conditions: Daicel Chiralpak OD, isopropanol:hexane $/ 10: 90,1.0 \mathrm{~mL} / \mathrm{min}$, $\mathrm{t}_{\mathrm{r}}($ major $)=13.3 \mathrm{~min}, \mathrm{t}_{\mathrm{r}}($ minor $\left.)=15.3 \mathrm{~min}\right] .[\alpha]_{\mathrm{D}}{ }^{25}=-18.9\left(\mathrm{c} 1.95, \mathrm{CHCl}_{3}\right) ;{ }^{1} \mathrm{H} \mathrm{NMR}$ (400 MHz, $\mathrm{CDCl}_{3}, 3.5: 1$ mixture of rotamers) $\delta 1.39(\mathrm{~s}, 3 \mathrm{H}), 3.26(\mathrm{dd}, J=14.0,5.2 \mathrm{~Hz}$, $1 \mathrm{H}), 3.65(\mathrm{dd}, J=14.0,7.6 \mathrm{~Hz}, 1 \mathrm{H}), 3.72(\mathrm{~s}, 3 \mathrm{H}), 5.07$ (s, 2H), 5.36-5.41 (m, 1H), 7.27$7.38(\mathrm{~m}, 5 \mathrm{H}) ;{ }^{1} \mathrm{H}$ NMR $\left(400 \mathrm{MHz}, \mathrm{CDCl}_{3}\right.$, minor rotamer, partial) $\delta 3.78(\mathrm{~s}, 3 \mathrm{H}) ;{ }^{13} \mathrm{C}$ NMR $\left(100 \mathrm{MHz}, \mathrm{CDCl}_{3}\right) \delta 23.32,48.96,53.16,66.98,74.74,128.18,128.24,128.61$, 136.48, 156.74, 176.01; IR (neat) $v$ 3390, 2953, 1732, 1538, $1454 \mathrm{~cm}^{-1}$; HRMS (DCI/ $\left.\mathrm{NH}_{3}\right)$ Calcd for $\mathrm{C}_{13} \mathrm{H}_{18} \mathrm{NO}_{5}\left(\mathrm{MH}^{+}\right)$268.1185, found 268.1195.

To a solution of $\mathbf{1 0 j}-\mathbf{M e}(123 \mathrm{mg}, 0.46 \mathrm{mmol})$ in methanol $(15 \mathrm{~mL})$ at room temperature was added $5 \%$ aqueous $\mathrm{KOH}(7.5 \mathrm{~mL})$. The mixture was stirred for $30 \mathrm{~min}$ and methanol was removed under reduced pressure. The mixture was extracted with ether $(10 \mathrm{~mL})$ and the aqueous layer was acidified with $3 \mathrm{~N}$ aqueous $\mathrm{HCl}$ to $\mathrm{PH}=2$. The mixture was extracted with ethyl acetate $(3 \times 20 \mathrm{~mL})$. The organic layer was dried over anhydrous sodium sulfate, and then concentrated to give $\mathbf{1 0 j}(82.6 \mathrm{mg}, 71 \%)$ as a white solid. $[\alpha]_{\mathrm{D}}{ }^{25}=-9.7$ (c 1.2, DMSO), literature value for $(\boldsymbol{S})-\mathbf{1 0 j}:[\alpha]_{\mathrm{D}}{ }^{25}=9.1$ (c 1.0 , DMSO) $;{ }^{9}{ }^{1} \mathrm{H}$ NMR (400 MHz, DMSO, 3.5:1 mixture of rotamers) $\delta 1.20(\mathrm{~s}, 3 \mathrm{H}), 3.18$ (dd, $J=13.2,5.6 \mathrm{~Hz}, 1 \mathrm{H}), 3.29$ (dd, $J=13.2,6.8 \mathrm{~Hz}, 1 \mathrm{H}), 5.00$ (s, 2H), 7.05-7.09 (m, 1H), 7.26-7.38 (m, 5H); ${ }^{1} \mathrm{H}$ NMR (400 MHz, DMSO, minor rotamer, partial) $\delta 1.51(\mathrm{~s}$, $3 \mathrm{H}), 3.34(\mathrm{~d}, J=9.0 \mathrm{~Hz}, 1 \mathrm{H}), 3.63(\mathrm{~d}, J=9.0 \mathrm{~Hz}, 1 \mathrm{H}), 7.68$ (s, br., $1 \mathrm{H}) ;{ }^{13} \mathrm{C} \mathrm{NMR}(100$

\footnotetext{
${ }^{7}$ Tian, S.-K.; Deng, L. J. Am. Chem. Soc. 2001, 123, 6195.

${ }^{8}$ Yamamoto, K.; Shimizu, M.; Yamada, S. J. Org. Chem. 1992, 57, 33.

${ }^{9}$ Pires, R.; Burger, K. Synthesis 1996, 1277.
} 
$\mathrm{MHz}$, DMSO, mixture of rotamers) $\delta 23.16,48.81,65.32,73.57,127.66,127.78,128.37$, $137.16,156.43,176.13 ;{ }^{13} \mathrm{C}$ NMR (400 MHz, DMSO, minor rotamer, partial) $\delta 22.99$, 49.31, 79.48, 157.67, 172.82 .

By comparing the specific rotation of $\mathbf{1 0 j}$ with known $(\mathbf{S}) \mathbf{- 1 0 j}$, the absolute configuration of (-)-8j was assigned to be $R$.

\section{Conversion of (+)-9j to (-)-11j}

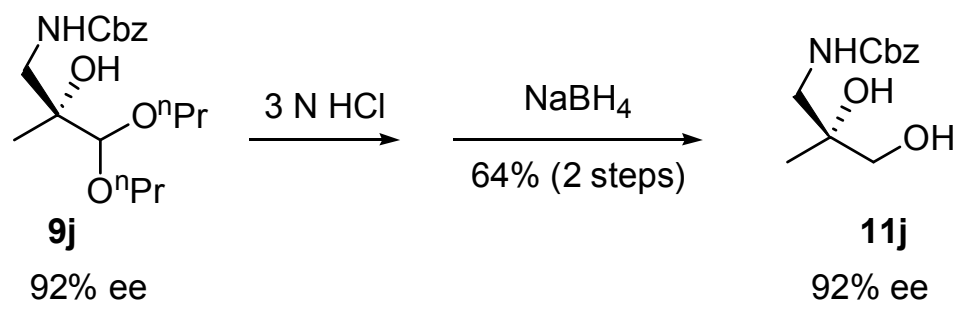

9j (225 mg, $0.66 \mathrm{mmol})$ was hydrolyzed with $3.0 \mathrm{~N}$ aqueous $\mathrm{HCl}(4.0 \mathrm{~mL})$ as described above. The residue was dissolved in methanol $(7 \mathrm{~mL})$ and cooled to $0{ }^{\circ} \mathrm{C}$. To this solution was added portionwise $\mathrm{NaBH}_{4}(100 \mathrm{mg}, 2.6 \mathrm{mmol})$. The resulting mixture was warmed to room temperature and stirred for $2 \mathrm{~h}$. Aqueous $\mathrm{HCl}(3 \mathrm{~N}, 2 \mathrm{~mL})$ was slowly added. The methanol was removed under reduced pressure and the residue was extracted withj ethyl acetate $(2 \times 10 \mathrm{~mL})$. The organic layer was dried over anhydrous sodium sulfate, concentrated. The residue was purified by silica gel column chromatography (acetone:hexanes/1:4) to give $\mathbf{1 1} \mathbf{j}$ (101 $\mathrm{mg}, 64 \%$ for two steps) as a colorless oil. The ee of $\mathbf{1 1} \mathbf{j}$ was determined to be $92 \%$ [HPLC conditions: Daicel Chiralpak OJ, isopropanol:hexane $/ 10: 90,1.0 \mathrm{~mL} / \mathrm{min}, \mathrm{t}_{\mathrm{r}}($ major $)=18.7 \mathrm{~min}, \mathrm{t}_{\mathrm{r}}($ minor $)=$ $16.5 \mathrm{~min}] .[\alpha]_{\mathrm{D}}{ }^{25}=-6.9\left(\mathrm{c} 1.69, \mathrm{CHCl}_{3}\right) ;{ }^{1} \mathrm{H}$ NMR $\left(400 \mathrm{MHz}, \mathrm{CDCl}_{3}\right) \delta 1.12(\mathrm{~s}, 3 \mathrm{H})$, $2.91(\mathrm{~s}, 1 \mathrm{H}), 3.17$ (dd, $J=14.4,6.4 \mathrm{~Hz}, 1 \mathrm{H}), 3.24(\mathrm{dd}, J=14.4,6.8 \mathrm{~Hz}, 1 \mathrm{H}), 3.33-3.48$ $(\mathrm{m}, 3 \mathrm{H}), 5.11(\mathrm{~s}, 2 \mathrm{H}), 5.35-5.40(\mathrm{~m}, 1 \mathrm{H}), 7.31-7.39(\mathrm{~m}, 5 \mathrm{H}) ;{ }^{13} \mathrm{C}$ NMR $(100 \mathrm{MHz}$, $\left.\mathrm{CDCl}_{3}\right) \delta 22.16,47.63,67.36,67.48,72.97,128.34,128.53,128.79,136.26,158.26$; IR (neat) $\vee 3407,2936,1693,1537,1454 \mathrm{~cm}^{-1}$; HRMS (DCI/ $\left.\mathrm{NH}_{3}\right)$ Calcd for $\mathrm{C}_{12} \mathrm{H}_{18} \mathrm{NO}_{4}$ $\left(\mathrm{MH}^{+}\right)$240.1236, found 240.1241.

\section{Conversion of $(+)-9 j$ to to $(+)-12 j$}

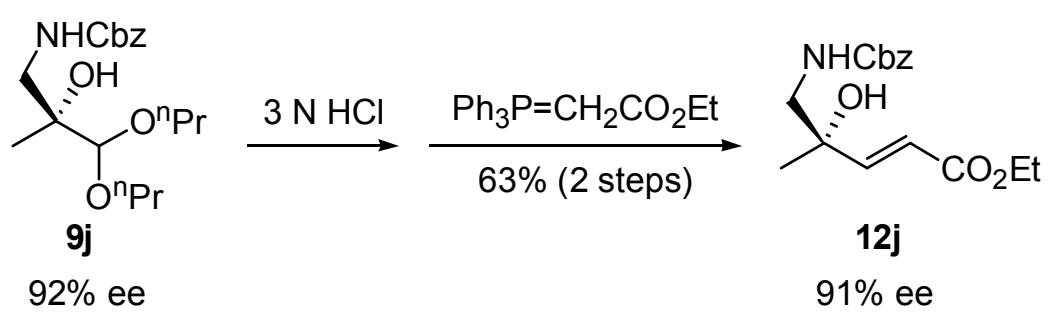

9j (225 mg, $0.66 \mathrm{mmol})$ was hydrolyzed with $3.0 \mathrm{~N}$ aqueous $\mathrm{HCl}(4.0 \mathrm{~mL})$ as described above. The residue was dissolved in benzene $(10 \mathrm{~mL})$ and $\mathrm{Ph}_{3} \mathrm{P}=\mathrm{CHCO}_{2} \mathrm{Et}$ (453 mg, $1.3 \mathrm{mmol}$ ) was added. The mixture was stirred for $2 \mathrm{~h}$ and concentrated to about $1 \mathrm{~mL}$. The residue was purified by silica gel column chromatography (ethyl acetate:hexanes/1:4) to give $\mathbf{1 2} \mathbf{j}$ (129 $\mathrm{mg}, \mathbf{6 3} \%$ for two steps) as a colorless oil. The ee of 
12j was determined to be 91\% [HPLC conditions: Daicel Chiralpak OJ, isopropanol:hexane $/ 10: 90,1.0 \mathrm{~mL} / \mathrm{min}, \mathrm{t}_{\mathrm{r}}$ (major) $=28.5 \mathrm{~min}, \mathrm{t}_{\mathrm{r}}$ (minor) $\left.=38.4 \mathrm{~min}\right]$. $[\alpha]_{\mathrm{D}}{ }^{25}=22.2\left(\mathrm{c} 1.95, \mathrm{CHCl}_{3}\right) ;{ }^{1} \mathrm{H}$ NMR $\left(400 \mathrm{MHz}, \mathrm{CDCl}_{3}\right) \delta 1.29(\mathrm{t}, J=7.0 \mathrm{~Hz}, 3 \mathrm{H})$, 1.33 (s, 3H), 2.96 (s, $1 \mathrm{H}), 3.24$ (dd, $J=14.0,6.0 \mathrm{~Hz}, 1 \mathrm{H}), 3.40$ (dd, $J=14.0,6.8 \mathrm{~Hz}, 1 \mathrm{H}$ ), 4.19 (q, $J=7.0 \mathrm{~Hz}, 2 \mathrm{H}), 5.10(\mathrm{~s}, 2 \mathrm{H}), 5.11-5.16(\mathrm{~m}, 1 \mathrm{H}), 6.13(\mathrm{~d}, J=15.6 \mathrm{~Hz}, 1 \mathrm{H}), 6.88$ (d, $J=15.6 \mathrm{~Hz}, 1 \mathrm{H}), 7.31-7.39(\mathrm{~m}, 5 \mathrm{H}) ;{ }^{13} \mathrm{C} \mathrm{NMR}\left(100 \mathrm{MHz}, \mathrm{CDCl}_{3}\right) \delta 14.42,25.69$, $50.51,60.78,67.42,73.73,121.14,128.32,128.47,128.78,136.34,151.21,157.87$, 166.65; IR (neat) $\vee 3371,2962,1713,1644,1537,1455 \mathrm{~cm}^{-1}$; HRMS (DCI/NH $\mathrm{NH}_{3}$ Calcd for $\mathrm{C}_{16} \mathrm{H}_{22} \mathrm{NO}_{5}\left(\mathrm{MH}^{+}\right)$308.1498, found 308.1485.

\section{Preparation of $\beta$-hydroxylamine 12d from silylcyanohydrin 8d:}

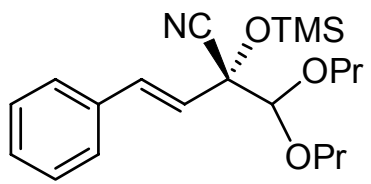

8d $(91 \%$ ee $)$ (i) $\mathrm{LiAlH}_{4}, \mathrm{THF} / \mathrm{Et}{ }_{2} \mathrm{O}$, rt. 3h

(ii) $\mathrm{CbzCl}, \mathrm{TEA}, \mathrm{Et}_{2} \mathrm{O}$, rt. $90 \%$

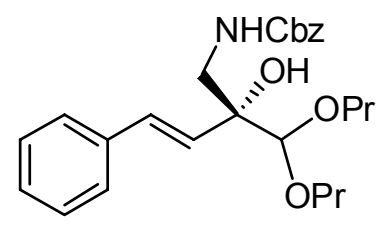

13d $(90 \%$ ee $)$

To a suspension of $\mathrm{LiAlH}_{4}(13 \mathrm{mg}, 0.346 \mathrm{mmol}, 5.0$ eq.) in dry THF $(0.5 \mathrm{~mL})$ was added dry diethyl ether $(2.5 \mathrm{~mL})$. The mixture was stirred at room temperature for 30 $\mathrm{min}$, and then to this mixture was added dropwise a solution of $8 \mathbf{d}(25 \mathrm{mg}, 0.87 \mathrm{mmol})$ in dry ether $(2.0 \mathrm{~mL})$ under $\mathrm{N}_{2}$ at room temperature. The mixture was stirred for $30 \mathrm{~min}$, and then water $(0.5 \mathrm{~mL})$ was added dropwise to the mixture at $0^{\circ} \mathrm{C}$ via a syringe. The resulting mixture was stirred at room temperature for an additional $30 \mathrm{~min}$. Then anhydrous sodium sulfate $(300 \mathrm{mg})$ was added in one portion to this mixture. The mixture was vigorously stirred at room temperature for $1 \mathrm{~h}$, then filtered and washed with ether. The combined ether solution was concentrated and used without further purification.

The crude $\beta$-amino alcohol was dissolved in dry ether $(4.0 \mathrm{~mL})$. Benzyl chloroformate ( $12 \mu \mathrm{L}, 14 \mathrm{mg}, 0.084 \mathrm{mmol}, 1.2$ eq.). Triethylamine ( $15 \mu \mathrm{L}, 11 \mathrm{mg}, 0.108$ mmol, 1.5 eq.) were added dropwise at room temperature via a pipette. The resulting white suspension was stirred at room temperature for $1 \mathrm{~h}$, then filtered through a short pad of silica gel. The silica gel pad was washed with ether $(3 \times 10 \mathrm{~mL})$. The combined ether solution was concentrated and the residue was subjected to silica gel chromatography (Hexanes: EtOAc $=3: 1)$ to afford the desired product 13d (27 mg, 90\% yield). The ee of 13d was determined to be $90 \%$. $[\alpha]_{\mathrm{D}}{ }^{25}=-33.4\left(\mathrm{c} 0.70, \mathrm{CHCl}_{3}\right)$. HPLC analysis conditions: Chiralcel OJ, isopropanol:hexanes $/ 15: 85,0.5 \mathrm{~mL} / \mathrm{min}, \lambda=254 \mathrm{~nm} . \mathrm{t}_{\mathrm{r}}$ (minor) $=1.6 \mathrm{~min}, \mathrm{t}_{\mathrm{r}}$ (major) $=16.2 \mathrm{~min} .{ }^{1} \mathrm{H}$ NMR $\left(400 \mathrm{MHz}, \mathrm{CDCl}_{3}\right): \delta 0.95,0.94($ each $\mathrm{t}, \mathrm{J}=$ 7.2 Hz, $\left.\mathrm{CH}_{3}\right), 1.57-1.70\left(\mathrm{~m}, 4 \mathrm{H}, 2 \times \mathrm{CH}_{2}\right), 2.81(\mathrm{~s}, 1 \mathrm{H}, \mathrm{OH}), 3.42-3.55\left(\mathrm{~m}, 4 \mathrm{H}, 2 \times \mathrm{CH}_{2} \mathrm{O}\right)$, $3.75\left(\mathrm{M}, 2 \mathrm{H}, \mathrm{CH}_{2}-\mathrm{NH}\right), 4.34(\mathrm{~s}, 1 \mathrm{H},-\mathrm{CH}), 5.06\left(\mathrm{~s}, 2 \mathrm{H}, \mathrm{OCH}_{2} \mathrm{Ph}\right), 5.16$ (brs, $\left.1 \mathrm{H}, \mathrm{NH}\right)$, $6.29(\mathrm{~d}, \mathrm{~J}=16 \mathrm{~Hz}, 1 \mathrm{H},-\mathrm{CH}=), 6.78(\mathrm{~d}, \mathrm{~J}=16 \mathrm{~Hz}, 1 \mathrm{H}, \mathrm{PhCH}=)$, 7.25-7.39 (m, $10 \mathrm{H}, \mathrm{Ar}-$ H). ${ }^{13} \mathrm{C}$ NMR $\left(100 \mathrm{MHz}, \mathrm{CDCl}_{3}\right): \delta 10.60,23.14,23.21,45.58,66.65,72.20,72.69$, 77.20, 106.97, 126.61, 127.53, 127.92, 127.95, 128.41, 128.49, 128.53, 130.87, 136.74, 157.11. FT-IR (neat) $\vee 1721 \mathrm{~cm}^{-1}$. HRMS: Calcd. for $\mathrm{C}_{25} \mathrm{H}_{33} \mathrm{NO}_{5} \mathrm{Na}$ : 450.2256; Found: 450.2268 . 
<smiles>CCCOC(OCC)[C@@](O)(C=Cc1ccccc1)CNC(=O)OCC</smiles>

13d (90\%еe) (i) $3 \mathrm{~N} \mathrm{HCl}$, acetone, $30 \mathrm{~h}$

(ii) (EtO) ${ }_{2} \mathrm{OPCH}_{2} \mathrm{CO}_{2} \mathrm{Et}$

$\mathrm{NaH}(60 \%$ assay), THF, rt.

$73 \%$

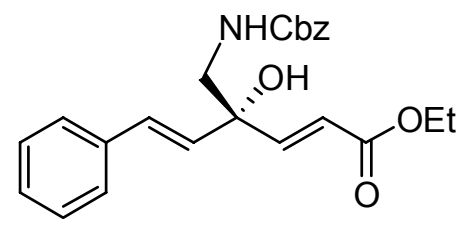

12d (90\%ee)

To a flask charged with a solution of $\mathbf{1 3 d}(15 \mathrm{mg}, 0.035 \mathrm{mmol})$ in acetone $(3.0 \mathrm{~mL})$ was added $\mathrm{HCl}(3.0 \mathrm{~N}, 1.0 \mathrm{~mL})$ at room temperature. The flask was sealed by a septum. The mixture was stirred at room temperature for $30 \mathrm{~h}$, and then aqueous potassium carbonate $(20 \mathrm{wt} \%)$ was added to the mixture to adjust the $\mathrm{pH}$ value to 9-11. The resulting mixture was extracted with diethyl ether $(3 \times 5 \mathrm{~mL})$. The combined organic layers were washed with brine, dried over anhydrous sodium sulfate, and concentrated in vacuo. The crude aldehyde was subjected to the next step without further purification.

An oven-dried Schlenk tube $(5.0 \mathrm{~mL})$ was charged under nitrogen with a stir bar, sodium hydride ( $4.2 \mathrm{mg}, 0.105 \mathrm{mmol}, 3$ eq.) and distilled THF $(1.0 \mathrm{~mL})$. Triethyl phosphonoacetate $(28 \mu \mathrm{L}, 31 \mathrm{mg}, 0.14 \mathrm{mmol}, 4$ eq.) was added dropwise via a pipette. The mixture was stirred at room temperature for 30 min until no gas was emitted. A solution of the crude aldehyde in THF $(1.0 \mathrm{~mL})$ was added dropwise via a syringe at $0^{\circ} \mathrm{C}$. The mixture turned to red immediately upon the addition of the aldehyde. The color faded to yellow after the mixture was stirred for $30 \mathrm{~min}$. The mixture was stirred at room temperature for an additional $30 \mathrm{~min}$ and then concentrated. The residue was subjected to preparative TLC plate $\left(\mathrm{CH}_{2} \mathrm{Cl}_{2} / \mathrm{MeOH}=50 / 1\right)$ to give the desired $(E)$-isomer 12d in (10 $\mathrm{mg}, 73 \%$ yield). The ee of $\mathbf{1 2 d}$ was determined to be $90 \%$. $[\alpha]_{\mathrm{D}}{ }^{25}=7.8$ (c 0.50 , $\mathrm{CHCl}_{3}$ ). HPLC analysis conditions: Chiralcel OD, isopropanol:hexanes/10:90, 1.0 $\mathrm{mL} / \mathrm{min}, \lambda=254 \mathrm{~nm} . \mathrm{t}_{\mathrm{r}}$ (major) $=41.8 \mathrm{~min}, \mathrm{t}_{\mathrm{r}}($ minor $)=48.1 \mathrm{~min} .{ }^{1} \mathrm{H} \mathrm{NMR}(400 \mathrm{MHz}$, $\left.\mathrm{CDCl}_{3}\right): \delta 1.29\left(\mathrm{t}, \mathrm{J}=6.8 \mathrm{~Hz}, \mathrm{CH}_{3}\right), 3.35(\mathrm{~s}, 1 \mathrm{H}, \mathrm{OH}), 3.42(\mathrm{dd}, \mathrm{J}=6,14 \mathrm{~Hz}, 1 \mathrm{H}, \mathrm{AB},-$ $\left.\mathrm{CH}_{2}-\mathrm{NH}\right), 3.51$ (dd, J = 6, $\left.14 \mathrm{~Hz}, 1 \mathrm{H}, \mathrm{AB},-\mathrm{CH}_{2}-\mathrm{NH}\right), 4.20\left(\mathrm{q}, \mathrm{J}=6.8 \mathrm{~Hz}, 2 \mathrm{H},-\mathrm{O}-\mathrm{CH}_{2}-\right.$ $\left.\mathrm{CH}_{3}\right), 5.09\left(\mathrm{~s}, 2 \mathrm{H}, \mathrm{OCH}_{2} \mathrm{Ph}\right), 5.16(\mathrm{t}, \mathrm{J}=6 \mathrm{~Hz}, 1 \mathrm{H}, \mathrm{NH}), 6.19(\mathrm{~d}, \mathrm{~J}=16 \mathrm{~Hz},=\mathrm{CH}-), 6.22$ $(\mathrm{d}, \mathrm{J}=16 \mathrm{~Hz}, 1 \mathrm{H},=\mathrm{CHCO}), 6.74(\mathrm{~d}, \mathrm{~J}=16 \mathrm{~Hz}, 1 \mathrm{H},-\mathrm{CH}=), 6.98(\mathrm{~d}, \mathrm{~J}=15.2 \mathrm{~Hz}, 1 \mathrm{H}$, $\mathrm{PhCH}=)$, 7.25-7.37 (m, $10 \mathrm{H}, \mathrm{Ar}-\mathrm{H}) .{ }^{13} \mathrm{C}$ NMR $\left(100 \mathrm{MHz}, \mathrm{CDCl}_{3}\right): \delta 14.44,49.86,60.88$, 67.50, 76.74, 121.59, 126.03, 126.91, 128.30, 128.33, 128.46, 128.77, 128.87, 129.52, 131.32, 136.28, 148.74, 158.05, 166.57. FT-IR (neat) $v 1712 \mathrm{~cm}^{-1}$. HRMS: Calcd. for $\mathrm{C}_{23} \mathrm{H}_{29} \mathrm{~N}_{2} \mathrm{O}_{5}$ : 413.2076; found: 413.2079 . 
HPLC conditions: Daicel Chiralpak OD, isopropanol:hexane $/ 15: 85,1.0 \mathrm{~mL} / \mathrm{min}, \lambda=254$ $\mathrm{nm}$
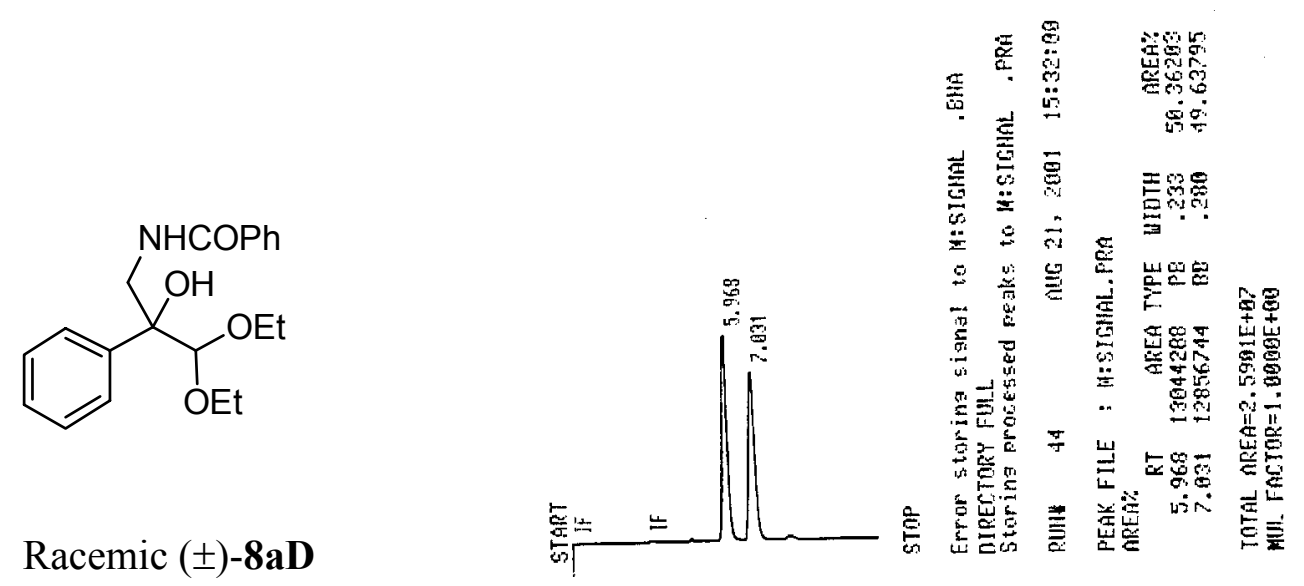

Derivative of the product $(+)-\mathbf{8 a}$ of $(\mathrm{DHQ})_{2} \mathrm{AQN}-$ catalyzed reaction

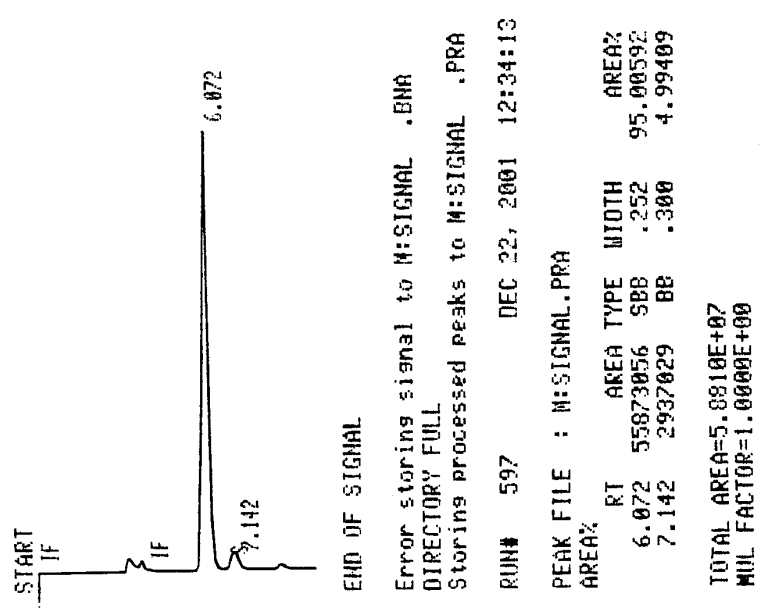


HPLC conditions: Daicel Chiralpak AD, isopropanol:hexane/0.2:99.8, $0.20 \mathrm{~mL} / \mathrm{min}, \lambda=$ $220 \mathrm{~nm}$<smiles>CCOC(OCC)C(C#N)(O[Na])c1ccc(OC)cc1</smiles>

\section{Racemic ( \pm$)-8 b$}

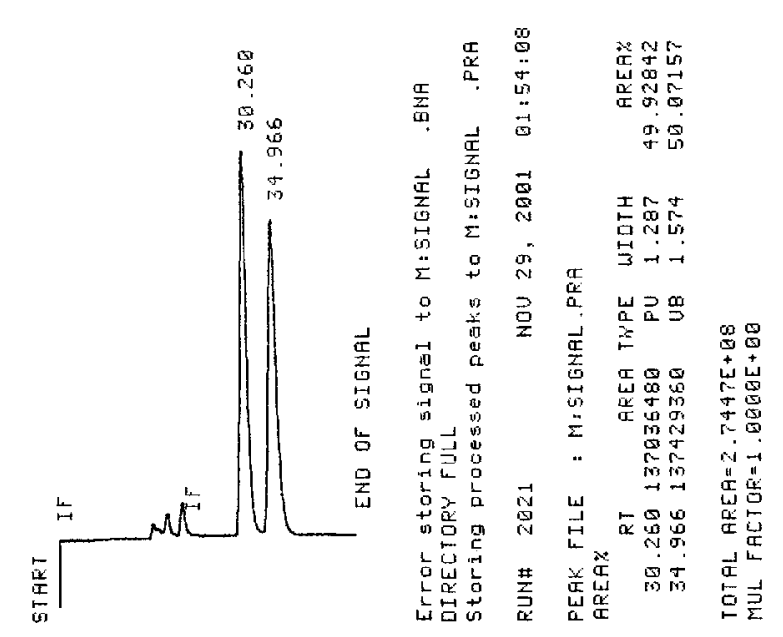

Product $(+)-\mathbf{8 b}$ of (DHQ) ${ }_{2} \mathrm{AQN}-$ catalyzed reaction

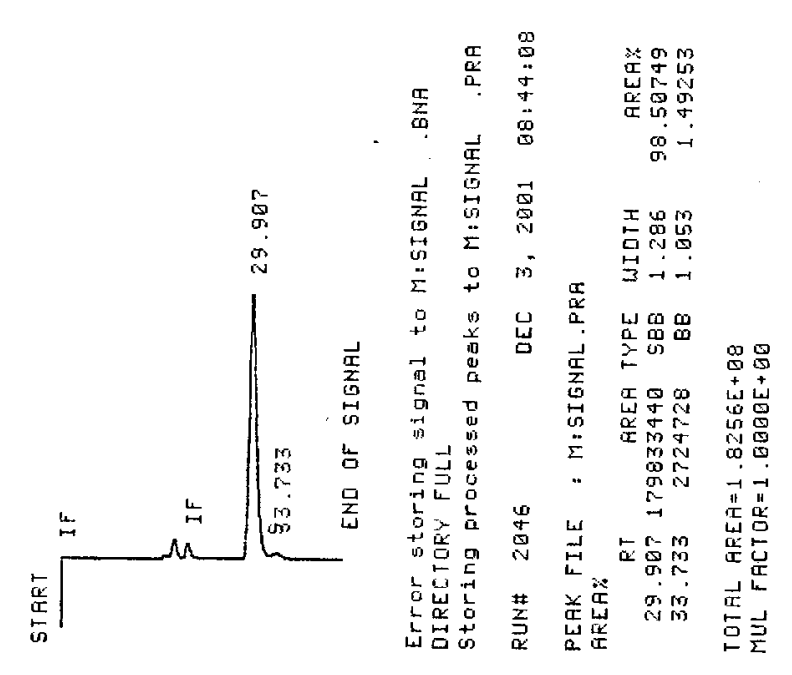


GC conditions: Gamma cyclodextrin Trifluoroacetyl, $115^{\circ} \mathrm{C}, 4.0 \mathrm{~min}, 0.05^{\circ} \mathrm{C} / \mathrm{min}$ to 122 ${ }^{\circ} \mathrm{C}$

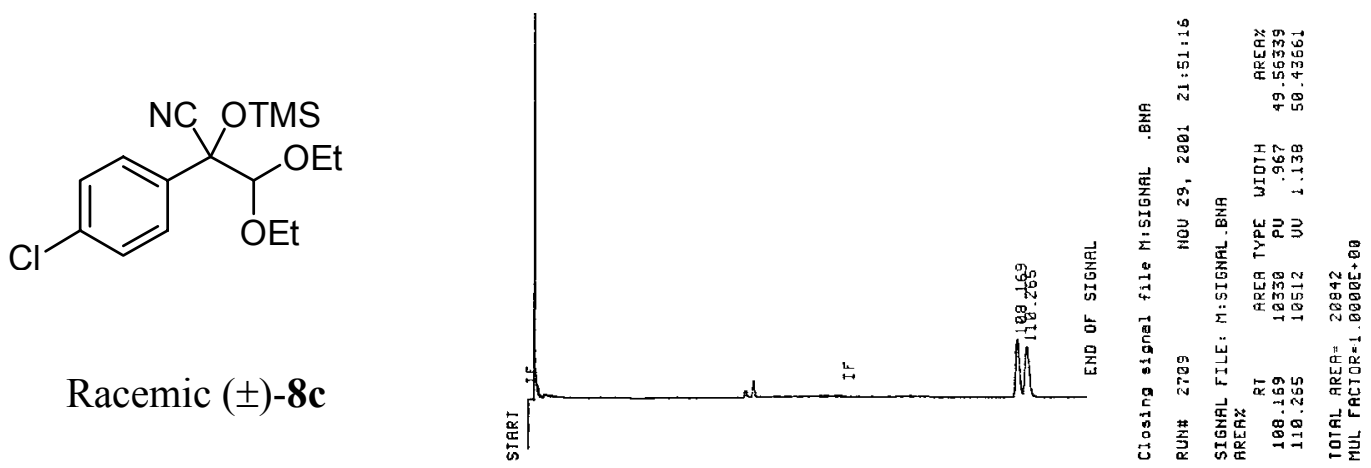

Product $(+)-8 \mathbf{c}$ of $(\mathrm{DHQ})_{2} \mathrm{AQN}-$ catalyzed reaction

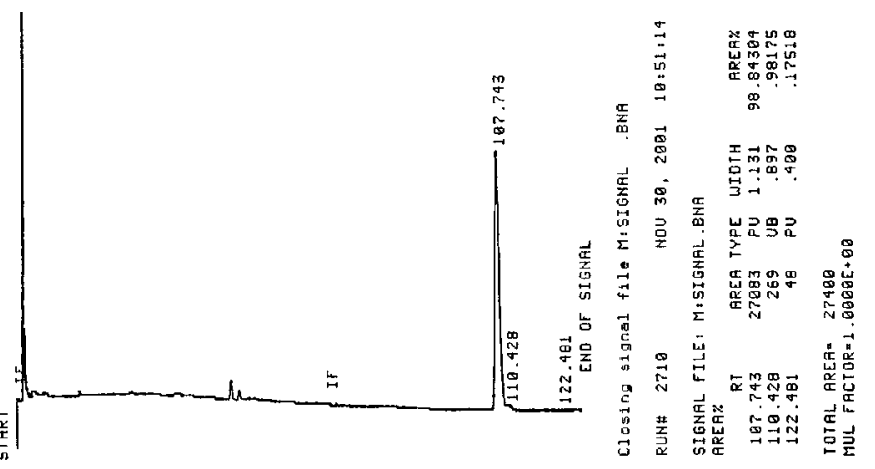

Product (-)-8c of (DHQD) 2 PHALcatalyzed reaction

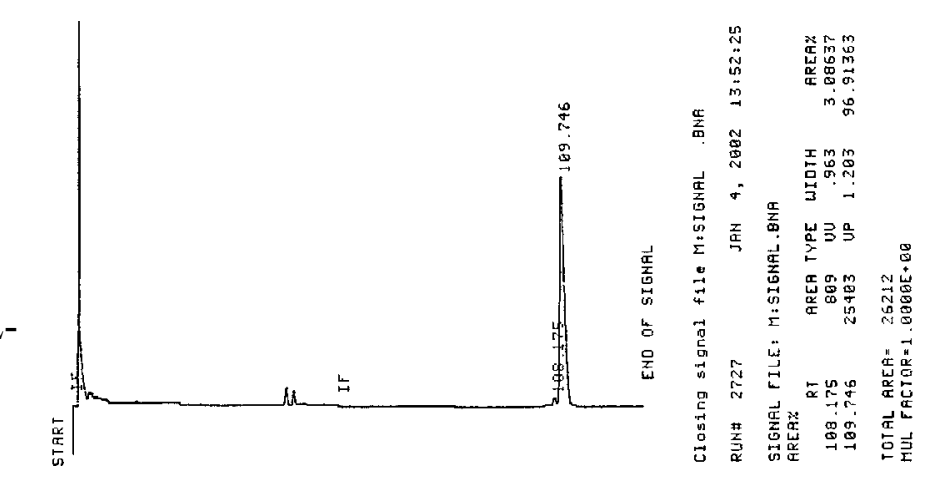


HPLC conditions: Daicel Chiralpak AD, isopropanol:hexane $/ 1: 99,0.10 \mathrm{~mL} / \mathrm{min}, \lambda=254$ $\mathrm{nm}$

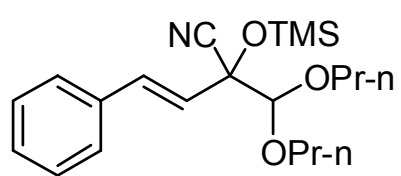

Racemic ( \pm$)-8 d$

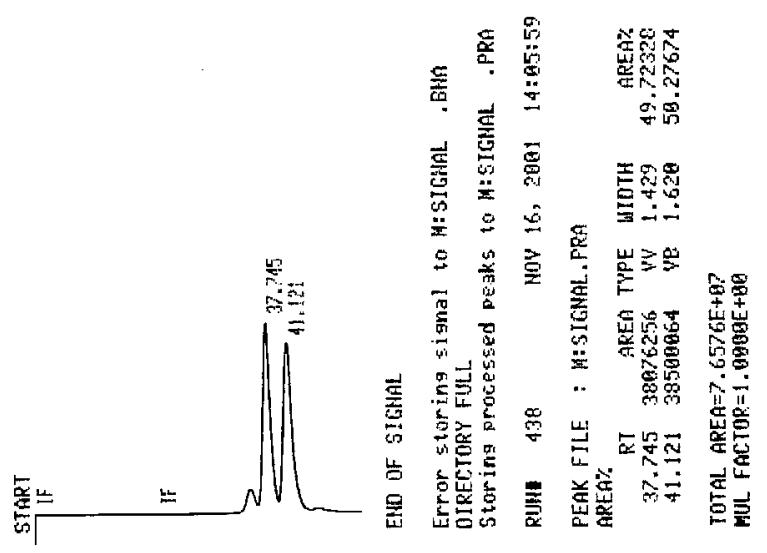

Product $(+)-8 d$ of $(\mathrm{DHQ})_{2} \mathrm{AQN}-$ catalyzed reaction

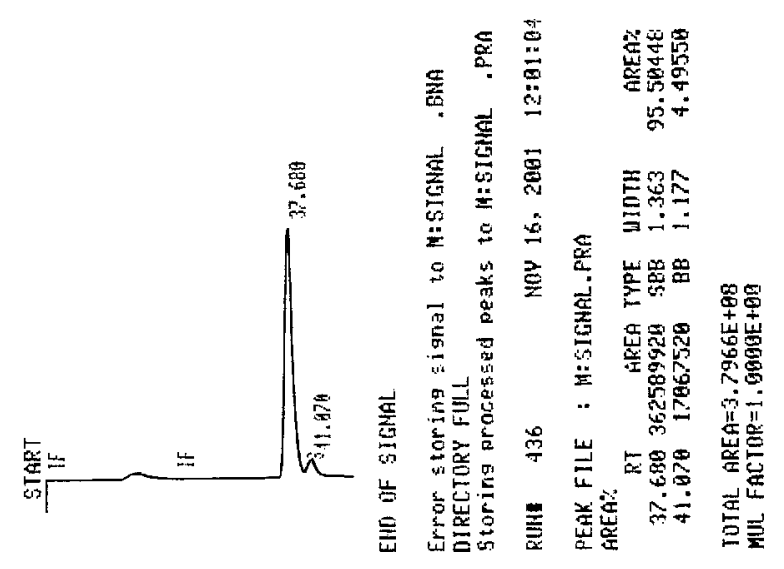


HPLC conditions: Daicel Chiralpak AD, isopropanol:hexane/1:99, $0.20 \mathrm{~mL} / \mathrm{min}, \lambda=254$ $\mathrm{nm}$

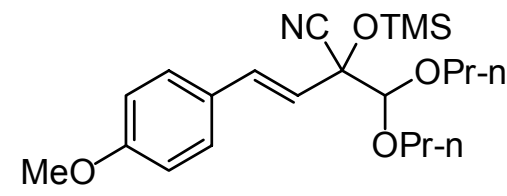

Racemic $( \pm)-8 \mathbf{e}$

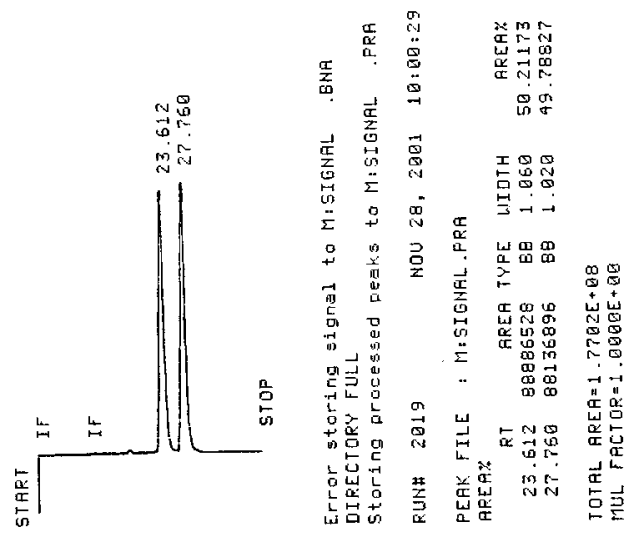

Product (+)-8e of (DHQ) ${ }_{2} \mathrm{AQN}$-catalyzed reaction

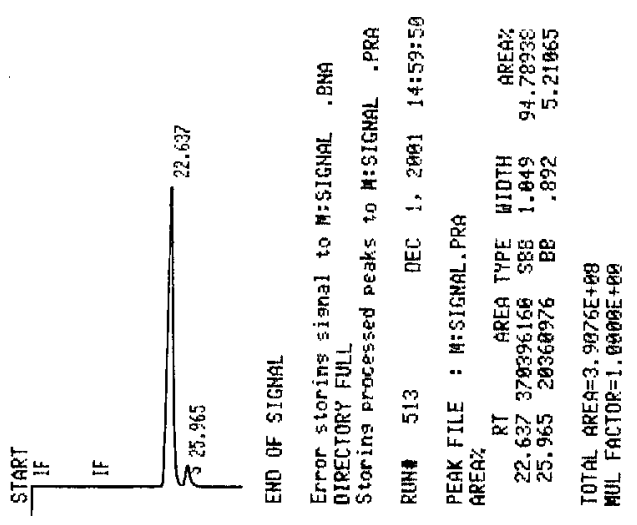

Product (-)-8e of (DHQD) $)_{2}$ PHALcatalyzed reaction

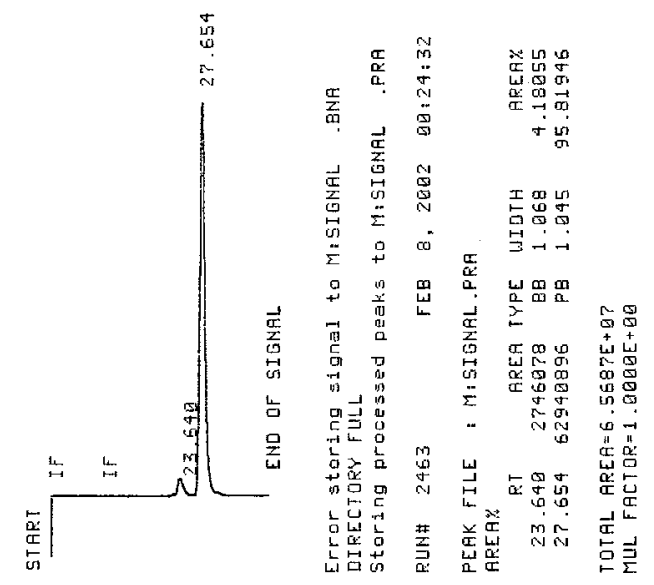


HPLC conditions: Daicel Chiralpak AD, isopropanol:hexane $/ 0.2: 99.8,1.0 \mathrm{~mL} / \mathrm{min}, \lambda=$ $254 \mathrm{~nm}$

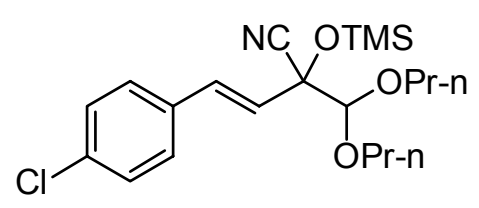

Racemic ( \pm -)-8f

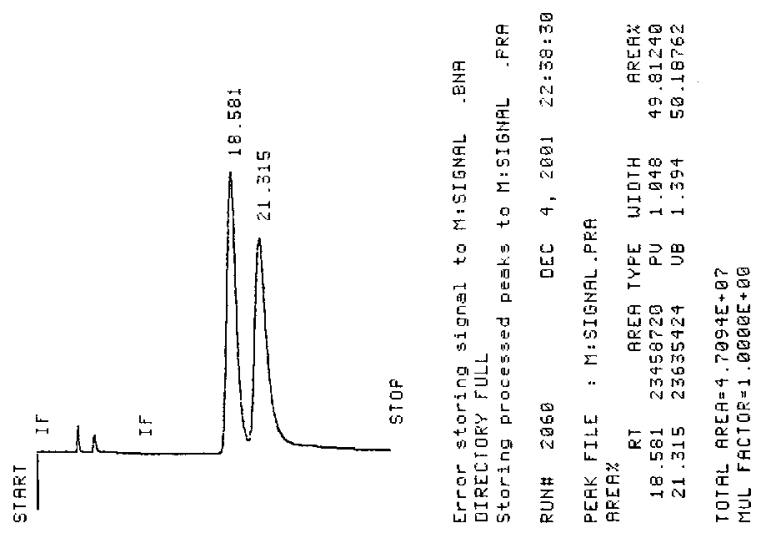

Product $(+)-8 \mathbf{f}$ of $(\mathrm{DHQ})_{2} \mathrm{AQN}-$ catalyzed reaction

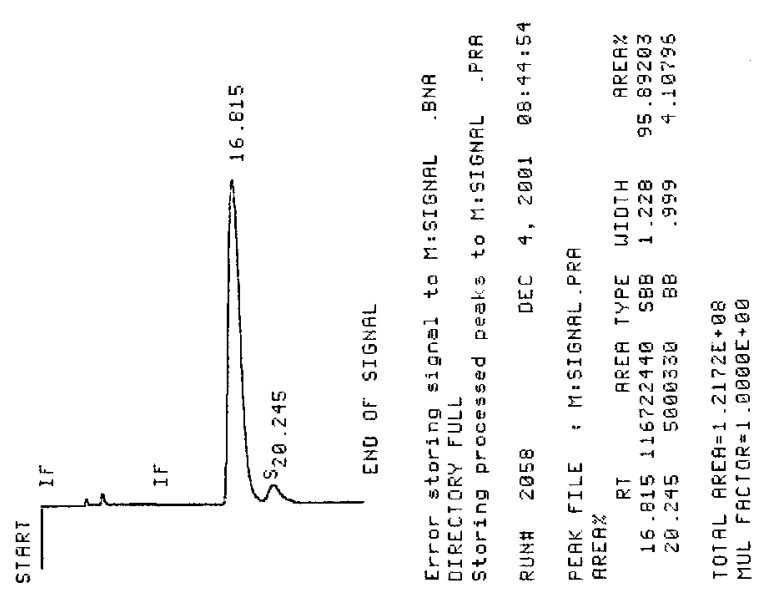


HPLC conditions: Daicel Chiralpak AD, isopropanol:hexane $/ 1: 99,0.5 \mathrm{~mL} / \mathrm{min}, \lambda=254$ $\mathrm{nm}$

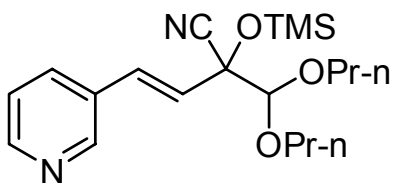

\section{Racemic ( \pm$)-8 g$}

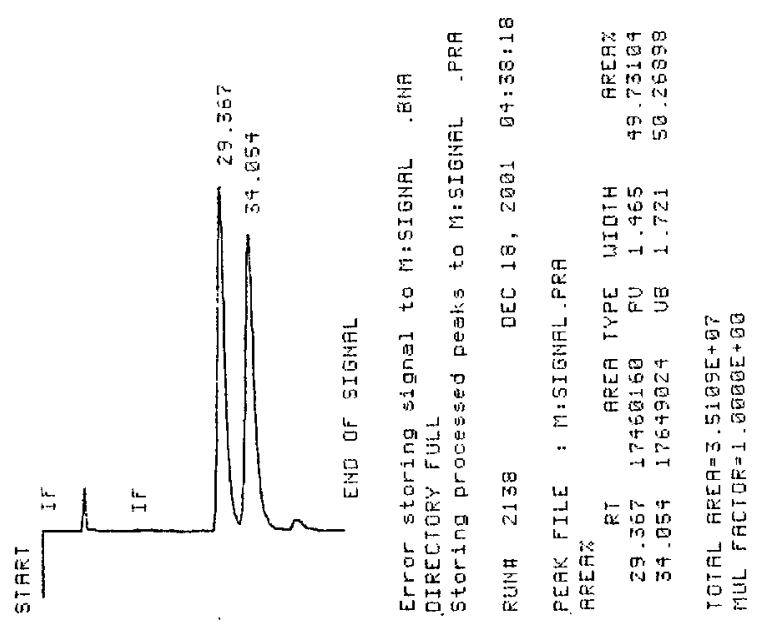

Product $(+)-8 g$ of (DHQ) ${ }_{2} \mathrm{AQN}$-catalyzed reaction

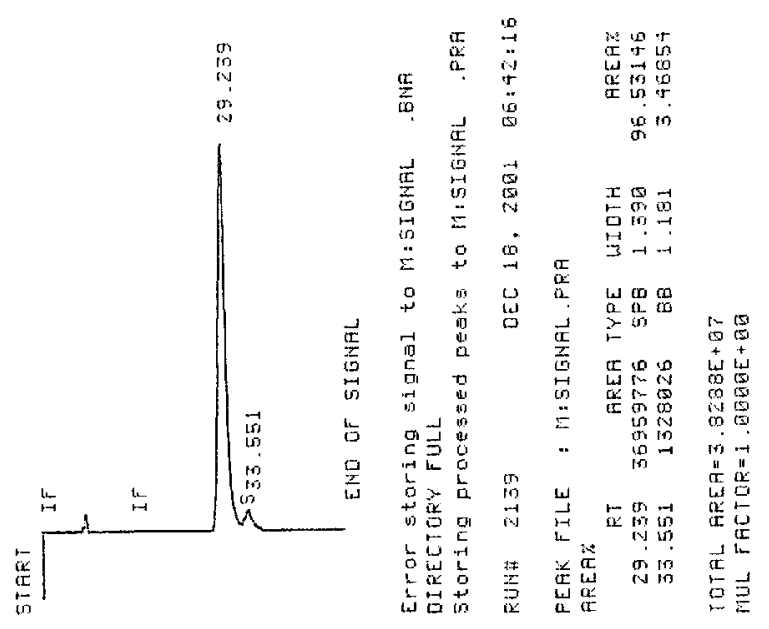


HPLC conditions: Hypersil SI + Daicel Chiralpak AS, isopropanol:hexane/1:99, 0.20 $\mathrm{mL} / \mathrm{min}, \lambda=254 \mathrm{~nm}$

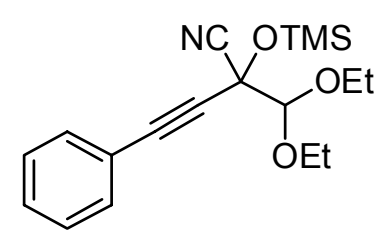

Racemic ( \pm )-8h
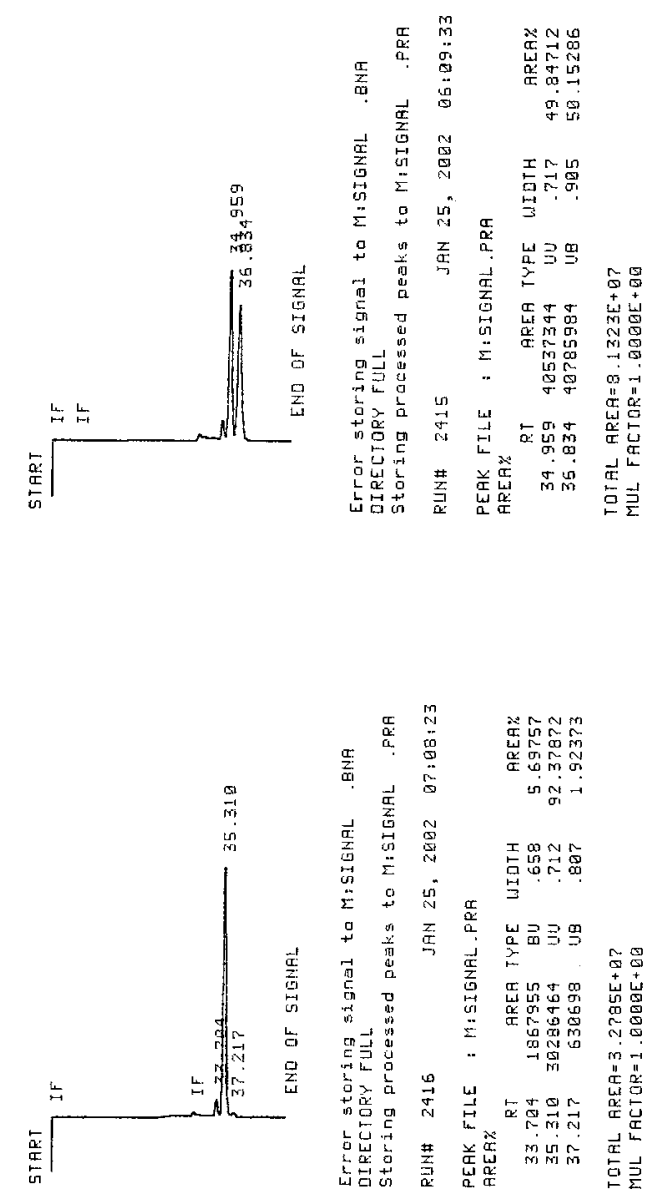

Product $(+)-\mathbf{8 h}$ of $(\mathrm{DHQ})_{2} \mathrm{AQN}-$ catalyzed reaction

Product (-)-8h of (DHQD) $)_{2}$ PHALcatalyzed reaction

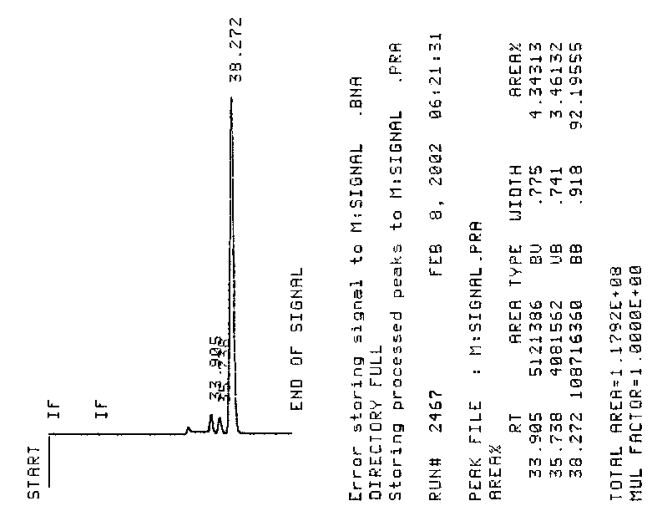


HPLC conditions: Daicel Chiralpak AD, isopropanol:hexane $/ 5: 95,1.0 \mathrm{~mL} / \mathrm{min}, \lambda=254$ $\mathrm{nm}$
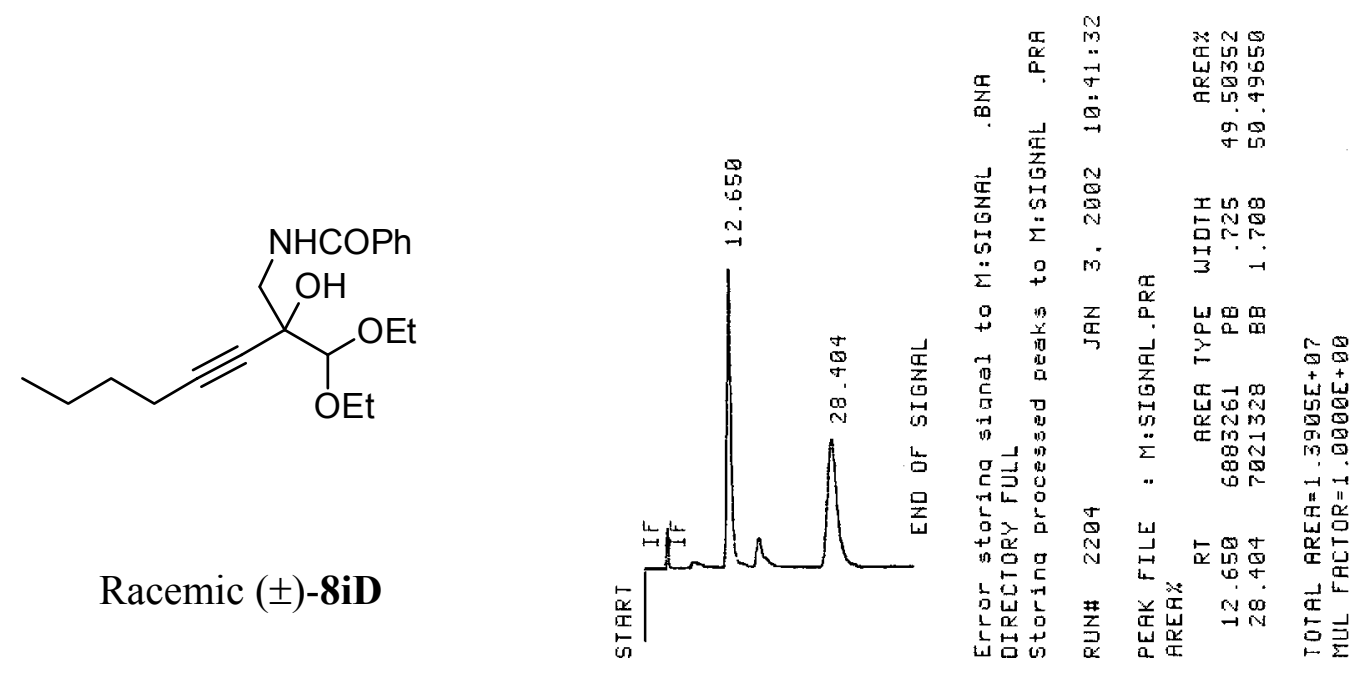

\section{Racemic ( \pm -8iD}

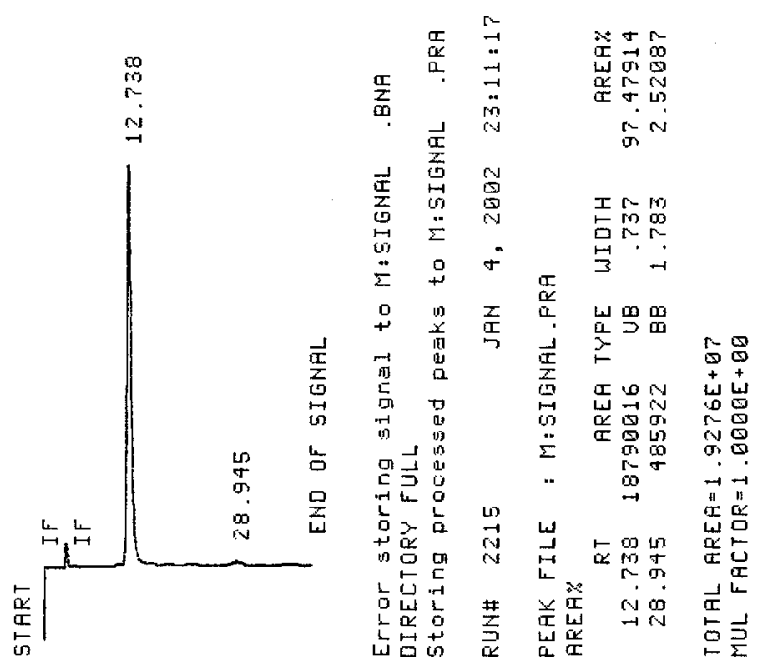


GC conditions: Gamma cyclodextrin Trifluoroacetyl, $95{ }^{\circ} \mathrm{C}, 4.0 \mathrm{~min}, 0.10{ }^{\circ} \mathrm{C} / \mathrm{min}$ to 98 ${ }^{\circ} \mathrm{C}$
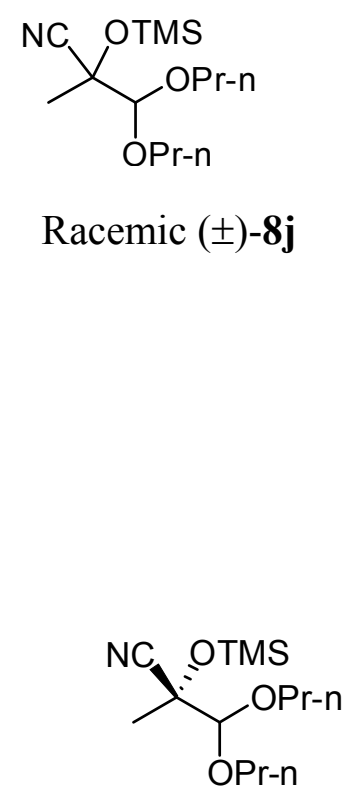

$R-(-)-8 \mathbf{j}$

Product of $(\mathrm{DHQ})_{2} \mathrm{AQN}$-catalyzed reaction

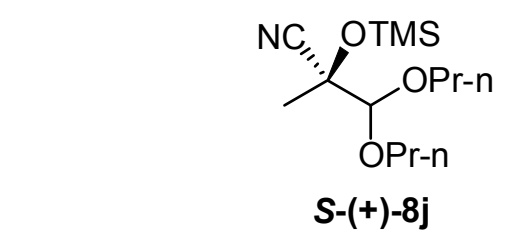

Product of $(\mathrm{DHQD})_{2}$ PHAL-catalyzed
reaction

Product of $(\mathrm{DHQD})_{2}$ PHAL-catalyzed
reaction
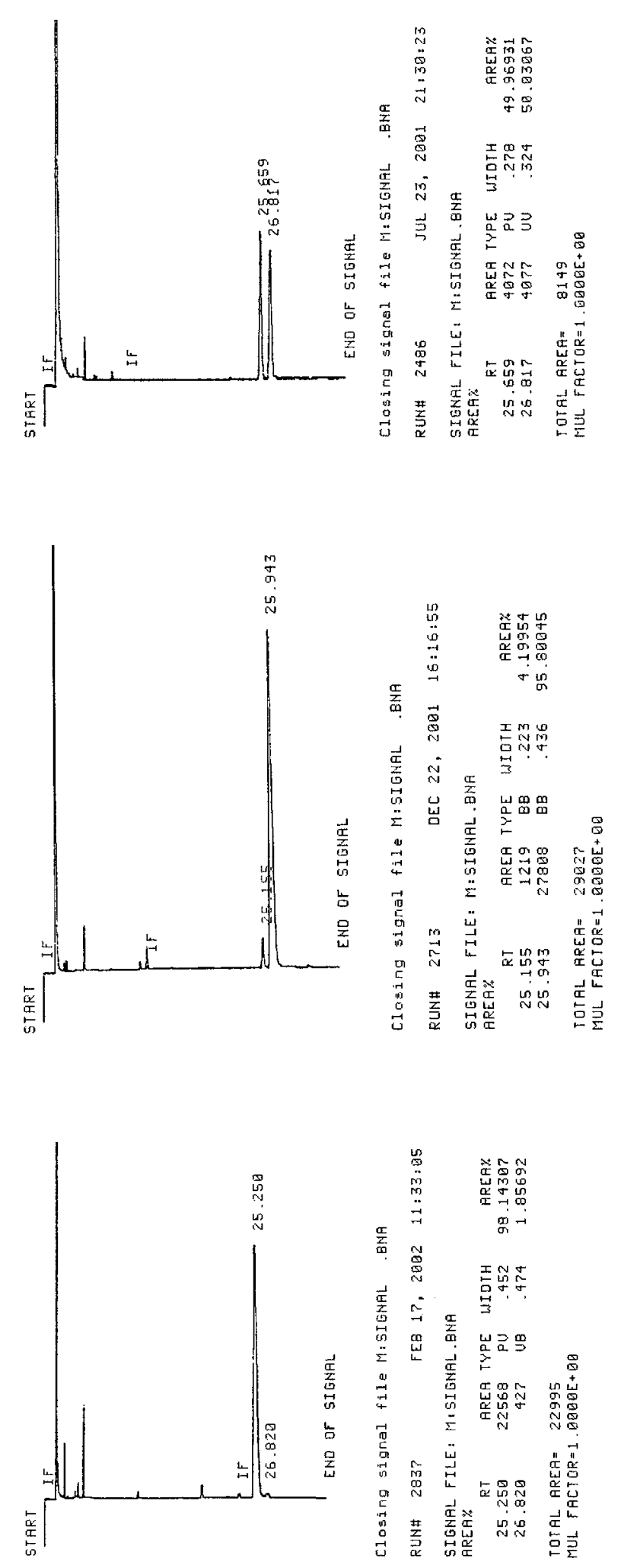
HPLC conditions: Daicel Chiralpak AD, isopropanol:hexane $/ 15: 85,1.0 \mathrm{~mL} / \mathrm{min}, \lambda=254$ $\mathrm{nm}$

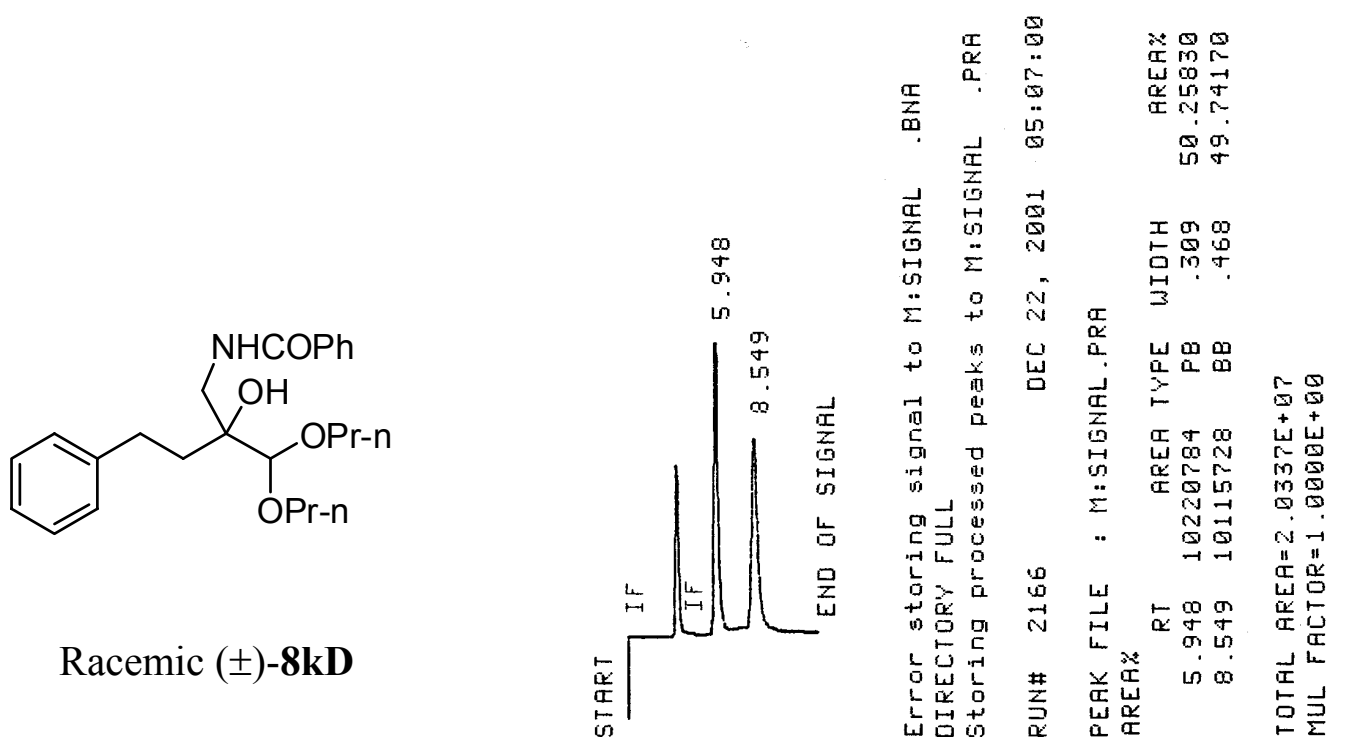

Derivative of the product $(+)-\mathbf{8 k}$ of $(\mathrm{DHQ})_{2} \mathrm{AQN}-$ catalyzed reaction

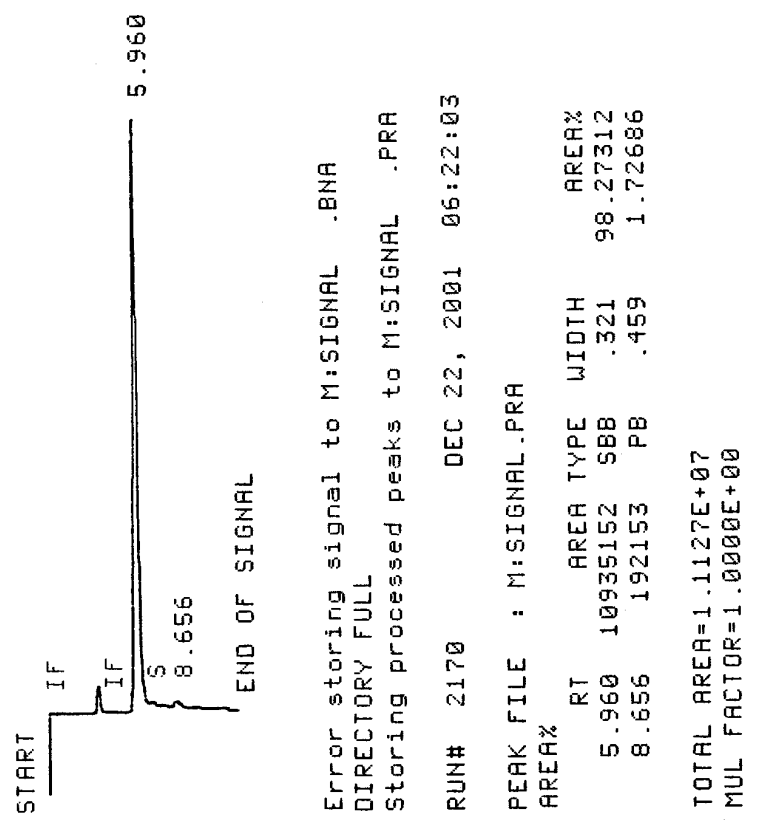


HPLC conditions: Daicel Chiralpak AD, isopropanol:hexane $/ 5: 95,1.0 \mathrm{~mL} / \mathrm{min}, \lambda=254$ $\mathrm{nm}$

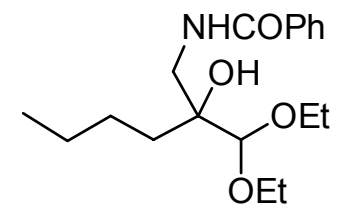

Racemic ( \pm )-8ID

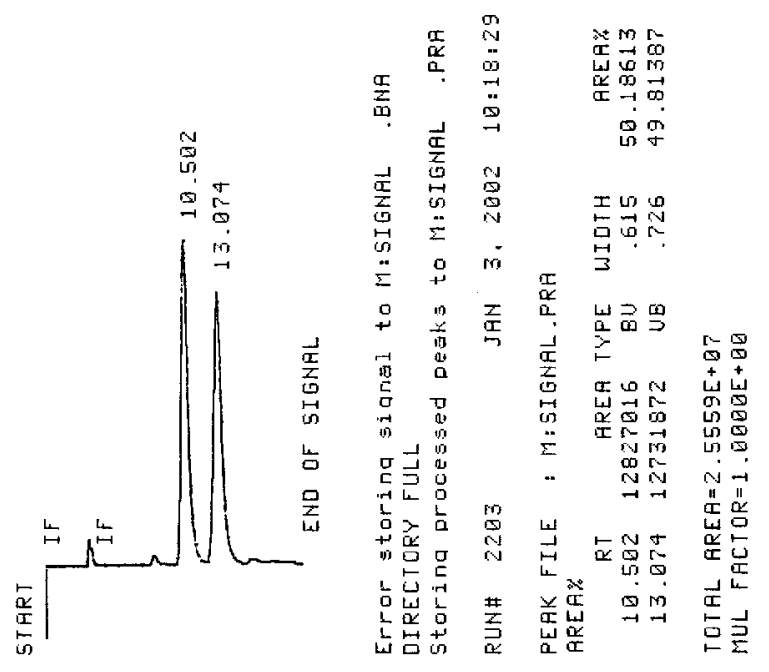

Derivative of the product $(+)-\mathbf{8} \mathbf{l}$ of $(\mathrm{DHQ})_{2} \mathrm{AQN}$-catalyzed reaction

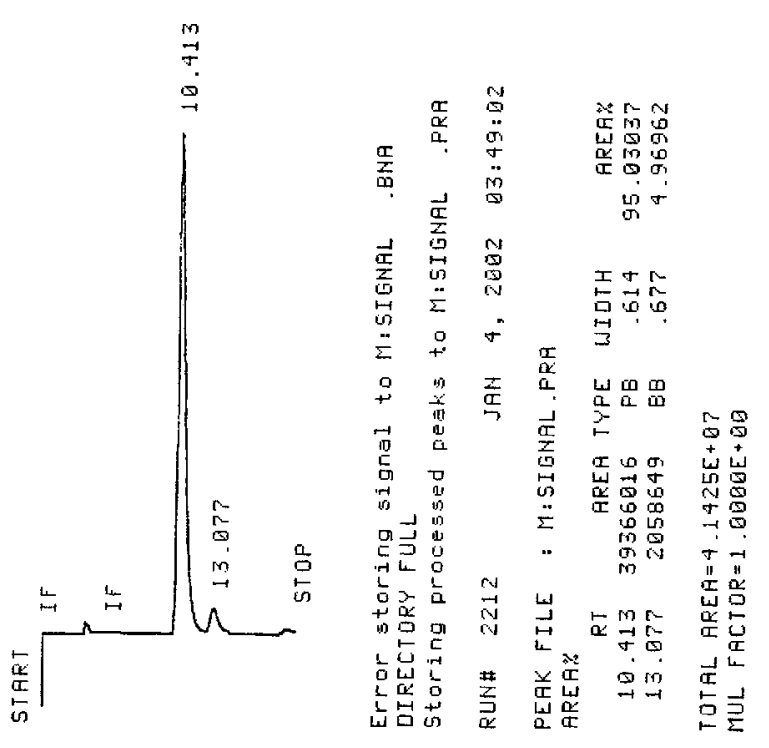


HPLC conditions: Daicel Chiralpak OD + Hypersil SI, isopropanol:hexane/2:98, 1.0 $\mathrm{mL} / \mathrm{min}, \lambda=254 \mathrm{~nm}$

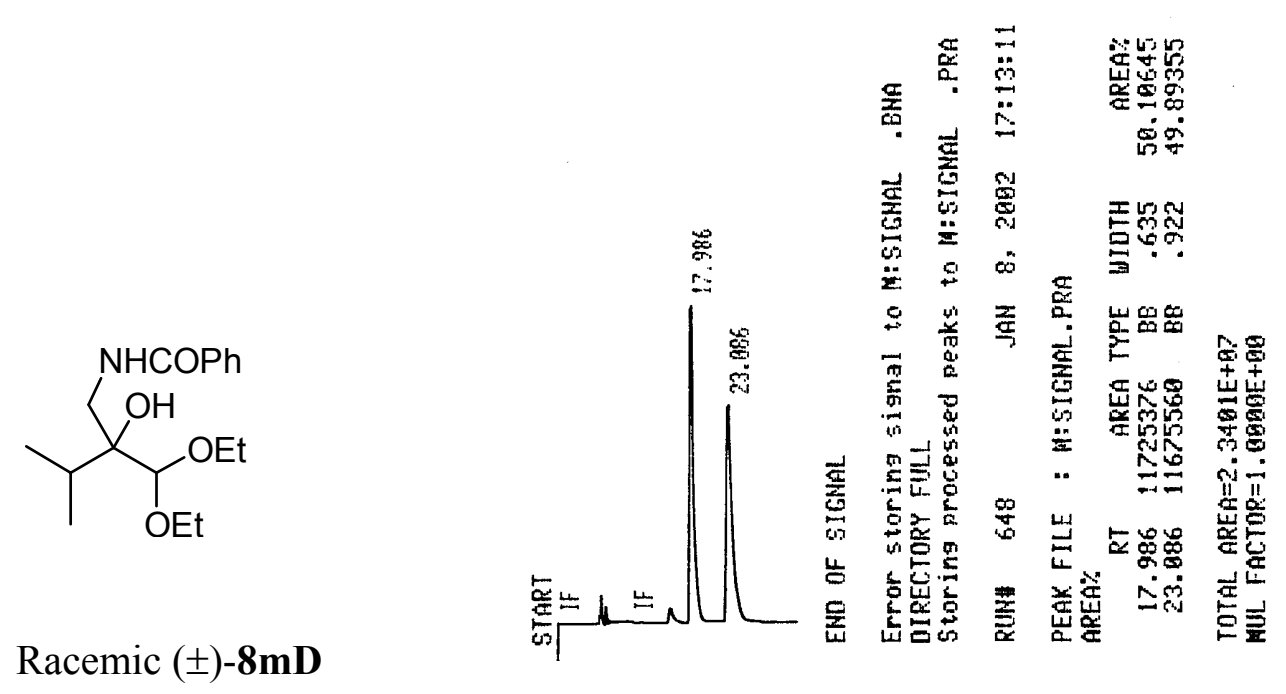

Derivative of the product $(+)-8 m$ of $(\mathrm{DHQ})_{2} \mathrm{AQN}$-catalyzed reaction

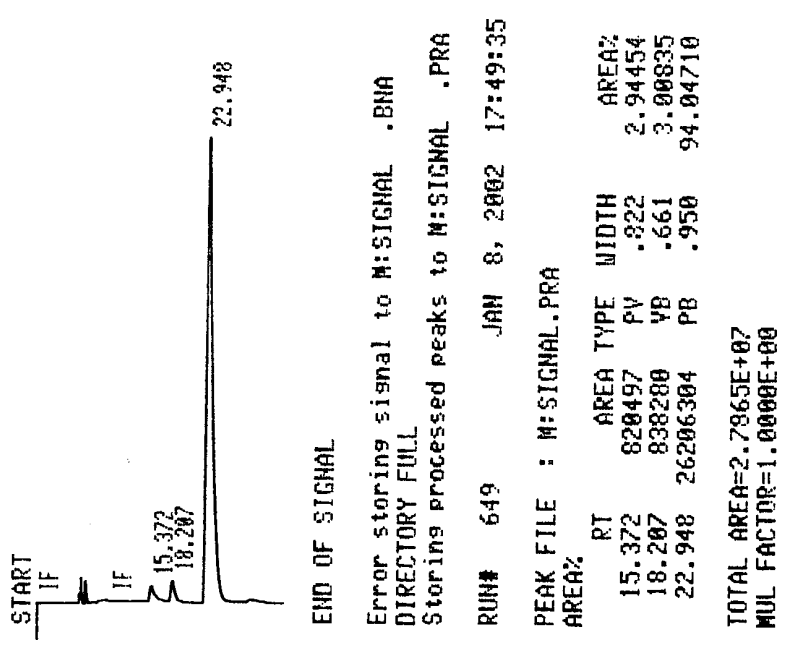


HPLC conditions: Daicel Chiralcel OJ, isopropanol:hexane/15:85, $0.5 \mathrm{~mL} / \mathrm{min}, \lambda=254$ $\mathrm{nm}$
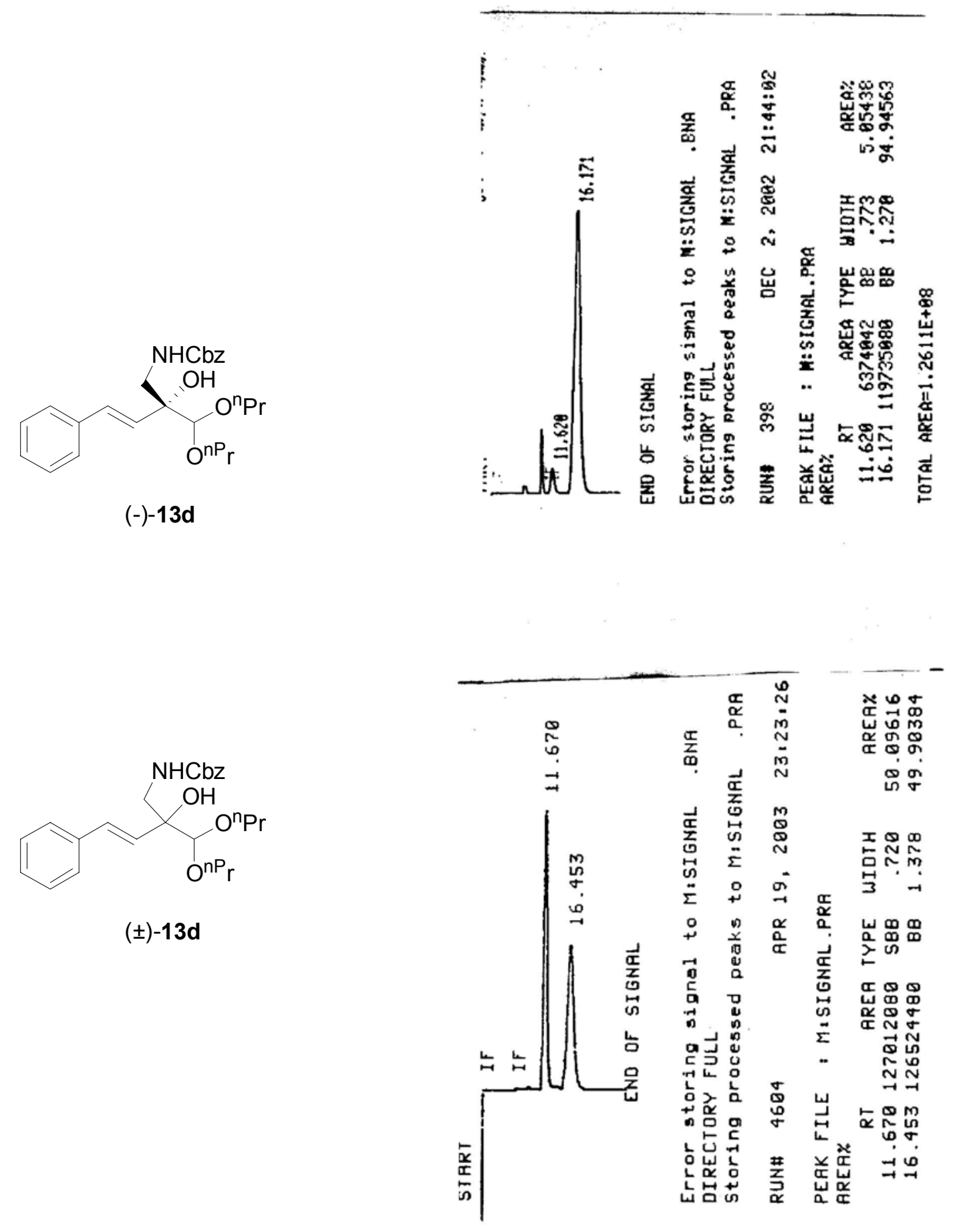
HPLC conditions: Daicel Chiralcel OD, isopropanol:hexane $/ 10: 90,1.0 \mathrm{~mL} / \mathrm{min}, \lambda=254$ $\mathrm{nm}$

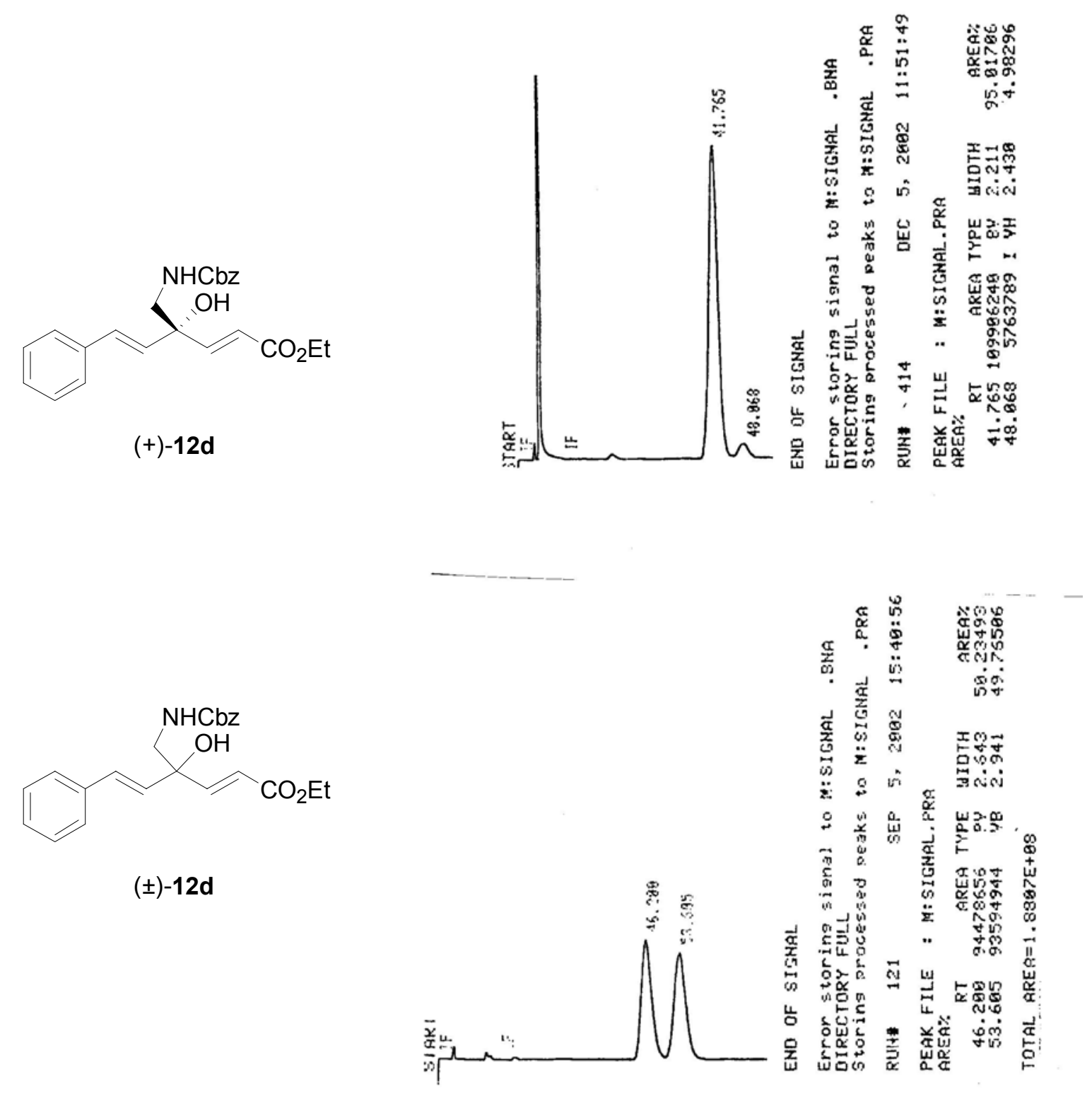

